\title{
Sorghum pan-genome explores the functional utility to accelerate the genetic gain
}

Pradeep Ruperao1, Nepolean Thirunavukkarasu², Prasad Gandham1, S. Sivasubramani ${ }^{1}$, Govindaraj M ${ }^{1}$, Baloua Nebie ${ }^{3}$, Eric Manyasa ${ }^{4}$, Rajeev Gupta ${ }^{1}$, Roma Rani Das ${ }^{1}$, Harish Gandhi ${ }^{1}$, David Edwards ${ }^{5}$, Santosh P. Deshpande ${ }^{*}$, Abhishek Rathore ${ }^{1^{*}}$

1 International Crops Research Institute for the Semi-Arid Tropics (ICRISAT), Patancheru- 502 324, Hyderabad, India

2 Genomics and Molecular Breeding Lab, ICAR-Indian Institute of Millets Research, Hyderabad-500 030, India

3 Sorghum Breeding Program, International Crops Research Institute for the SemiArid Tropics (ICRISAT), Bamako, Samanko, Mali

4 Sorghum Breeding Program, International Crops Research Institute for the SemiArid Tropics (ICRISAT), Nairobi, Kenya

5 School of Biological Sciences and Institute of Agriculture, The University of Western Australia, Perth, WA, Australia

${ }^{\star}$ Corresponding authors:

1. Dr. Abhishek Rathore

Theme Leader \& Principal Scientist (Statistics, Bioinformatics \& Data Management) International Crops Research Institute for the Semi-Arid Tropics Patancheru- 502 324

Hyderabad, Telangana, India

Email: a.rathore@cgiar.org

2. Dr. Santosh Deshpande

Senior Scientist, Research Program on Genetic Gains

International Crops Research Institute for the Semi-Arid Tropics Patancheru- 502 324 Hyderabad, Telangana, India

Email: s.deshpande@cgiar.org 


\section{Abstract}

Sorghum (Sorghum bicolor L.) is one of the most important food crops in the arid and rainfed production ecologies. It is a part of resilient farming and is projected as a smart crop to overcome the food and nutritional challenges in the developing world. The development and characterisation of the sorghum pan-genome will provide insight into genome diversity and functionality, supporting sorghum improvement. We built a sorghum pan-genome using reference genomes as well as 354 genetically diverse sorghum accessions belonging to different races. We explored the structural and functional characteristics of the pan-genome and explain its utility in supporting genetic gain. The newly-developed pan-genome has a total of 35,719 genes, a core genome of 16,821 genes and an average of 32,795 genes in each cultivar. The variable genes are enriched with environment responsive genes and classify the sorghum accessions according to their race. We show that $53 \%$ of genes display presence-absence variation, and some of these variable genes are predicted to be functionally associated with drought traits. Using more than two million SNPs from the pan-genome, association analysis identified 398 SNPs significantly associated with important agronomic traits, of which, 92 were in genes. Drought gene expression analysis identified 1,788 genes that are functionally linked to different conditions, of which 79 were absent from the reference genome assembly. This study provides comprehensive genomic diversity resources in sorghum which can be used in genome assisted crop improvement.

Keywords: Sorghum, pan-genome, diversity, SNP, gPAV, GWAS, drought genes

\section{Introduction}

Sorghum (Sorghum bicolor) is a multi-utility cereal of global importance, and a major food crop in sub-Saharan Africa and South Asia (Ritter et al., 2007),(Motlhaodi et al., $2014)$. It is typically a diploid species $(2 n=20)$ with an estimated genome size of the $\sim 800 \mathrm{Mb}$ sequence (Price et al., 2005). It provides important primary and secondary products, such as food, fodder, starch, fiber, biofuels, alcohol, dextrose syrup as well as other products. It is domesticated and further bred for diverse agro-climatic conditions ( $\mathrm{Li}$ et al., 2010) and shows wide diversity at the genome level (Hart et al., 2001; Kong et al., 2000).

A draft sorghum genome assembly of $730 \mathrm{Mb}$ was initially prepared for Sorghum bicolor Moench (Paterson et al., 2009), followed by an improved assembly of $732.2 \mathrm{Mb}$, covering approximately $91.5 \%$ of the genome (McCormick et al., 2018). Recently, a sorghum reference genome assembly for the 'Rio' line was generated comprising $729 \mathrm{Mb}$ (Cooper et al., 2019). Each of these genome assemblies is limited to its respective accession and does not reflect the diversity of genes in this species.

The presence or absence of genes or genomic regions between individuals is an important form of genomic variation in plants, and genes can be categorized into core and variable within the species (Golicz, Batley, et al., 2016; Saxena et al., 2014). The collection of these core and variable genes is known as pan-genome. A study of the pan-genome from a large number of individuals enhances the understanding of species diversity, domestication and breeding history, and provides a more complete 
characterization of species genes content diversity as demonstrated in rice (Wang et al., 2018) and tomato (Gao et al., 2019).

Several approaches are available to construct a pan-genome (Golicz, Batley, et al., 2016). The classical approach of whole-genome assembly of all genotypes was initially implemented in bacteria, and later developments led to the complementary method to "iteratively map and assemble", the unmapped sequence reads, demonstrated in B. oleracea (Golicz, Bayer, Barker, Edger, H. Kim, et al., 2016), B. napus (Hurgobin et al., 2018), bread wheat (Montenegro et al., 2017) and pigeon pea (Zhao et al., 2020). The whole genome assembly and comparison method has the advantage in that it can place almost all individual specific genes in a genomic context, but suffers from the inability to distinguish assembly or annotation errors from true biological variation (Bayer et al., 2017). It is also unsuitable for large population studies due to the expense of sequencing, assembling and comparing large numbers of genomes. In contrast, the iterative assembly approach can cost effectively assess large numbers of individuals for gene presence/absence variation and hence identify genes that may be relatively rare in a population and not samples in whole genome assembly approaches, though without additional long read data, it is unable to place many of the newly identified genes. Hence the iterative assembly method is most suited for large population diversity studies.

Hence, we assembled a pan-genome using reference and re-sequenced genomes for genetically diverse race-specific sorghum accessions. The sorghum pan-genome was initiated with the reference genome obtained from JGI on Phytozome (McCormick et al., 2018), followed by adding to this reference with novel genome sequences from 176 sorghum lines.

We provided structural and potential functional aspects of this pan-genome in the form of genes, single nucleotide polymorphism (SNP) and gene presence and absence variations (PAV). The utility of the pan-genome was demonstrated by identifying candidate functional genes using publicly available SNP chip data, genome-wide association studies and gene-expression assays. These sorghum pan-genome resources will be useful for achieving the sustainable development goals in developing countries by accelerating the genetic gain in arid and semi-arid ecologies.

\section{Results}

\section{Pan-genome assembly}

Genome sequence data with minimum 10X coverage from earlier studies (Guo et al., 2019; Valluru et al., 2019) were used for pan-genome assembly (Supplementary Table 1). The pan-genome was constructed using 176 sorghum accessions using an iterative mapping and assembly approach, similar to Brassica (Golicz, Bayer, Barker, Edger, H. Kim, et al., 2016) and pigeon pea (Zhao et al., 2020). (Figure 1). 


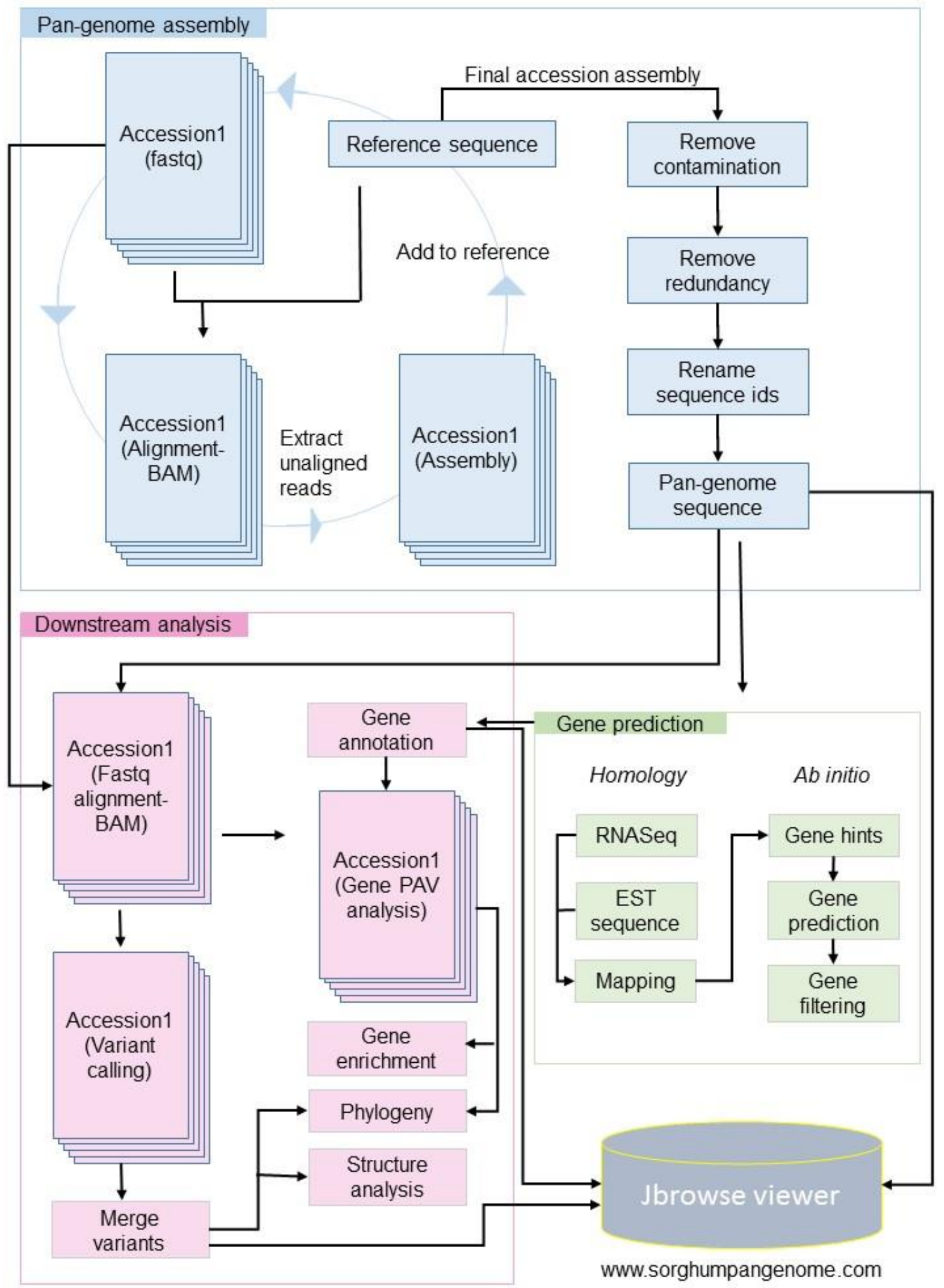

Figure 1: Raw reads of accession were aligned to reference, the unaligned reads were assembled and the contig sequence added to the reference sequence. The updated reference with assembled contigs used as reference for next iteration. The contamination and redundant sequence filtered from final updated reference and 
genes predicted with homology and ab initio method. On availability of pan-genome sequence and gene models, the downstream analysis performed including PAV, variant calling, phylogeny, enrichment and structure analysis.

On an average, each iteration of the process added $1.9 \mathrm{Mb}$ of sequence to the reference (Supplementary Figure 1) and a total of $263.7 \mathrm{Mbp}$ was assembled. Of these, $89.2 \mathrm{Mb}$ of the sequence were removed as contaminants (including chloroplast and mitochondrial sequences) and/or duplicated contigs. The final resulting pan-genome contained 210,805 contigs with a total length of $883.3 \mathrm{Mb}$ (Figure 2) with a minimum contig size of $500 \mathrm{bp}$. Gene density on the contigs added by this pan-genome exercise was lower than on assembled chromosomes but comparable to the density observed on the reference unplaced scaffolds (Figure 3). 
a

Sorghum3.0.1Ref
Length: $708,863,705$
Contigs: 870
N50: $68,658,214$
Largest Contig: $80,884,392$
Smallest Contig: 1,005

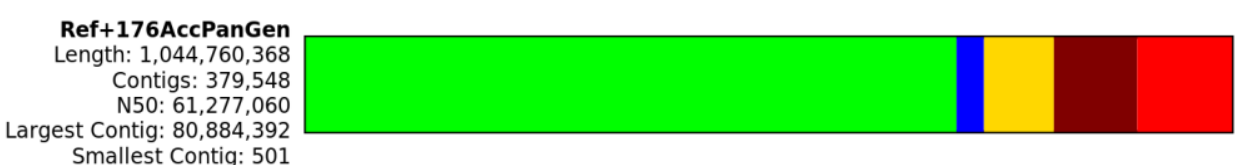

Smallest Contig: 501

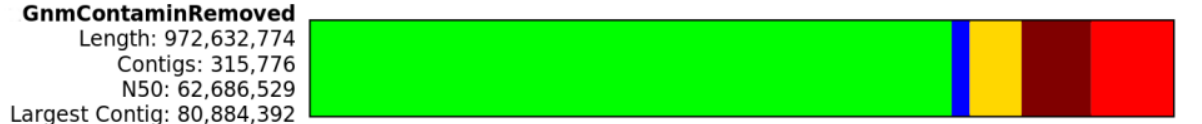

Nargest Contig: $82,6864,329$

Smallest Contig: 501

PanGnmContRmDupRm Length: $883,367,600$ Contigs: 210,805
N50: $62,686,529$

Largest Contig: 80,884,3 Smallest Contig: 50

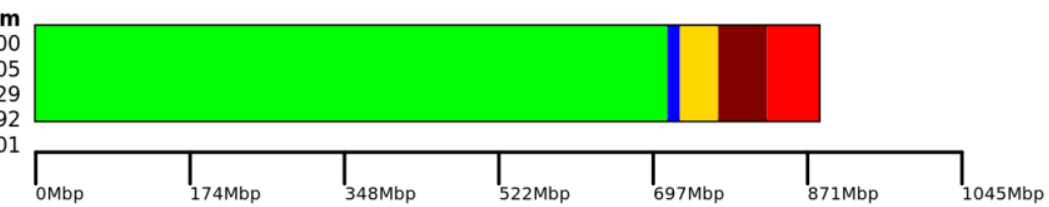

Contigs $>5 \mathrm{~kb}$

Contigs $2.5-5 \mathrm{~kb}$

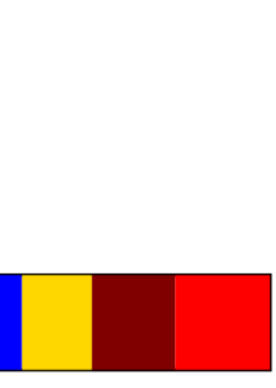

b

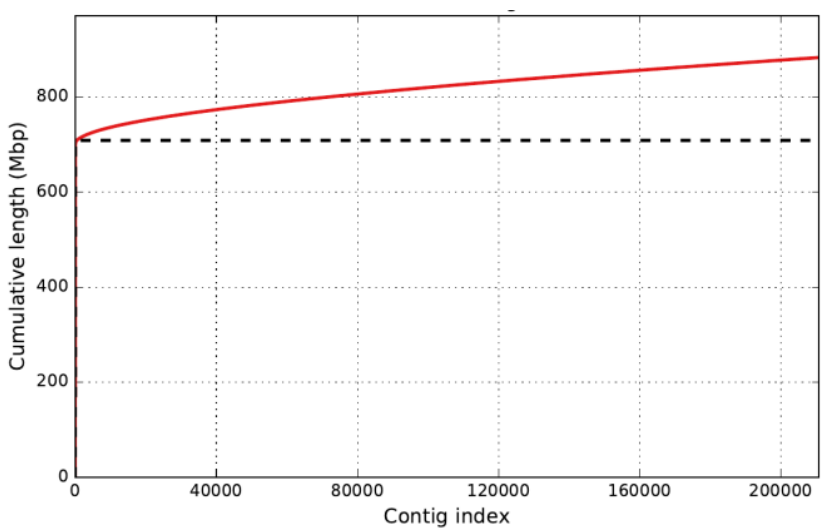

C

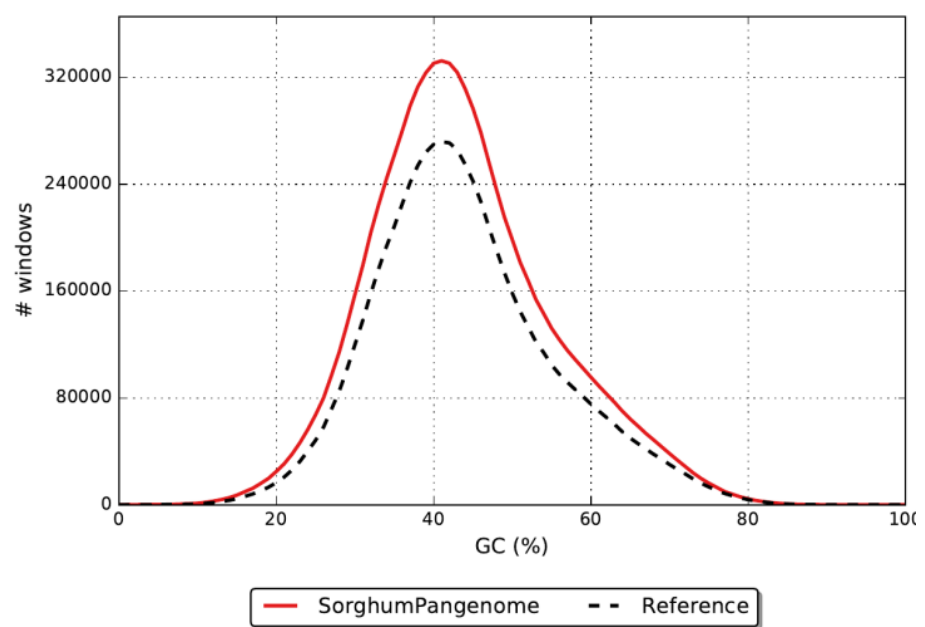

Figure 2: The development of sorghum pan-genome from reference genome assembly: a) a draft pan-genome (including sequence contamination, duplication, chloroplast and mitochondrial sequence) and final pan-genome assembly sequence size, b) cumulative length of assembled contigs and c) GC percentage of cleaned assembled contig sequences. 


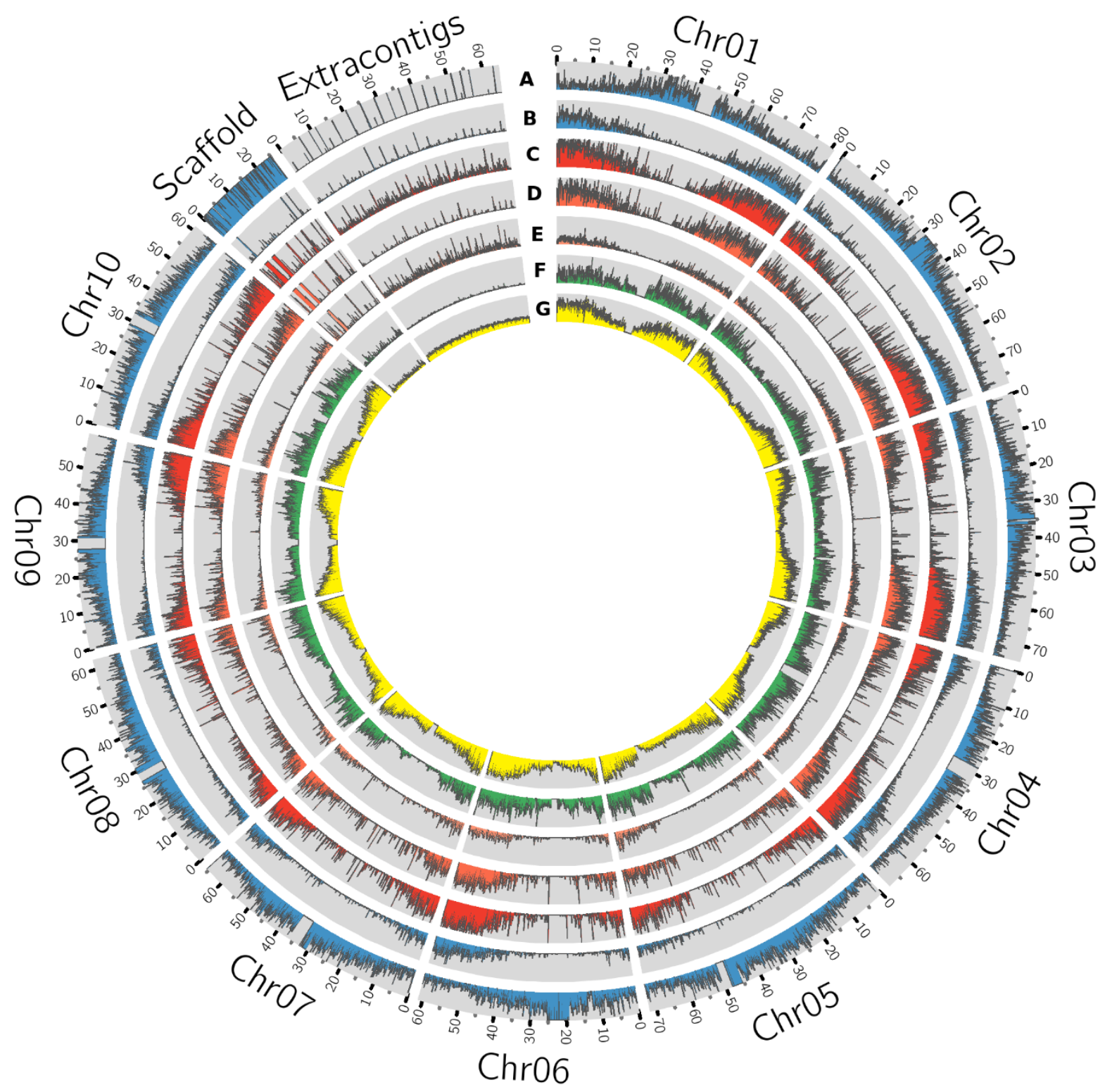

Figure 3: Circos plot of sorghum pan-genome having the extra contigs as a novel sequence assembled from 176 accessions. The genomic features of each track represent absolute values to the respective track (with $80 \mathrm{~Kb}$ window size), $\mathbf{A}$. Reference whole genome sequence reads mapping B. Drought expression (RNASeq) sequence mapping density. C. Gene density D. Genes commonly present in all accessions (core genes) E. genes absent in at least one of the accessions (variable genes) F. SNP density G. Insertions and deletions (indels).

The pan-genome showed an increase of $24.6 \%(174.5 \mathrm{Mb})$ over the reference genome (Supplementary Table 2), which was the second-biggest increase of any previously reported pan-genome after the tomato pan-genome. The increase in tomato pangenome was captured a $42 \%$ non-reference sequence from 725 accessions including the wild relatives (Gao et al., 2019). In other species, an increase in sequence size of $3.3 \%$ in wheat (Montenegro et al., 2017), 4\% in Oryza sativa japonica, 6\% in Oryza 
sativa indica (Yao et al., 2015), 5\% in Brachypodium distachyon and $20 \%$ in Brassica oleracea (Golicz, Bayer, Barker, Edger, H. Kim, et al., 2016) was documented. The relatively large increase in sorghum pan-genome assembly size indicated that the presence of high level of genome diversity in the accessions.

The assembled sequence was annotated using a strategy called combining evidencebased ab initio gene prediction. RNASeq (Guo et al., 2019) mapping hints from the 25 accessions used for ab initio gene prediction and the 3,589 genes supporting the mapped expressed sequence tags (EST) sequences were retained. We identified 11,057 to 17,616 variable genes in the 176 genomes, with an average gene sequence length and exons per gene of 1,567bp and 3.6, respectively. The gene length and exons in core genes were more than the variable genes comparatively (Figure 4). 


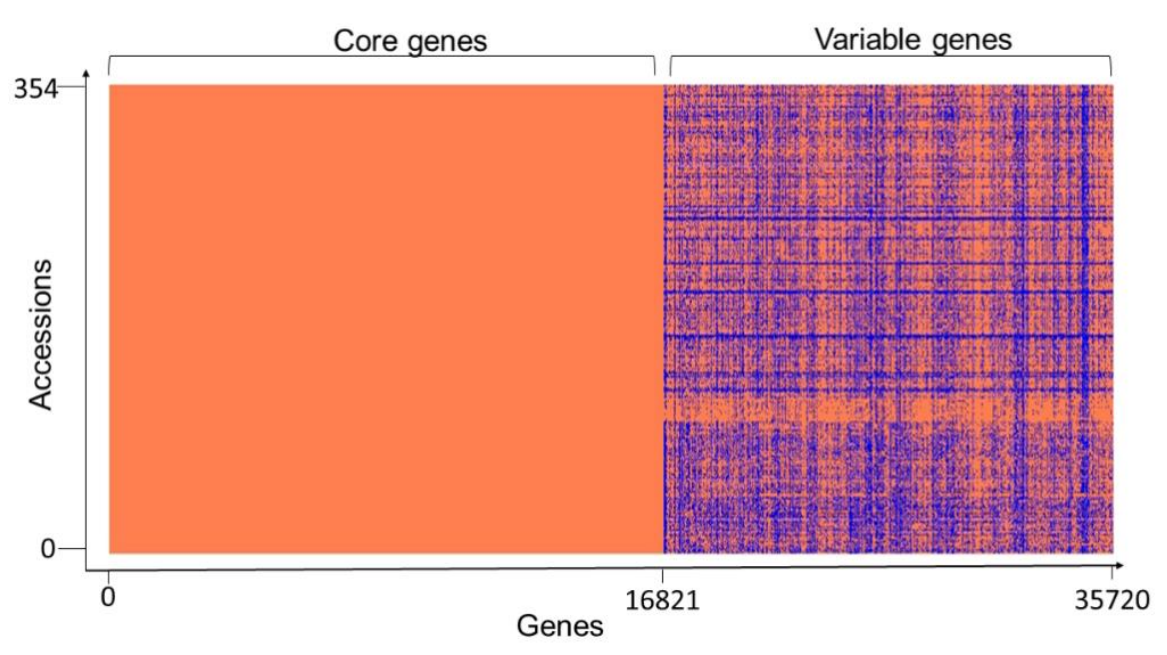

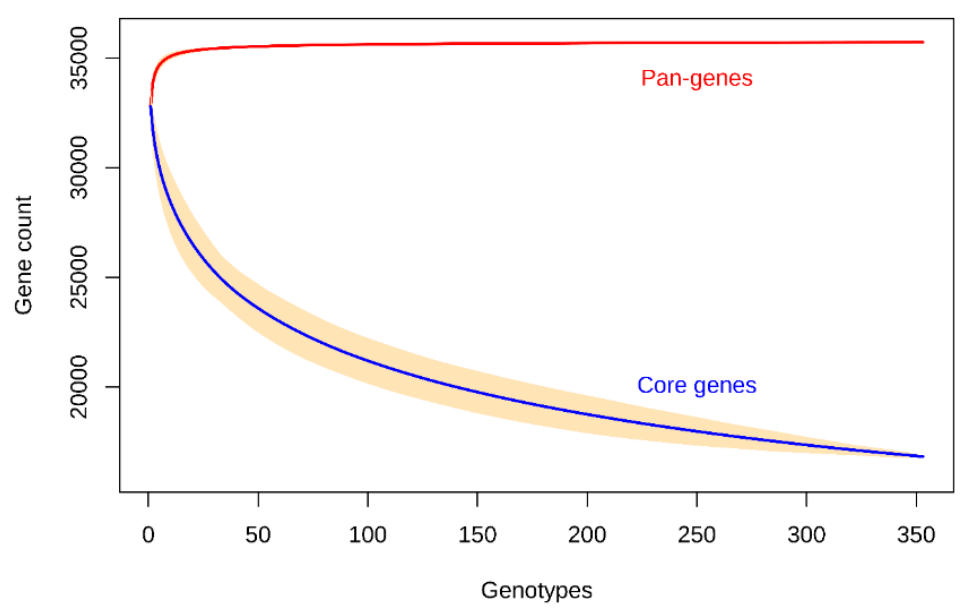

C

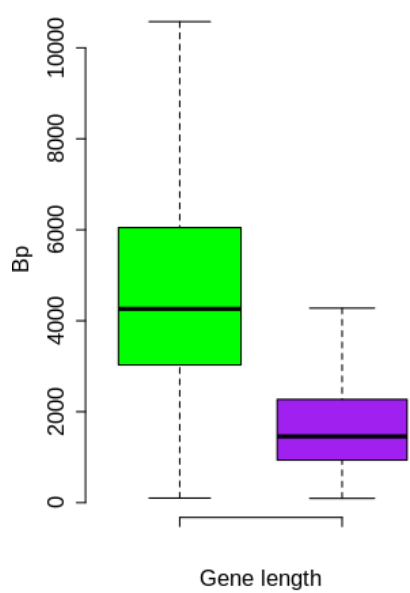

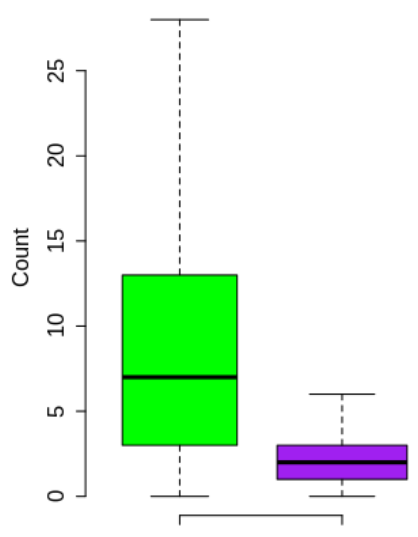

Exon number

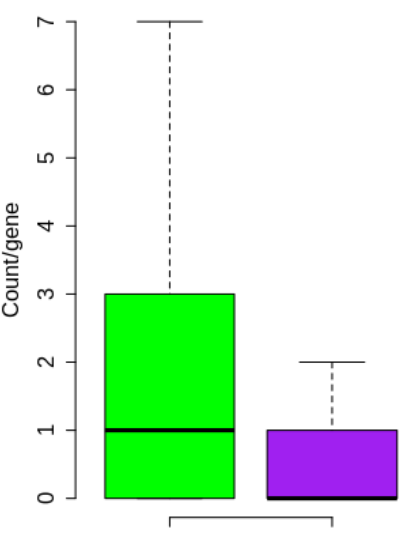

Syn SNPS

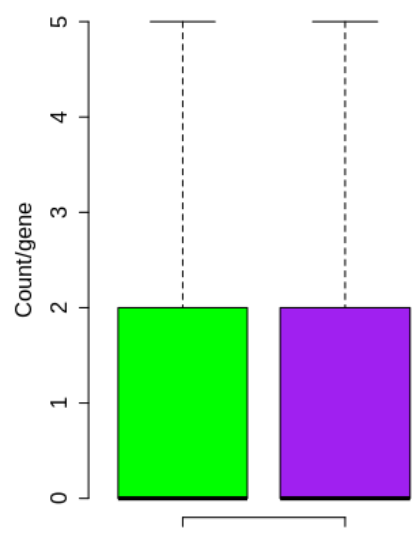

NonSyn SNPs

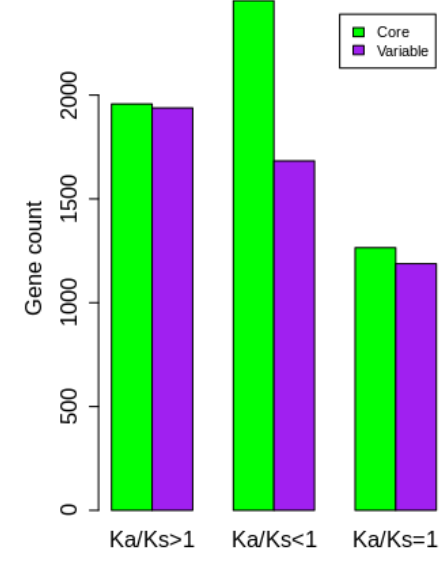

Selection

Figure 4: a) Gene presence and absence variations (gPAVs) in sorghum accessions b) Gene variation of pan-genome and core genome c) Sorghum core and variable gene properties. Variable gene length is shorter with fewer exons. The variable genes have fewer synonymous SNPs and similar non-synonymous SNPs compared to core genes. The gene counts with different $\mathrm{Ka} / \mathrm{Ks}$ category indicating the selection pressure in both core and variable genes. There is a difference in the number of SNPs between core and variable genes within all groups. 


\section{Sorghum pan-genome gene PAV (gPAV)}

The gPAV in genes among the sorghum accessions could reveal the genetic changes that can be used to infer the phylogenetic history as well as to select the potential targets for breeding. To identify the gPAVs, sequence reads were mapped to the pangenome contigs and genes were scored as present or absent based on the mapped sequence read coverage (Supplementary Table 3, Figure 5). For a given gene, to assess the gene loss event, the mapping of the whole genome sequence reads was measured. On an average, each sorghum accession contained 32,795 genes (Supplementary Table 4), of which 16,821 (47\%) were core genes or in other words, they were shared by all remaining accessions. Comparatively, tomato (Gao et al., 2019) (74.2\%), Arabidopsis thaliana (Contreras-Moreira et al., 2017) (70\%), wheat (Montenegro et al., 2017) (64\%), pigeon pea (Zhao et al., 2020) (86\%) and, Brassica napus (Hurgobin et al., 2018) (62\%) had higher number of genes (Bayer et al., 2020). On the other hand, 18,898 genes were variable/accessory genes (Figure 4.a), of which 30 genes were uniquely present and $3,183(8.9 \%)$ were uniquely absent in any one of the accessions (Supplementary Table 3, Figure 5). Variable genes were found shorter and had fewer exons per gene when compared to core genes (Figure 4.c) which were in agreement with O. sativa and A. thaliana crop studies (Bush et al., 2014; Golicz, Batley, et al., 2016; Schatz et al., 2014). Based on gPAVs from 354 cultivars, we estimated the sorghum pan-genome had a closed type of pan-genome (Figure 4.b), with 30 genes were uniquely present and 3,183 genes were uniquely absent (Supplementary Table 5). The uniquely present genes were fewer than the wheat (49 unique genes per cultivar) (Montenegro et al., 2017) and B. oleracea (37 unique genes per cultivar) (Golicz, Bayer, Barker, Edger, H. Kim, et al., 2016). Of the 30 genes uniquely present in any single sorghum accession, nine such genes were reported from Macia accession alone (Figure 5). Extending the population size and including the wild relatives could further increase the measure of the gene content of this species (Figure 4.b) (Golicz, Batley, et al., 2016). 


\section{HistogramBars}

Variable genes (min 11057, $\max 17616)$

Uniquely present genes $(\min 1, \max 9)$

Uniquely absent genes $(\min 1, \max 609)$



Figure 5: gPAVs-based neighbour-joining tree shows the genetic relationship among the sorghum accessions. Histogram shows the variable genes (purple bars), uniquely present genes (green bars) and uniquely absent genes (blue bars). 


\section{Gene functional analysis}

We identified enriched biological pathways by performing gene enrichment analysis using the $\mathrm{R}$ topGO package. The significantly enriched pathways related to responsive genes were identified (Figure 6). A total of 94 most significantly enriched genes (Supplementary Table 6) for biological process pathways are shown in Supplementary Figure 2. The gene ontology (GO) enrichment analysis showed that the genes were enriched in response to chemical, hormone, organic substance, stress, auxin and abiotic stimulus (Figure 6). It was noted that most of the pathways were related to plant response to stimulus and chemicals. The gene enrichment among stress responses genes including water deprivation (GO:0009414), desiccation (GO:0009269), abiotic stimulus (GO:0009628), chemical stimulus (G0:0042221) and stress (GO:0006950) were reported in a reference set of genes (Woldesemayat and Ntwasa, 2018). The gPAV-based enrichment on assembled genes from the sorghum pan-genome has added the response of the genes to auxin (GO:0009733), hormone (GO:0009725), organic substance (GO:0010033), hypoxia (GO:0001666) and decreased oxygen levels (GO:0036294). The functional annotation of the variable genes highlighted the genes involved in response to biotic and abiotic stress indicating the possible evolutionary adaptive traits (Lasky et al., 2015). Macia (9 genes), Ajabsido (4 genes) and PI329719 (4 genes) had a large number of unique genes (Figure 5), which could be used as potential donors for trait improvement. The functional analysis of such unique genes involved in response to the stimulus (GO:0050896), chemical (g8132, GO:0042221) and arsenic-containing substance (g24192, GO:0046685) (Supplementary Table 7). 
bioRxiv preprint doi: https:/doi.org/10.1101/2021.02.02.429137; this version posted February 3, 2021. The copyright holder for this preprint (which was not certified by peer review) is the author/funder, who has granted bioRxiv a license to display the preprint in perpetuity. It is made available under aCC-BY-NC-ND 4.0 International license.

a

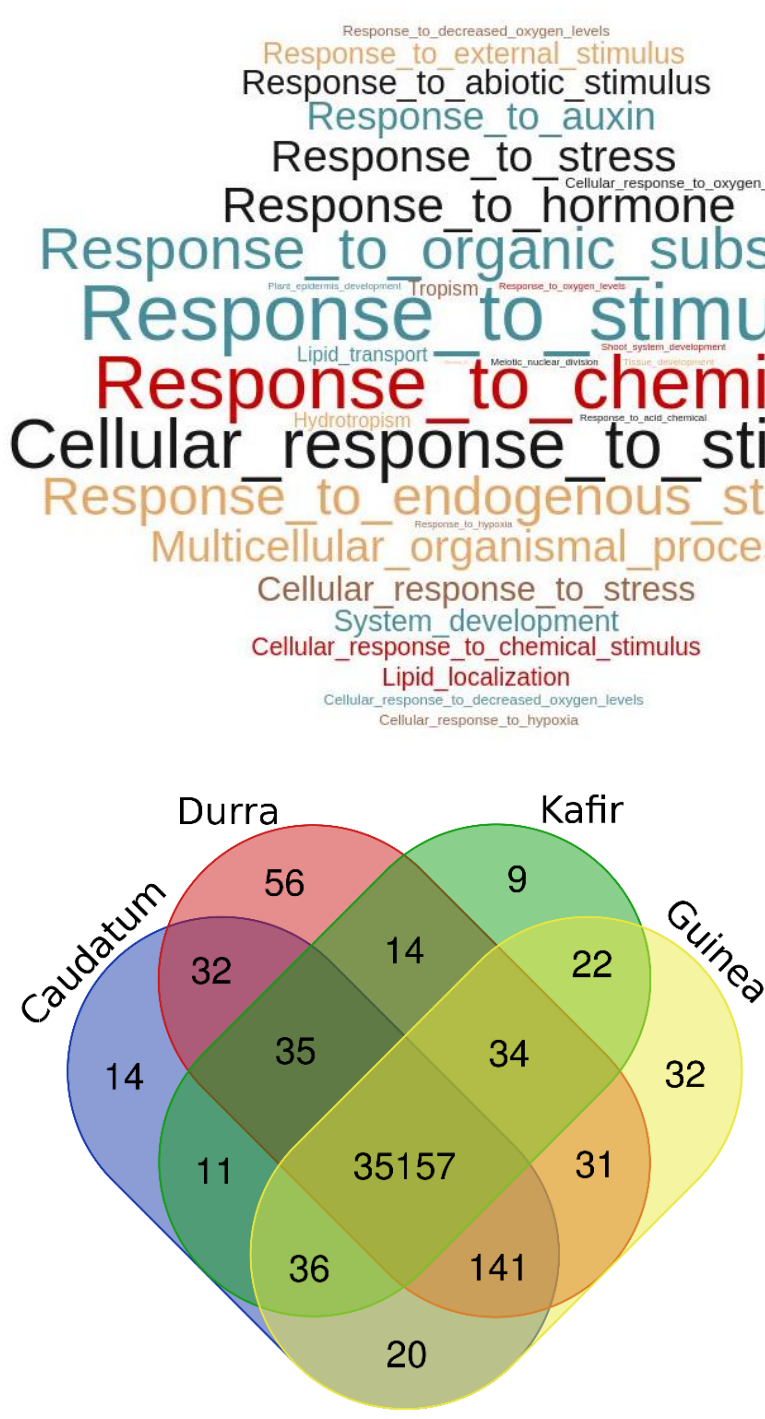

e

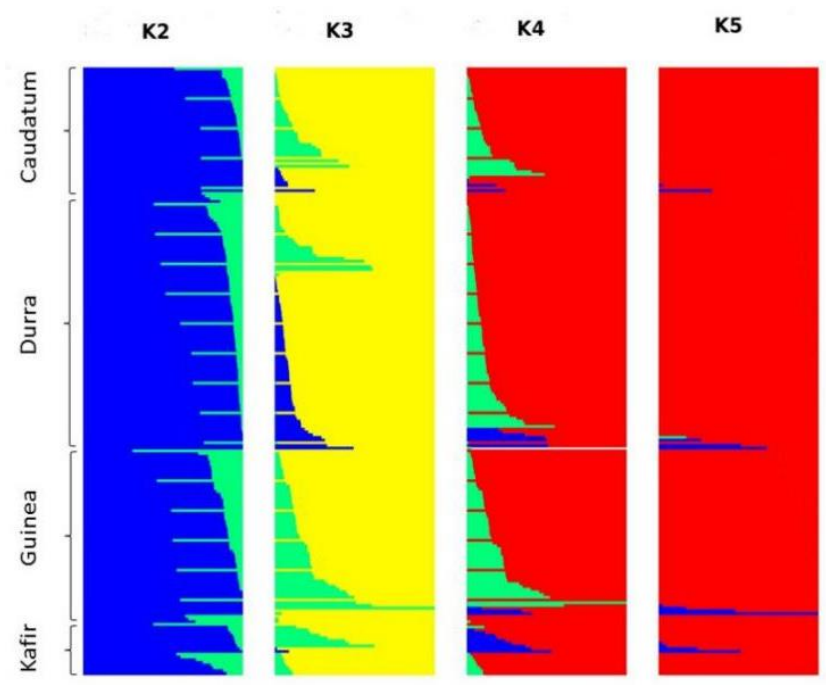

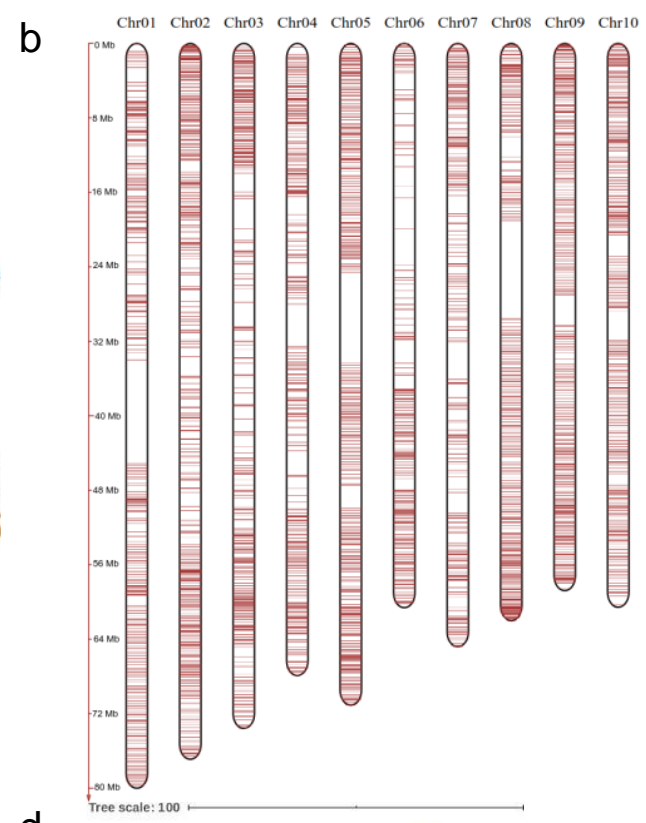

d

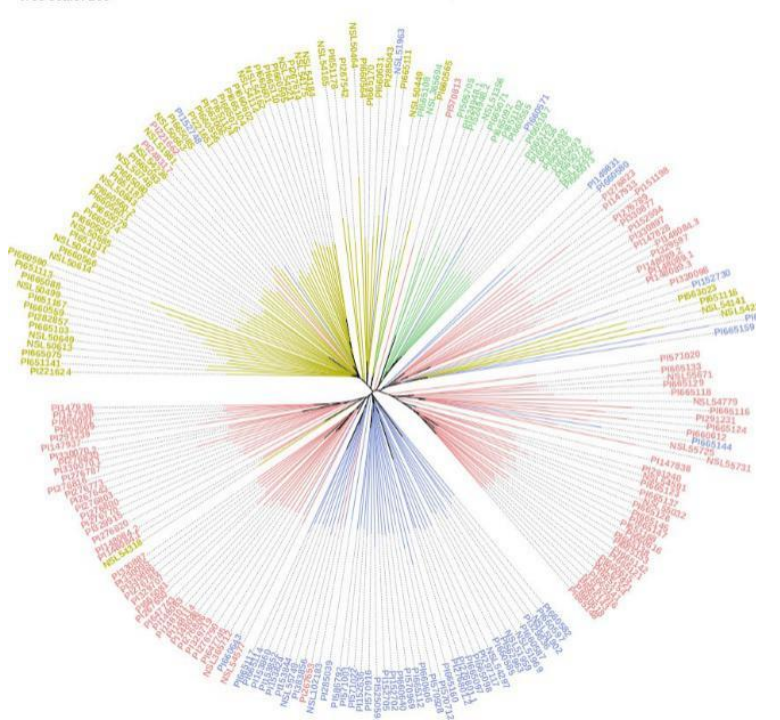

f

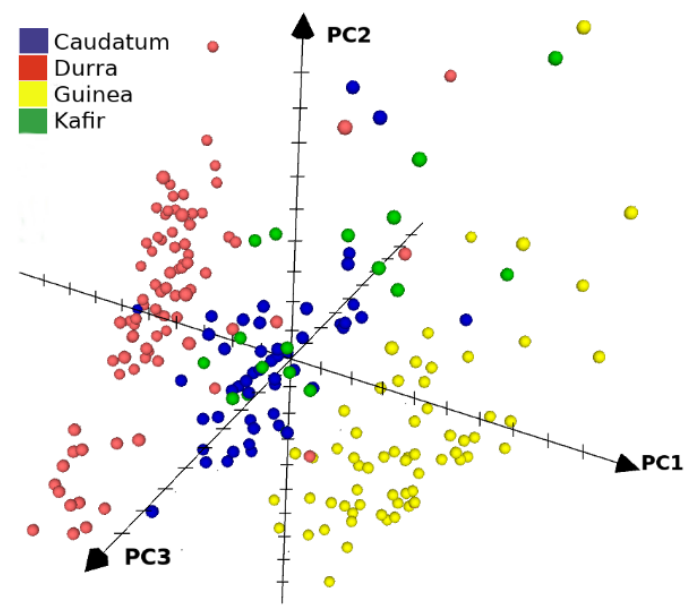

Figure 6: a) Significantly enriched top GO terms among the variable genes $(p<0.05)$ b) distribution of Infinium SNP array markers on chromosomes c) specific and common genes across races d) neighbour-joining tree shows the genetic relation among the sorghum accessions belonged to different races (blue-Caudatum, red-Durra, green-Kafir and yellow-Guinea) e) structure analysis of sorghum population individuals with $\mathrm{K} 2$ to $\mathrm{K} 5$ and f) sorghum accessions grouped by caudatum, durra, guinea and kafir race through principal co-ordinate analysis (PCo). 


\section{Variant discovery}

We identified a large number of variants (single nucleotide polymorphism (SNPS) and indels) by mapping the sorghum population whole-genome sequence reads to sorghum pan-genome assembly using GATK. Of the total of 2.0 million SNPs, 91,319 were in the extra contig (assembly) sequence (Supplementary Table 8). The SNP density in extra contigs $(0.52 / \mathrm{Kbp})$ was much less compared to the density in the reference genome assembly $(2.72 / \mathrm{Kbp})$ (Figure 3$)$. The SNP annotation results illustrated the highest number of SNPs in intergenic region $(40 \%)$ followed by upstream (22.5\%), downstream (21.4\%), intron (8.8\%) and exon (3.6\%) regions with an overall Ts/Tv ratio of 1.92 . Chromosome 4 had the highest number of SNPs $(251,830)$, followed by chromosomes $1,2,3,5,10,8,6,9$ and 7 , respectively. Chromosome 7 had the fewest number of SNPs $(119,019)$ with the highest density of $0.55 / \mathrm{Kbp}$ and chromosome 4 had the least density of $0.27 / \mathrm{Kbp}$ (Supplementary Table 8) (Figure 3). The SNPs and indels were highly dense in the telomere regions compared to centromeres explained the higher gene activity towards the telomeres. (Figure 3). The SNP annotation reported the frequency of synonymous SNPs in the core genes was much higher than in the variable genes (Figure 2). This was in contrast to the higher mis-sense SNPs in core pigeon pea genes to variable genes early reported (Zhao et al., 2020). We detected genome-wide indels of various size (Supplementary Figure 3.a) and the genes featuring indels has reduced proportionally to the size of the indels (Supplementary Figure 3.b). On increasing the indel size, the number of the indels decreases in both gene and genome-wide sequence. The overall indels count from the sorghum population used in this study was much higher than the indels earlier reported in the six sorghum lines (Yan et al., 2018). A total of 36,097 genes had 983,060 CNVs among the sorghum accessions used in this study. The $\mathrm{Ka} / \mathrm{Ks}$ ratio estimating the balance between neutral mutations, purifying selection, and beneficial mutations on a set of core and variable genes exhibited that, core gene count under positive selection were significantly close to variable gene count compared to genes under the negative selection pressure (Figure 4).

The maximum $(432,286)$ and minimum $(2,854)$ number of SNPs were identified in sorghum accessions PI267614- NSL54318 and IS3693- IS23514, respectively (Supplementary Table 9). The accessions NSL54318 $(849,052)$ and IS3693 $(17,084)$ had the maximum and minimum polymorphism, respectively with sorghum pangenome assembly sequence (Supplementary Table 10). The SNPs were validated with 3K SNPs Infinium array (Bekele et al., 2013) and of the 2,980 mapped flanking SNPs sequence, only 20 did not map on pan-genome assembly. The overall alignment rate was $99.33 \%$, from the mapped 2,980 SNPs array sequences (Supplementary Table 11, Figure 6.b). Among them, 37 SNP sequences were mapped to extra contigs (novel sequence assembly) and 150 (5\%) did not represent any GATK SNPs calls (29 SNPs from extra-contigs). In addition to the core SNPs of the array sequence, more SNPs were identified in the flanking sequence. Out of 15,383 GATK SNPs on the mapped array sequence, 15,314 SNPs were validated with the GATK called allele (Supplementary Table 11). Finally, on validation with array SNPs, the overall GATK SNP calling reported $99.9 \%$ accuracy. 
To understand the genetic relationship of the 354 sorghum accessions, a neighbour joining (NJ) tree was constructed with the SNPs (Supplementary Figure 4). The accessions were arranged in many sub-groups indicating the possible sorghum race accessions. To assess the race-specific accessions, the 216 known sorghum race accession bootstrapped to construct an $\mathrm{NJ}$ tree. The $\mathrm{NJ}$ tree showed the subgroups of sorghum accessions according to the races with an exception of few individuals placed in other groups indicating the hybridisation process in the past (Figure 6.d). For example, $\mathrm{PI} 221662$, a durra race accession was genetically related to the guinea race. Similarly, to understand the gene PAV-based genetic relation, the phylogenetic relationship among sorghum accessions was assessed by distance-based 1000 bootstrap replicates and represented through the NJ tree. Among the 35,719 total genes, $53 \%$ exhibited the genic variations to estimate the relationship among the accessions (Figure 5). The largest number of genes uniquely present and absent genes was found in Macia (9 genes) and PI660645 (372 genes), which indicated the evolutionary distance from other accessions.

With the known four races (Obilana et al., 1996), the structure analysis with the variants set showed the presence of three sub-population (Figure 6.e), resulting an expected admixture between caudataum and kafir accessions which was in agreement with early study (Valluru et al., 2019) (Figure 4). The result was also validated by the PCo, where durra and guinea sorghum races displayed identifiable clusters, because of the available sequence representation through pan-genome, while caudatum and kafir individuals exhibited the admixers (Figure 6.f). The earlier principal component study shows the mixed grouping of guinea and kafir accession in the (Sapkota et al., 2020), indicating the missing sequence representation for all race in the single reference genome. The group of guinea race accessions in PCo supported the Harlan and Stemler's hypothesis, that the guinea race was probably the first diverged race than other races from early bicolor domestication events (The Races of Sorghum in Africa, 2012).

\section{Variation of sorghum race-variable genome}

Sorghum pan-genome analysis has identified 18,898 variable genes, among which 111 were race-specific variable genes in the given population. The gene cluster analysis identified 11,470 gene families, of which un-clustered genes $(6,057)$ included 556 from the non-reference genes and the remaining 5,501 were reference genes. Among these un-clustered genes, 3,195 were orthologous to Zea mays, Setaria italica, Brachypodium distachyon, and Oryza sativa and the remaining 2,862 were paralogous. The gene shares among four sorghum races showed that the durra and guinea had a maximum of 56 and 32 unique genes, respectively making them more diverse than the other two races with 14 (caudatum) and 9 (kafir) unique genes (Figure 6.c). The gene annotations suggested that the unique genes from durra were associated with heat shock protein, LRR repeat protein, L-type lactin-domain receptor, $A B C$ transporter family proteins, and Ras-related proteins. Guinea group had the unique genes associated with disease resistance protein, beta-glucosidase proteins, NRT1/PTE protein family and Alpha/beta-Hydrolases superfamily proteins (Supplementary Table 12). The gene uniqueness to specific races possibly reflected 
bioRxiv preprint doi: https://doi.org/10.1101/2021.02.02.429137; this version posted February 3,2021 . The copyright holder for this preprint (which was not certified by peer review) is the author/funder, who has granted bioRxiv a license to display the preprint in perpetuity. It is made available under aCC-BY-NC-ND 4.0 International license.

the selection of the genotypes for adapting to the respective ecological conditions (Upadhyaya et al., 2017).

\section{Genome-wide association analysis (GWAS)}

Two populations namely, Pop1 (Valluru et al., 2019) and Pop2 (Usha Kiranmayee et al., 2020) were used for GWAS to understand the functional utility of the pan-genome. Pop1 had 216 accessions with the phenotypes of dry biomass (DBM), plant height $(\mathrm{PH})$ and starch (ST) while Pop2, a stay-green fine-mapping population with 190 segregates, had green leaf area (GLA), glossy $(G L)$, plant vigour $(V)$, leaf sheath pigment (LSP), shoot fly dead hearts (SFDH), trichome low (TL) and trichome up (TU).

In Pop1, the SNPs were further filtered by accessions and on applying the SNP quality filters, which retained 1.12 million SNPs for association analysis. Pop2 having sequence data of 190 genotypes processed to map to pan-genome and 109,338 SNPs were used for GWAS.

We identified a total of 397 unique SNPs having significant association (having p-value and false discovery rate below 0.05) in both Pop1 and Pop2 traits, of which 216 SNPs were commonly mapped with multiple traits. Most of these SNPs distributed on chromosome 10 (120 SNPs) followed by chromosome 6 (69 SNPs). The reference genome alone had 385 SNPs and the rest of the SNP-trait associations located on the unmapped read sequence assembly.

For the Pop1, a total of 36 SNPs had a significant association across three traits (Supplementary Table 13). Among them, seven were located on newly assembled contigs (DBM and $\mathrm{PH}$ ), three were from unplaced reference contigs and the remaining 26 are from chromosome sequence. Among the 36 linked SNPs, 10 were genic and the remaining 26 were inter-genic regions (Supplementary Table 14). Three of the genic SNPs were associated with DBM while six were associated with $\mathrm{PH}$ and the remaining one co-mapped to both $\mathrm{DBM}$ and $\mathrm{PH}$. From the 10 associated genes, three genes (Sobic.002G022500, Sobic.003G173400 and Sobic.004G350800) were from the core gene set and the remaining belonged to variable genes.

From the Pop2, the GLA, at various stages (Supplementary Table 15), associated with 219 SNPs including 111 genic, distributed across all chromosomes including the pangenome assembly contigs (Supplementary Table 13). GL, LSP, SFDH, TL and TU traits were associated with 129 and 103 significant SNPs in Rabi (R13) and Kharif (K13) seasons, respectively (Supplementary Table 13). The majority of the SNPs were associated with chromosome 10 followed by 5 and 6 in both the seasons. Among them, a total of 96 SNPs was mapped across seasons and a total of 18 and 196 showed season-specific association in K13 and R13, respectively. Interestingly, only four genic SNPs were associated with TU in K13, whereas 63 were associated in R13 explained the season-specific gene regulations. Similarly, SFDH had no association in K13 but had 56 genic SNPs in R13 season (Supplementary Table 15).

The number of SNPs associated with DBM, PH, ST (Pop1), plant vigour (V), GL, LSP, SFDH, TL and TU, and GLA (Pop2) was 10, 25, 1, 1, 23, 31, 84, 169, 98 and 397, respectively. Among the chromosomes, as many as 392 of the SNPs were associated with chromosome 10 and only 8 SNPs were associated with chromosome 3 (3 SNPs 
on scaffolds). The pan-genome assembly contigs hold 15 trait-associated SNPs, an additional genetic resource for the sorghum breeding program.

Of the total 183 GWAS SNPs directly associated with gene functions, the DBM and $\mathrm{PH}$ (from Pop1) were associated with 10 genes (off these, 1 gene assembled in this study). In Pop2, 173 genes were distributed as 96, 11, 13, 46, 48 and 1 for GLA, GL, LSP, SFDH, TL and TU, respectively.

a
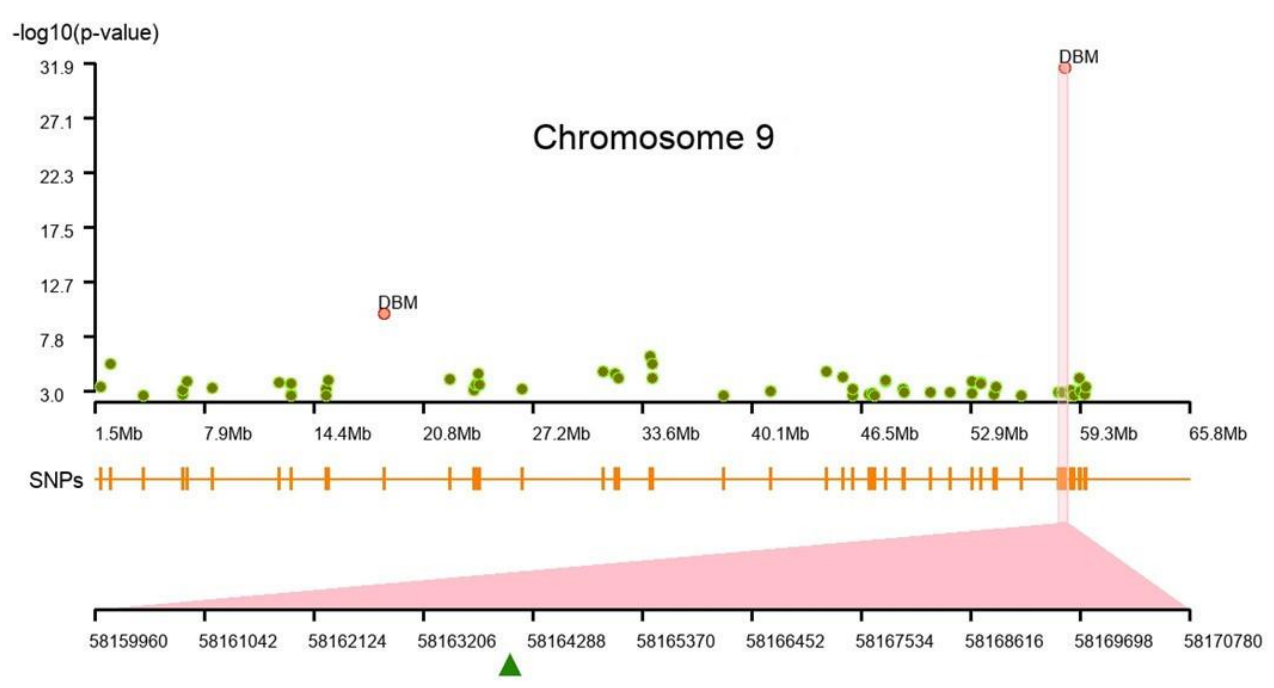

Genes $=$ Sobic.009G245900.v3.1

Sobic.009G246000.v3.1

b
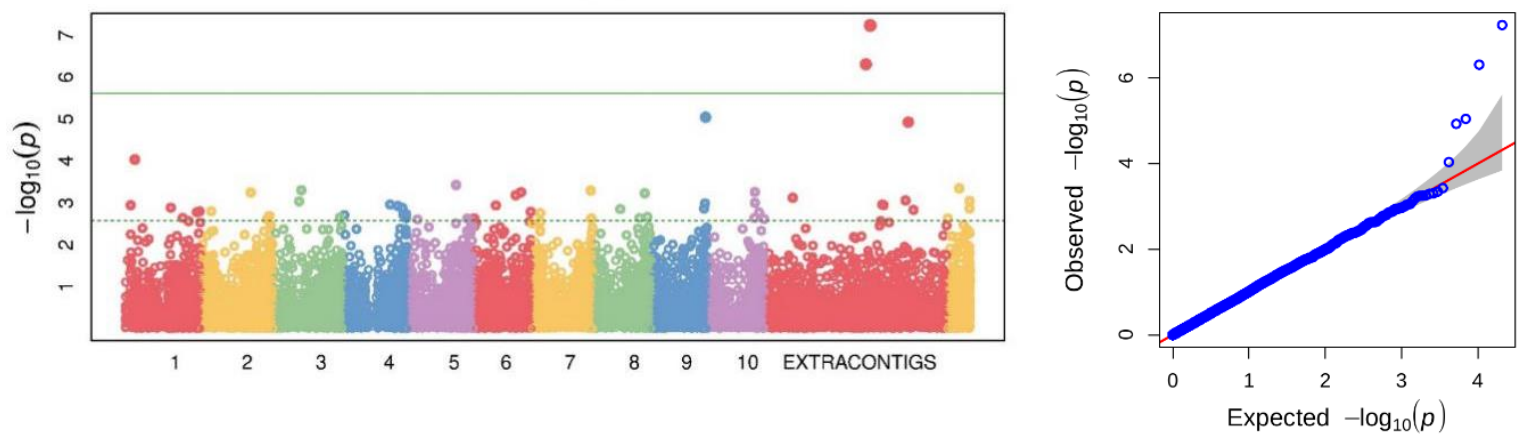

Figure 7: Genome-wide association showing the significant association of SNPs for a) plant biomass on chromosome 9 (on Sorbic.009G246000.v3.1) and b) plant height trait association on extra-contigs. 


\section{Identification of the drought candidate genes}

A sorghum RNASeq data generated from drought-resistant (BTx623 (DR1) \& SC56 (DR2)) and susceptible (Tx7000 (DS1) and PI482662 (DS2)) genotypes at different seeding stages (Abdel-Ghany et al., 2020) were re-analysed and mapped through the newly developed pan-genome. A total of 1,788 genes were significantly affected by drought stress (Figure 3 ) and among them, 79 genes were reported from genes on assembly sequence (extra- contig) (Supplementary Table 17).

The drought-resistance (DR1 and DR2) and drought-susceptibility (DS1 \& DS2) samples were phenotyped at two conditions (1 hr and 6hrs). The DR1 and DR2 samples reported ( $1 \mathrm{hrs} \mathrm{treatment)} \mathrm{a} \mathrm{total} \mathrm{of} 590$ (450 up and 140 down-regulated) and 195 (180 up- and 15 down-regulated) expressed genes respectively. Of these, none of the genes reported from the novel sequences, indicating both (DR1 and DR2) were closely related. Additionally, DR1 and the reference sequence belong to the same genotype and this supports the absence of gene expression from the novel sequence at this condition. When the treatment was extended for 6hrs, 14 (13 up and 1 downregulated) and 34 (31 up and 3 down-regulated) genes from novel sequence were expressed for DR1 and DR2 data-sets respectively.

Similarly, DS1 and DS2 samples showed (for 1 hrs treatment) 235 (123 up and 112 down-regulated) and 430 (388 up and 42 down-regulated) genes, respectively. Of these, DS1 and DS2 had 32 (1 up and 31 down-regulated) and 13 (7 up and 6 downregulated) expressed genes from novel sequence, respectively. After $6 \mathrm{hrs}$ of treatment, DS1 and DS2 samples reported 270 and 449 expressed genes respectively. Of these, DS1 and DS2 reported 8 (2 up and 6 down-regulated) and 17 (5 up and 12 down-regulated) expressed genes were from the novel sequence, respectively.

Over-all, five drought-related genes were co-mapped with the trait-associated genes. Among the five genes, three traits-linked genes Sobic.001G363200 (GLA), Sobic.007G180300 (GL) and Sobic.010G231900 (TL, TU and SFDH) were commonly expressed in drought resistance and susceptibility conditions. The remaining two drought resistance specific genes Sobic.005G069800 and Sobic.006G127800 were linked to $\mathrm{PH}$ and LSP traits. 
a
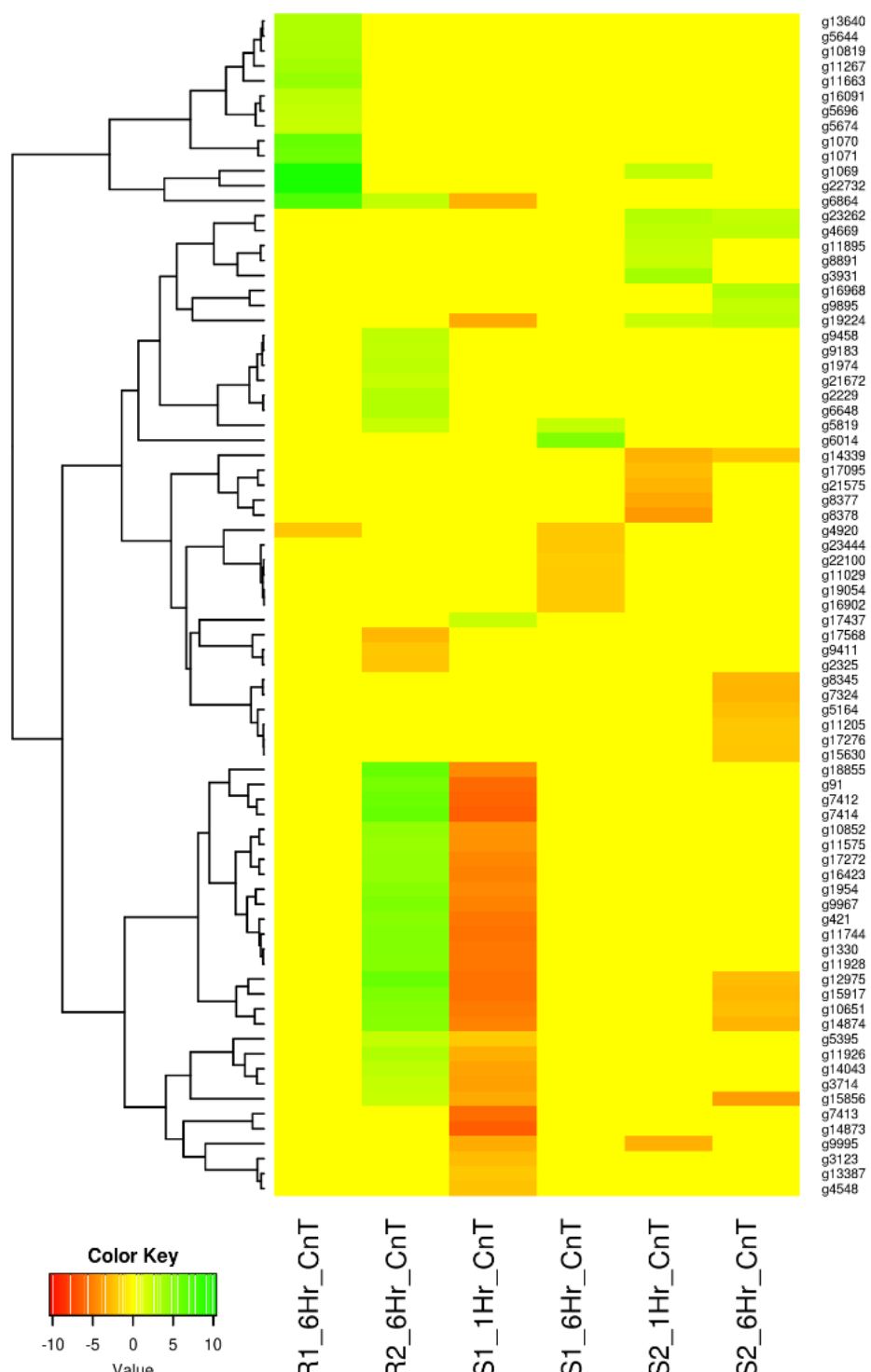

b

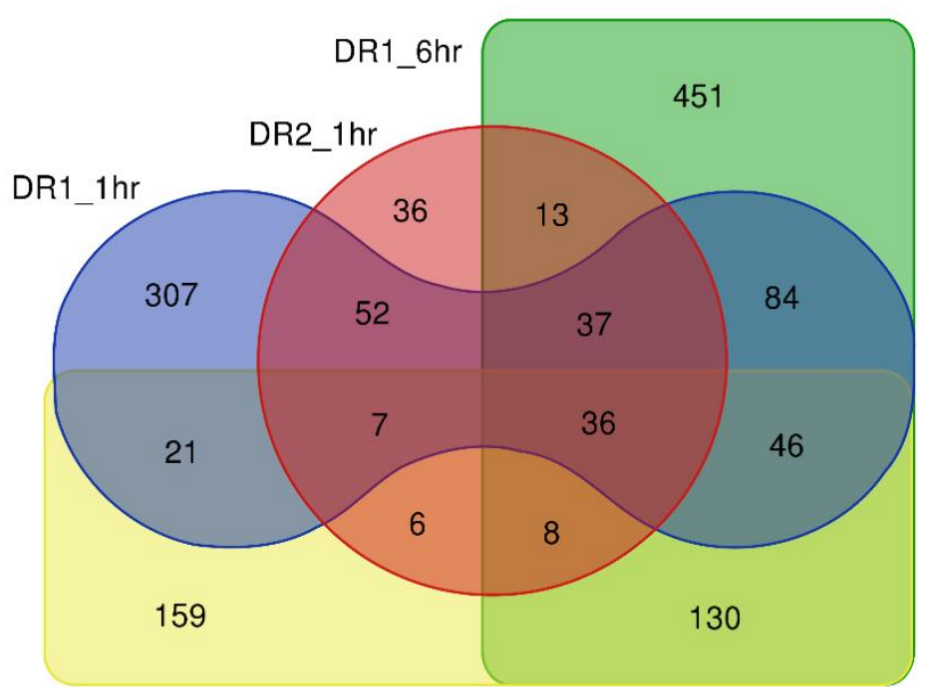

DR2_6hr

C

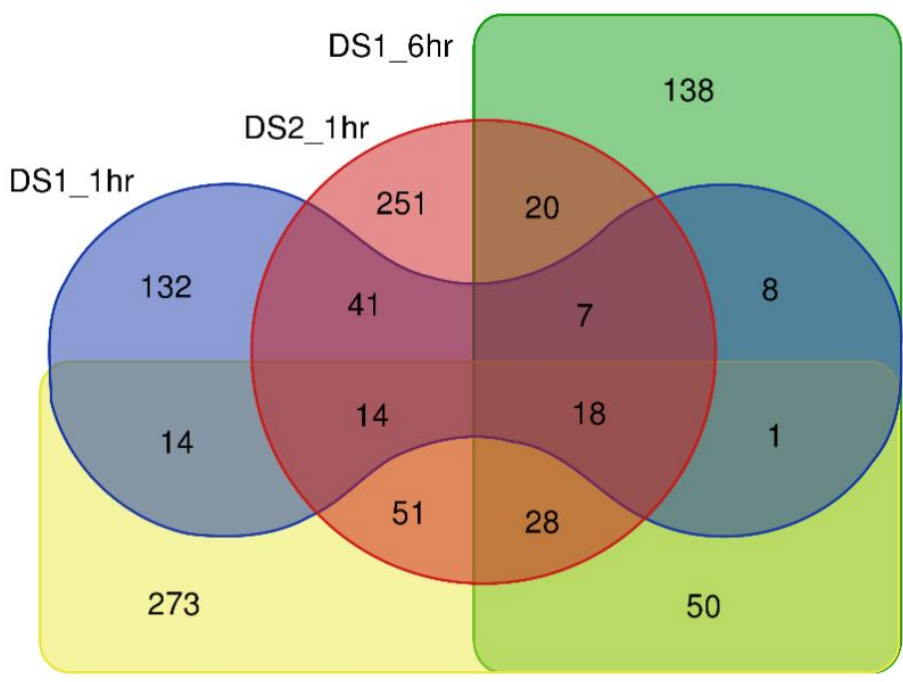

DS2_6hr

Figure 8: a) Sorghum drought trait-based RNASeq assay on pan-genome assembled genes (extra contigs). DR1 and DR2 were the two datasets for drought resistance after $1 \mathrm{hr}$ and 6hrs, respectively. DS1 and DS2 related to drought susceptibility after $1 \mathrm{hr}$ and $6 \mathrm{hrs}$, respectively. Heatmap showing the range of up-regulated genes as green and down-regulated genes as red colour b) Drought resistance in BTx623 (DR1) and SC56 (DR2) and c) Tx7000 (DS1) and PI482662 (DS2) drought susceptible genes share between the genotype and treated conditions (1hr and 6hrs). 


\section{Discussion}

We built a sorghum pan-genome with an iterative mapping and assembly approaches with 176 of 354 whole genomes sequenced accessions having coverage of more than $10 \mathrm{X}$. The total size of the pan-genome has become $883 \mathrm{Mbp}$, with a $20 \%$ increase $(175 \mathrm{Mbp})$ compare to the reference assembly of $708 \mathrm{Mbp}$. This level of novel sequence increase probably due to the high level of genetic diversity observed in the respective species (Cuevas and Prom, 2020).

We have generated the pan-genome genomic resource from the diverse sorghum accessions including the basic and intermediate sorghum races (bicolor (B), caudatum $(C)$, durra $(D)$, kafir $(K)$ and guinea $(G))$. Comparison of the wide range of sorghum whole genome sequence datasets has enabled to assemble many coding genes that were absent in published sorghum reference genome sequences. The mapping of RNASeq read from 25 accessions on the assembled contigs supports the predicted genes on the novel sequence (Supplementary Figure 5) is an additional genetic resource that will enhance the identification of the QTL and genome-wide association studies (Chen et al., 2014),(Yano et al., 2016),(Zhao et al., 2020). The earlier pangenome studies found that non-reference genes have significantly involved in agronomic traits mainly in plant defence responses (Dolatabadian et al., 2020; Golicz, Bayer, Barker, Edger, H. Kim, et al., 2016; Hirsch et al., 2014; Montenegro et al., 2017). Similar to the sorghum genes, B. oleracea pan-genome genes also showed that nearly 30 percent of reference genes exhibited the gPAV (Golicz, Bayer, Barker, Edger, H. Kim, et al., 2016). It is understood that, as the number of genotypes increases, the size of core genes decreases with a relative increase of variable genes (Figure 4.b). With the 10 sample sizes, Brassica oleracea pan-genome had $20 \%$ of PAV genes (Golicz, Bayer, Barker, Edger, H. Kim, et al., 2016) which was in consistent with simulation with similar population size in $B$. distachyon pan-genome (Gordon et al., 2017). Similarly, pan-genome from 15 Medicago genomes had $42 \%$ of sequences share with few accessions (Zhou et al., 2017), which was comparatively similar to the $49 \%$ of sorghum variable pan-genes in this study.

The result of the structure groupings correlated with the PCo showing three different clusters with one of them having two groups (caudatum and kafir). Among the four basic sorghum races used in this study, PCo displayed, guinea and durra remain as distinct clusters while caudatum with kafir classified with mixed genotypes, which is considered as the stable hybrid race among the 10 possible stable combinations of sorghum races (Obilana et al., 1996). Similarly, mixed PCo clusters were also reported earlier with five basic sorghum races, where the sorghum B race was not well supported genetically and a majority of them share membership with the remaining four genetic groups (Brown et al., 2011).

The genomic features helped the races to group into different clusters. By looking at the race-specific genomic data, each race had distinctive features. The guinea group had 37 accessions with race specific genes present in range of 2 to 13 genes per accessions, whereas durra had 2 to 12 genes in 92 accessions, kafir had 2 to 5 genes in 12 accession and caudatum had 2 to 4 genes in 15 accessions. The two groups including durra and guinea were having 56 and 32 distinct genes, respectively unique 
bioRxiv preprint doi: https://doi.org/10.1101/2021.02.02.429137; this version posted February 3, 2021. The copyright holder for this preprint (which was not certified by peer review) is the author/funder, who has granted bioRxiv a license to display the preprint in perpetuity. It is made available under aCC-BY-NC-ND 4.0 International license.

to these groups, whereas caudatum and kafir have on 14 and 9 distinct unique genes, as these groups have the admixture accessions which share genes between the groups.

The functional analysis of variable genes was enriched with GO terms associated with response to light, flower development, salt stress, water, heat, desiccation, temperature, osmotic stress, lipid, gibberellin, and stress. The results supports the earlier gene function based clustering and enrichment analysis exhibiting the similar stress respons genes reported in sorghum (Woldesemayat and Ntwasa, 2018). The plant hypersensitive response annotation in the variable gene was reported in plant pan-genome analysis (Golicz, Bayer, Barker, Edger, H. R. Kim, et al., 2016; Hurgobin et al., 2018; Montenegro et al., 2017; Zhao et al., 2020).

The development and application of sorghum SNPs have limited to reference genome assembly sequence used in the analysis. The 1.8 million SNP reported earlier on Rio with respect to BTx623 (Cooper et al., 2019), were limited to the single reference genome. Using the whole genome sequence data from 354 sorghum diverse accessions, we identified two million SNPs and 3.9 million indel sites, which represented the functional genome diversity. The density of genetic variation in the novel assembled sequence was low compared to the reference sequence. The reference genome carried most of the conserved essential genes, indicating that the variable sequence has low diversity (Figure 3), as reported in the six sorghum landrace individuals from common geographical regions (Yan et al., 2018). The fewer number of SNPs on variable sequence mainly contained genes involved in response to various stress (biotic and abiotic stress tolerance), this is in consistent with the SNPs from disease resistance $R$ genes differentiating sweet and grain sorghum accessions (Zheng et al., 2011). A reference sequence within the pan-genome assembly alone accounted for $95.4 \%$ of SNPs and the added assembly sequence from the sorghum population had $4.5 \%$ additional SNPs. A total of 2,980 array SNPs from (Bekele et al., 2013) were identified as similar to GATK called (reference-based variant calling) SNPs with $99.33 \%$ of true SNPs. The GATK called sorghum SNPs validation rate with array SNPs was higher (99.33\%) compared to the non-reference based variant calling methods, for example, the wheat pan-genome SNPs were called with $96.3 \%$ accuracy (Montenegro et al., 2017). The abundance of SNPs depends on factors such as mutation events and genome diversity and the SNPs identified in the variable genome can assist in characterizing novel metabolic pathways.

Phylogenetic analysis of 354 sorghum accessions using SNPs on the pan-genome demonstrated the mixed groups of diverse biomass genotypes (Valluru et al., 2019), domesticated accessions (Guo et al., 2019) and Chibas sorghum breeding program accessions (Jensen et al., 2020). gPAV-based phylogeny showed a group of 15 accessions having uniquely absent genes in a range of 2 to 509 genes from the biomass genotypes indicating the wider genetic diversity. The five Chibas sorghum breeding lines (Macia, Ajabsido, SC1345, P898012 and Grassl) had the most unique genes followed by seven domesticated lines distributed across the phylogenetic tree. On assessing the known sorghum race genotypes from (Valluru, et al. 2019) phylogeny showed a cluster for each sorghum race. Few individuals of caudatum and guinea were mixed with durra cluster indicating that these are the caudatum-durra 
bioRxiv preprint doi: https://doi.org/10.1101/2021.02.02.429137; this version posted February 3, 2021. The copyright holder for this preprint (which was not certified by peer review) is the author/funder, who has granted bioRxiv a license to display the preprint in perpetuity. It is made available under aCC-BY-NC-ND 4.0 International license.

(CD) and guinea-durra (GD) hybrid individuals. Similarly, few accessions were not placed in respective race groups, for example, $\mathrm{PI} 248317$ accession was a durra race accession placed in guinea race cluster which shared the genetic similarity with guinea race as DG hybrid individual.

The GWAS performed in the earlier study was limited to the phenotype association only with limited SNPs on the reference genome used (Kimani et al., 2020; Morris et al., 2013). The SNP calling on sorghum pan-genome has enabled to identify the variants also from non-reference sequence assembly from the genetically diverse accessions. A total of 91,339 SNPs reported from the assembled sequence were the additional markers used for GWAS. A total of 36 SNPs (from Pop1) were associated with target traits. Among them, 10, 25 and 1 were from assembly sequence (extra contigs) were associated with DBM, PH and ST, respectively. Additionally, the GLA (from Pop2) had a significant association with five SNPs on extra-contigs. The GLA phenotypes in 2013 and 2014 after 7, 14, 21, 28, 35, 42, 49 days after flowering (DAF) in rabi were associated with 219 SNPs. Most of the SNPs were linked with the GLA recorded at the early stage of 7 (linked with 150 SNPs) to 14 DAF (linked with 161 SNPs) (Supplementary Table 13). From the flowering stage to 14 days of postflowering, the GLA expression was significantly linked with 101 common SNPs (two SNPs reported from Extra-Contig101123 at 855 positions and Extra-Contig170379 at 501 base position) (Supplementary Table 13). For the phenotypes of GL, LSP, SFDH, TL and TU in both rabi and kharif seasons, a total of 147 SNPs were identified, of which 85 were co-mapped in both seasons, 44 were unique to rabiand 18 were unique to kharif. Out of total 397 associated SNPs, 12 SNPs from novel sequences having significant trait association is an additional gain from the pan-genome assembly.

Most of the associated SNPs linked to genes including NAC-domain protein controls the flowering time and stress response, BTB domain for protein-protein interaction, PSII protein complex for oxygenic photosynthesis, AAI domain protein for lipid transfer protein (LTP). The genes are transcription factors (TFs) such as nuclear TF, reverse transcriptase Ty1/Copia-type domain and BZIP. The genes also associated with ubiquitination pathway proteins such as B-box, F-box, U-box, RING-type and RINGtype E3 ubiquitin transferase protein supporting the sorghumFDB gene family classifications (Tian et al., 2016).

We found 1,788 drought-responsive genes with different seeding stage sequence data mapping on pan-genome assembly, whereas weelky sampled the growing plants and mapping the RNASeq data to reference alone reported the $44 \%$ of genes exhibiting the response to drought stress (Varoquaux et al., 2019).

This difference in drought expression was expected between the seedling samples (in 1 to 6 hrs difference) compared to root and leaf large scale sampling in 2 to 17 weeks of pre and post-flowering drought responses (Varoquaux et al., 2019). Similar drought stress gene expression changes were seen in laboratory and greenhouse studies in sorghum genotypes (Fracasso et al., 2016; Johnson et al., 2014). Identifying 79 drought-linked differentially expressed genes on assembly sequence are the additional genes added from this study (Supplementary Table 18). These additional genes through pan-genome were mainly involved in the cell membrane, catalytic 
activity, molecular function regulation, response to the stimulus, metabolic process, cellular and biological regulation.

\section{Methods}

\section{Pan-genome assembly and annotation}

The pan-genome was assembled using iterative mapping and assembly approaches. The assembly was initiated with a sorghum reference assembly v3.0.1 to map sorghum accession whole-genome sequence data iteratively. Reads from 176 sorghum accession were mapped to the sorghum reference v3.0 (McCormick et al., 2018) using Bowtie2 (Langmead and Salzberg, 2012) v2.3.4, and unmapped reads were assembled with IDBA_UD assembler (Peng et al., 2012) and the assembled contig sequence more than 500bp length was only considered and appended to reference genome sequence. The resulting final assembly sequence was compared with NCBI non-redundant nucleotide databases using BLASTn and the sequences with homology to sorghum mitochondria (NC_008360), chloroplast (MK348612) also the sequences having homology outside the green plant group Viridiplantae taxonomy group (Taxonomy ID: 33090) were removed. The remaining sequences were selfcompared with nucmer search (http://mummer.sourceforge.net/) and sequences with greater than 90 percent coverage with greater 90 percent identity were removed to maintain the non-redundancy of the novel sequences. REPEATMASKER-v4.0.7 (Smit, AFA, Hubley, R \& Green) masked repetitive elements using sorghum as the species. The sorghum expressed sequence tags (ESTs) from GenBank were aligned with tBLASTx and genes were predicted using AUGUSTUS v3.3.2, supporting the EST alignments. The gene models having fewer than $300 \mathrm{bp}$ in length were filtered out and the remaining genes supporting either EST alignments or hisat2 (Kim et al., 2019) alignments (RNASeq read from 25 accessions, Supplementary Table 1) further used for functional annotation against uniref90 (database downloaded in May 2020).

\section{Gene presence-absence variations (gPAVs)}

Whole-genome sequence reads of 354 sorghum lines were mapped with Bowtie2 v2.3.4 (Langmead and Salzberg, 2012) to pan-genome assembly with a wide insert size range between 0 and $1000 \mathrm{bp}$. The gene PAVs were defined based on sequence reads coverage mapped to respective genes as described by (Golicz, Bayer, Barker, Edger, H. Kim, et al., 2016). Genes models on contigs longer than $1 \mathrm{Kbp}$ were used in this analysis. PAV converted into binary matrix and with 1000 bootstrap resampling were used to estimate the genetic relationship among the accessions with $\mathrm{R}$ "ape" package (Paradis et al., 2004) to construct a NJ tree and visualized in iTOL tree viewer (Letunic and Bork, 2019).

The core genes were defined as the genes present in all the accessions, whereas the variable genes are the genes missing in one or more accessions. The in-house developed script was used to define the core and variable genes from the PAV matrix. Core and variable genes were compared for gene length, exon number, synonymous SNPs, non-synonymous SNPs and $\mathrm{Ka} / \mathrm{Ks}$. The mean count for each sample size of core and pan-genes present in all possible combinations of 354 accessions was plotted. The protein sequences of Zea mays, Setaria italica, Brachypodium 
distachyon, and Oryza sativa were downloaded from the public database UniProt for cluster analysis. All protein sequences were compared using all-by-all BLASTp followed by MCL for gene clustering into gene families with default parameters. The gene enrichment analysis was performed with Fisher exact test from R "topGO" package (Alexa et al., 2006) using "Elim" method.

\section{SNP discovery and annotation}

The sorghum whole genome sequence reads of 354 accessions were quality trimmed using Trimmomatic (Bolger et al., 2014) and mapped to pan-genome using Bowtie2 v2.3.4 (Langmead and Salzberg, 2012) allowing to map paired reads. The aligned reads in SAM format converted to BAM format using samtools (Li et al., 2009) followed by filtering out the read duplication with Picard tools (http://broadinstitute.github.io/picard). Variants against the reference (pan-genome) were called with GATK v.4.1 (McKenna et al., 2010) and directed to quality filtered with vcftools v.0.1.13 (Danecek et al., 2011). The variant sites having missing genotypes of more than 0.15 and minor allele count less than two were excluded and the remaining sites were used for downstream analysis such as SNP functionally annotated with SnpEff v.4.3 (Cingolani et al., 2012).

\section{Sorghum diversity and population structure}

A panel of 216 diverse sorghum accessions, including the four races, were used for genetic diversity and population structure assessment. A total of 1.12 million filtered SNPs from sorghum race accessions were retained for downstream analysis. The STRUCTURE v2.3 (Hubisz et al., 2009), was used to estimate the population structure using admixture model. The tested $\mathrm{K}$ was set from 2 to 5 and optimal $\mathrm{K}$ for population structure was defined with the structure program. With the same SNP set, PCo analysis was done with $R$ labdsv package (https://CRAN.Rproject.org/package=labdsv) and phylogeny analysis performed using 1000 replicates with R "ape" package (Paradis et al., 2004) and visualized in iTOL tree viewer (Letunic and Bork, 2019).

\section{GWAS}

Two different mapping populations having the phenotypic data of ten traits were used for the association study.

Pop1: The phenotype and genotype data associated with $\mathrm{PH}, \mathrm{DBM}$ and ST were adapted from (Valluru et al., 2019) for GWAS analysis. Among the 354 WGS data used for the above analysis, 227 genotypes belonged to four major races of sorghum having a representation from Africa, Asia and America. The SNPs corresponding to the above-mentioned genotypes were filtered with vcftools and used for GWAS. In 2016, the $\mathrm{PH}$ was recorded from 4 to 16 weeks after planting with an interval of 2 weeks, DBM and ST was measured at harvest.

Pop2: The stay-green fine-mapping population developed by crossing an introgression line cross RSG04008-6 × J2614-11 (Usha Kiranmayee et al., 2020) was used for association study using the pan-genome assembly. The DNA from parents and 152 individuals were isolated and skim-sequenced to produce genotype data to a depth of 
0.1X. The sequence reads were QC'd with trimmomatic (Bolger et al., 2014), mapped with bowtie2 (Langmead and Salzberg, 2012) and SNP called with GATK (McKenna et al., 2010) and filtered with vcftools (Danecek et al., 2011) as above said method.

The Pop2 was evaluated with GLA trait in the rabi season of 2012-2013 and 20132014 at ICRISAT, Patancheru, India. The GLA percentage was measured from seven to 49 DAF for every seven days interval in both years. Additionally, in the year 2013, the phenotypes of GL, LSP, V, TL, TU, SFDH traits were recorded in rabi (R13) and kharif (K13) seasons.

The genotype to phenotype association was performed with GAPIT (Lipka et al., 2012) and the results were initially filtered with Bonferroni cut-off $(-\log 10(p$-value $)>2.5)$ followed by $p$-value and false discovery rate values less than 0.05 (close to BenjaminiHochberg cut-off value) as the significant values. On parsing the gene coordinates, for each candidate SNP within the range of genes were identified and assigned to SNP.

\section{Drought RNASeq assay analysis}

The RNASeq data derived from two genotypes of drought-resistant (BTx623 (DR1) \& SC56 (DR2)) and drought susceptible (Tx7000 (DS1) and PI482662 (DS2)) at the seedling stage was obtained from (Abdel-Ghany et al., 2020) SRA database. The quality check was performed on raw sequence reads using FastQC (Andrews, 2015) followed by cleaning the low-quality reads and removing sequencing adaptors using the Trimmomatic (Bolger et al., 2014) tool. Trimmed reads were aligned to the Sorghum pan-genome using TopHat2 (Kim et al., 2013) and bam files were filtered to remove reads aligned to multiple locations. Differential gene expression was performed on different conditions using Cuffdiff (Trapnell et al., 2010) to compute $\log \mathrm{FC}$ and q-values across all lines at different conditions (control \& treated). A total of eight conditions were analysed to find drought-induced genes after $1 \mathrm{hr}$ and $6 \mathrm{hrs}$ of post-treatment (20\% PEG (polyethyleneglycol) treatment). Two biological replicates were analysed for each condition resulting in 32 samples (4 genotypes $\times 2$ conditions $\times 2$-time points $\times 2$ replicates). The differentially expressed genes (DEGs) were determined if the q-value is less than 0.05 and log2FC is $<-2$ or $>2$ ratios between control and treatment for each time point and in each genotype.

\section{Conclusions}

We constructed and characterised the sorghum pan-genome using the reference genome assembly and the whole-genome sequence reads of genetically diverse sorghum accessions. The pan-genome had 35,719 predicted genes, which were categorized as core, conserved genes, and variable genes as they exhibited presence and absence variation. The variable genes were enriched with genes response to various stresses. The SNP Infinium array result showed $99 \%$ of representation on the pan-genome assembly sequence. About two million SNPs were developed through pan-genome which can use for functional downstream research. The pan-genome resources were validated by assessing the genetic diversity of sorghum races, identification of genes from GWAS and RNASeq studies. These newly generated genomic resources could be used in sorghum genetic gain improvement programs. 


\section{Acknowledgments}

The authors thank Plant Breeding and Genetics Section, School of Integrative Plant Science, Cornell University, Ithaca, New York, USA and Plant Genome Mapping Laboratory, University of Georgia, Athens, Georgia, USA for providing the sorghum WGRS, RNASeq data and phenotype data. The authors also acknowledge the supporting funds from AVISA, ICAR and BMGF.

\section{Author contributions}

PR and AR conceived and designed the project. PR, PG and SS carried out the analysis. SS managed computational resources and data management. SDP provided sorghum trait data. PR, NT, DE, GM, NB, RG and AR jointly wrote the manuscript. All authors have seen and the manuscript hasn't been published elsewhere.

\section{Competing interests}

The authors declare no competing interests.

\section{Reference}

Abdel-Ghany, S.E., Ullah, F., Ben-Hur, A., and Reddy, A.S.N. (2020) Transcriptome analysis of drought-resistant and drought-sensitive sorghum (Sorghum bicolor) genotypes in response to peg-induced drought stress. Int. J. Mol. Sci.

Alexa, A., Rahnenführer, J., and Lengauer, T. (2006) Improved scoring of functional groups from gene expression data by decorrelating GO graph structure. Bioinformatics.

Andrews, S. (2015) FASTQC A Quality Control tool for High Throughput Sequence Data. Babraham Inst.

Bayer, P.E., Golicz, A.A., Scheben, A., Batley, J., and Edwards, D. (2020) Plant pangenomes are the new reference. Nat. Plants.

Bayer, P.E., Hurgobin, B., Golicz, A.A., Chan, C.K.K., Yuan, Y., Lee, H.T., et al. (2017) Assembly and comparison of two closely related Brassica napus genomes. Plant Biotechnol. J.

Bekele, W.A., Wieckhorst, S., Friedt, W., and Snowdon, R.J. (2013) High-throughput genomics in sorghum: From whole-genome resequencing to a SNP screening array. Plant Biotechnol. J.

Bolger, A.M., Lohse, M., and Usadel, B. (2014) Trimmomatic: A flexible trimmer for Illumina sequence data. Bioinformatics.

Brown, P.J., Myles, S., and Kresovich, S. (2011) Genetic support for phenotypebased racial classifi cation in sorghum. Crop Sci.

Bush, S.J., Castillo-Morales, A., Tovar-Corona, J.M., Chen, L., Kover, P.X., and Urrutia, A.O. (2014) Presence-absence variation in A. thaliana is primarily associated with genomic signatures consistent with relaxed selective constraints. Mol. Biol. Evol., 31, 59-69.

Chen, W., Gao, Y., Xie, W., Gong, L., Lu, K., Wang, W., et al. (2014) Genome-wide 
association analyses provide genetic and biochemical insights into natural variation in rice metabolism. Nat. Genet.

Cingolani, P., Platts, A., Wang, L.L., Coon, M., Nguyen, T., Wang, L., et al. (2012) $A$ program for annotating and predicting the effects of single nucleotide polymorphisms, SnpEff. Fly (Austin).

Contreras-Moreira, B., Cantalapiedra, C.P., García-Pereira, M.J., Gordon, S.P., Vogel, J.P., Igartua, E., et al. (2017) Analysis of Plant Pan-Genomes and Transcriptomes with GET_HOMOLOGUES-EST, a Clustering Solution for Sequences of the Same Species. Front. Plant Sci., 8.

Cooper, E.A., Brenton, Z.W., Flinn, B.S., Jenkins, J., Shu, S., Flowers, D., et al. (2019) A new reference genome for Sorghum bicolor reveals high levels of sequence similarity between sweet and grain genotypes: Implications for the genetics of sugar metabolism. BMC Genomics.

Cuevas, H.E. and Prom, L.K. (2020) Evaluation of genetic diversity, agronomic traits, and anthracnose resistance in the NPGS Sudan Sorghum Core collection. BMC Genomics.

Danecek, P., Auton, A., Abecasis, G., Albers, C.A., Banks, E., DePristo, M.A., et al. (2011) The variant call format and VCFtools. Bioinformatics.

Dolatabadian, A., Bayer, P.E., Tirnaz, S., Hurgobin, B., Edwards, D., and Batley, J. (2020) Characterization of disease resistance genes in the Brassica napus pangenome reveals significant structural variation. Plant Biotechnol. J.

Fracasso, A., Trindade, L.M., and Amaducci, S. (2016) Drought stress tolerance strategies revealed by RNA-Seq in two sorghum genotypes with contrasting WUE. BMC Plant Biol.

Gao, L., Gonda, I., Sun, H., Ma, Q., Bao, K., Tieman, D.M., et al. (2019) The tomato pan-genome uncovers new genes and a rare allele regulating fruit flavor. Nat. Genet.

Golicz, A.A., Batley, J., and Edwards, D. (2016) Towards plant pangenomics. Plant Biotechnol. J., 14, 1099-1105.

Golicz, A.A., Bayer, P.E., Barker, G.C., Edger, P.P., Kim, H., Martinez, P.A., et al. (2016) The pangenome of an agronomically important crop plant Brassica oleracea. Nat. Commun., 7, 13390.

Golicz, A.A., Bayer, P.E., Barker, G.C., Edger, P.P., Kim, H.R., Martinez, P.A., et al. (2016) The pangenome of an agronomically important crop plant Brassica oleracea. Nat. Commun.

Gordon, S.P., Contreras-Moreira, B., Woods, D.P., Des Marais, D.L., Burgess, D., Shu, S., et al. (2017) Extensive gene content variation in the Brachypodium distachyon pan-genome correlates with population structure. Nat. Commun.

Guo, H., Jiao, Y., Tan, X., Wang, X., Huang, X., Jin, H., and Paterson, A.H. (2019) Gene duplication and genetic innovation in cereal genomes. Genome Res.

Hart, G.E., Schertz, K.F., Peng, Y., and Syed, N.H. (2001) Genetic mapping of Sorghum bicolor (L.) Moench QTLs that control variation in tillering and other 
morphological characters. Theor. Appl. Genet.

Hirsch, C.N., Foerster, J.M., Johnson, J.M., Sekhon, R.S., Muttoni, G., Vaillancourt, B., et al. (2014) Insights into the Maize Pan-Genome and Pan-Transcriptome. Plant Cell, 26, 121-135.

Hubisz, M.J., Falush, D., Stephens, M., and Pritchard, J.K. (2009) Inferring weak population structure with the assistance of sample group information. Mol. Ecol. Resour.

Hurgobin, B., Golicz, A.A., Bayer, P.E., Chan, C.K.K., Tirnaz, S., Dolatabadian, A., et al. (2018) Homoeologous exchange is a major cause of gene presence/absence variation in the amphidiploid Brassica napus. Plant Biotechnol. J.

Jensen, S.E., Charles, J.R., Muleta, K., Bradbury, P.J., Casstevens, T., Deshpande, S.P., et al. (2020) A sorghum practical haplotype graph facilitates genome-wide imputation and cost-effective genomic prediction. Plant Genome.

Johnson, S.M., Lim, F.L., Finkler, A., Fromm, H., Slabas, A.R., and Knight, M.R. (2014) Transcriptomic analysis of Sorghum bicolor responding to combined heat and drought stress. BMC Genomics.

Kim, D., Paggi, J.M., Park, C., Bennett, C., and Salzberg, S.L. (2019) Graph-based genome alignment and genotyping with HISAT2 and HISAT-genotype. Nat. Biotechnol.

Kim, D., Pertea, G., Trapnell, C., Pimentel, H., Kelley, R., and Salzberg, S.L. (2013) TopHat2: Accurate alignment of transcriptomes in the presence of insertions, deletions and gene fusions. Genome Biol.

Kimani, W., Zhang, L.M., Wu, X.Y., Hao, H.Q., and Jing, H.C. (2020) Genome-wide association study reveals that different pathways contribute to grain quality variation in sorghum (Sorghum bicolor). BMC Genomics.

Kong, L., Dong, J., and Hart, G.E. (2000) Characteristics, linkage-map positions, and allelic differentiation of Sorghum bicolor (L.) Moench DNA simple-sequence repeats (SSRs). Theor. Appl. Genet.

Langmead, B. and Salzberg, S.L. (2012) Fast gapped-read alignment with Bowtie 2. Nat. Methods.

Lasky, J.R., Upadhyaya, H.D., Ramu, P., Deshpande, S., Hash, C.T., Bonnette, J., et al. (2015) Genome-environment associations in sorghum landraces predict adaptive traits. Sci. Adv.

Letunic, I. and Bork, P. (2019) Interactive Tree of Life (iTOL) v4: Recent updates and new developments. Nucleic Acids Res.

Li, H., Handsaker, B., Wysoker, A., Fennell, T., Ruan, J., Homer, N., et al. (2009) The Sequence Alignment/Map format and SAMtools. Bioinformatics.

Li, R., Zhang, H., Zhou, X., Guan, Y., Yao, F., Song, G., et al. (2010) Genetic diversity in Chinese sorghum landraces revealed by chloroplast simple sequence repeats. Genet. Resour. Crop Evol.

Lipka, A.E., Tian, F., Wang, Q., Peiffer, J., Li, M., Bradbury, P.J., et al. (2012) 
GAPIT: Genome association and prediction integrated tool. Bioinformatics.

McCormick, R.F., Truong, S.K., Sreedasyam, A., Jenkins, J., Shu, S., Sims, D., et al. (2018) The Sorghum bicolor reference genome: improved assembly, gene annotations, a transcriptome atlas, and signatures of genome organization. Plant J.

McKenna, A., Hanna, M., Banks, E., Sivachenko, A., Cibulskis, K., Kernytsky, A., et al. (2010) The genome analysis toolkit: A MapReduce framework for analyzing next-generation DNA sequencing data. Genome Res.

Montenegro, J.D., Golicz, A.A., Bayer, P.E., Hurgobin, B., Lee, H.T., Chan, C.K.K., et al. (2017) The pangenome of hexaploid bread wheat. Plant J., 90, 10071013.

Morris, G.P., Ramu, P., Deshpande, S.P., Hash, C.T., Shah, T., Upadhyaya, H.D., et al. (2013) Population genomic and genome-wide association studies of agroclimatic traits in sorghum. Proc. Natl. Acad. Sci. U. S. A.

Motlhaodi, T., Geleta, M., Bryngelsson, T., Fatih, M., Chite, S., and Ortiz, R. (2014) Genetic diversity in ex-situ conserved sorghum accessions of Botswana as estimated by: Microsatellite markers. Aust. J. Crop Sci.

Obilana, A.B., Rao, K.E.P., Mangombe, N., and House, L.R. (1996) Classification of sorghum races in the southern Africa sorghum germplasm.

Paradis, E., Claude, J., and Strimmer, K. (2004) APE: Analyses of phylogenetics and evolution in $R$ language. Bioinformatics.

Paterson, A.H., Bowers, J.E., Bruggmann, R., Dubchak, I., Grimwood, J., Gundlach, $\mathrm{H}$., et al. (2009) The Sorghum bicolor genome and the diversification of grasses. Nature.

Peng, Y., Leung, H.C.M., Yiu, S.M., and Chin, F.Y.L. (2012) IDBA-UD: A de novo assembler for single-cell and metagenomic sequencing data with highly uneven depth. Bioinformatics, 28, 1420-1428.

Price, H.J., Dillon, S.L., Hodnett, G., Rooney, W.L., Ross, L., and Johnston, J.S. (2005) Genome evolution in the genus Sorghum (Poaceae). In: Annals of Botany.

Ritter, K.B., McIntyre, C.L., Godwin, I.D., Jordan, D.R., and Chapman, S.C. (2007) An assessment of the genetic relationship between sweet and grain sorghums, within Sorghum bicolor ssp. bicolor (L.) Moench, using AFLP markers. Euphytica.

Sapkota, S., Boyles, R., Cooper, E., Brenton, Z., Myers, M., and Kresovich, S. (2020) Impact of sorghum racial structure and diversity on genomic prediction of grain yield components. Crop Sci.

Saxena, R.K., Edwards, D., and Varshney, R.K. (2014) Structural variations in plant genomes. Briefings Funct. Genomics Proteomics.

Schatz, M.C., Maron, L.G., Stein, J.C., Hernandez Wences, A., Gurtowski, J., Biggers, E., et al. (2014) Whole genome de novo assemblies of three divergent strains of rice, Oryza sativa, document novel gene space of aus and indica. 
Genome Biol.

Smit, AFA, Hubley, R \& Green, P. RepeatMasker.

The Races of Sorghum in Africa (2012) In: Origins of African Plant Domestication.

Tian, T., You, Q., Zhang, L., Yi, X., Yan, H., Xu, W., and Su, Z. (2016) SorghumFDB: sorghum functional genomics database with multidimensional network analysis. Database (Oxford).

Trapnell, C., Williams, B.A., Pertea, G., Mortazavi, A., Kwan, G., Van Baren, M.J., et al. (2010) Transcript assembly and quantification by RNA-Seq reveals unannotated transcripts and isoform switching during cell differentiation. Nat. Biotechnol.

Upadhyaya, H.D., Reddy, K.N., Vetriventhan, M., Gumma, M.K., Irshad Ahmed, M., Manyasa, E., et al. (2017) Geographical distribution, diversity and gap analysis of East African sorghum collection conserved at the ICRISAT genebank. Aust. J. Crop Sci.

Usha Kiranmayee, K.N.S., Hash, C.T., Sivasubramani, S., Ramu, P., Amindala, B.P., Rathore, A., et al. (2020) Fine-mapping of sorghum stay-green qtl on chromosome10 revealed genes associated with delayed senescence. Genes (Basel).

Valluru, R., Gazave, E.E., Fernandes, S.B., Ferguson, J.N., Lozano, R., Hirannaiah, P., et al. (2019) Deleterious mutation burden and its association with complex traits in sorghum (Sorghum bicolor). Genetics.

Varoquaux, N., Cole, B., Gao, C., Pierroz, G., Baker, C.R., Patel, D., et al. (2019) Transcriptomic analysis of field-droughted sorghum from seedling to maturity reveals biotic and metabolic responses. Proc. Natl. Acad. Sci. U. S. A.

Wang, W., Mauleon, R., Hu, Z., Chebotarov, D., Tai, S., Wu, Z., et al. (2018) Genomic variation in 3,010 diverse accessions of Asian cultivated rice. Nature.

Woldesemayat, A.A. and Ntwasa, M. (2018) Pathways and Network Based Analysis of Candidate Genes to Reveal Cross-Talk and Specificity in the Sorghum (Sorghum bicolor (L.) Moench) Responses to Drought and It's Co-occurring Stresses. Front. Genet.

Yan, S., Wang, L., Zhao, L., Wang, H., and Wang, D. (2018) Evaluation of Genetic Variation among Sorghum Varieties from Southwest China via Genome Resequencing. Plant Genome.

Yano, K., Yamamoto, E., Aya, K., Takeuchi, H., Lo, P., Hu, L., et al. (2016) Genomewide association study using whole-genome sequencing rapidly identifies new genes influencing agronomic traits in rice. Nat. Genet., 48, 927-934.

Yao, W., Li, G., Zhao, H., Wang, G., Lian, X., and Xie, W. (2015) Exploring the rice dispensable genome using a metagenome-like assembly strategy. Genome Biol., 16, 187.

Zhao, J., Bayer, P.E., Ruperao, P., Saxena, R.K., Khan, A.W., Golicz, A.A., et al. (2020) Trait associations in the pangenome of pigeon pea (Cajanus cajan). Plant Biotechnol. J. 
Zheng, L.-Y., Guo, X.-S., He, B., Sun, L.-J., Peng, Y., Dong, S.-S., et al. (2011) Genome-wide patterns of genetic variation in sweet and grain sorghum (Sorghum bicolor). Genome Biol., 12, R114.

Zhou, P., Silverstein, K.A.T., Ramaraj, T., Guhlin, J., Denny, R., Liu, J., et al. (2017) Exploring structural variation and gene family architecture with De Novo assemblies of 15 Medicago genomes. BMC Genomics, 18, 261. 


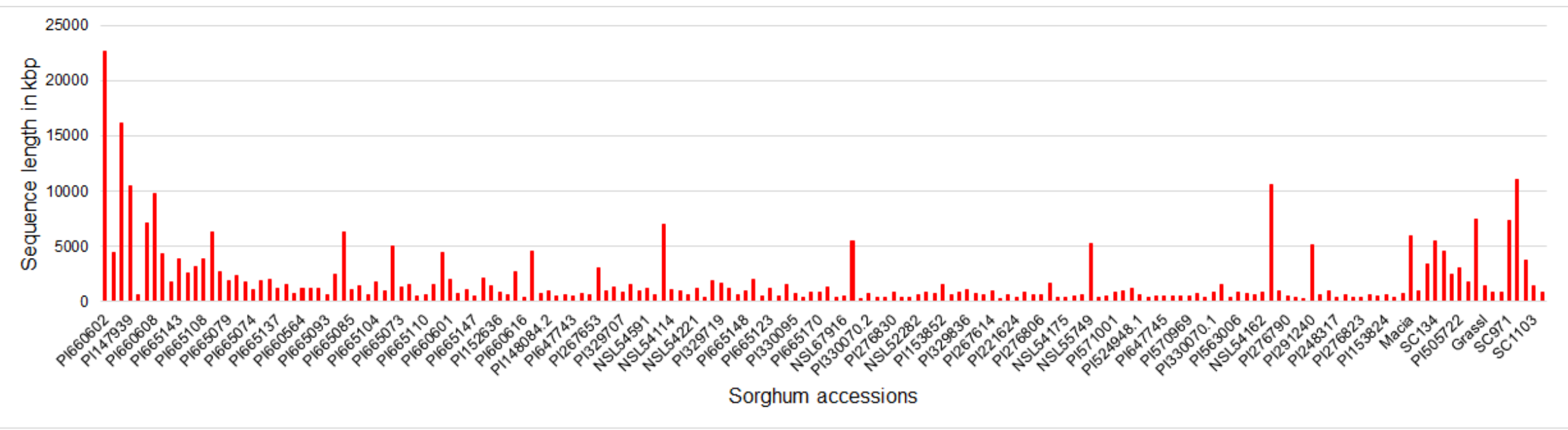

Supplementary Figure 1: Whole genome sequence of 176 sorghum accessions mapped iteratively to the updated reference sequence assembly and the unmapped sequence reads were assembly iteratively. The plot represents the size of the sequence assembly gained from respective accessions. 


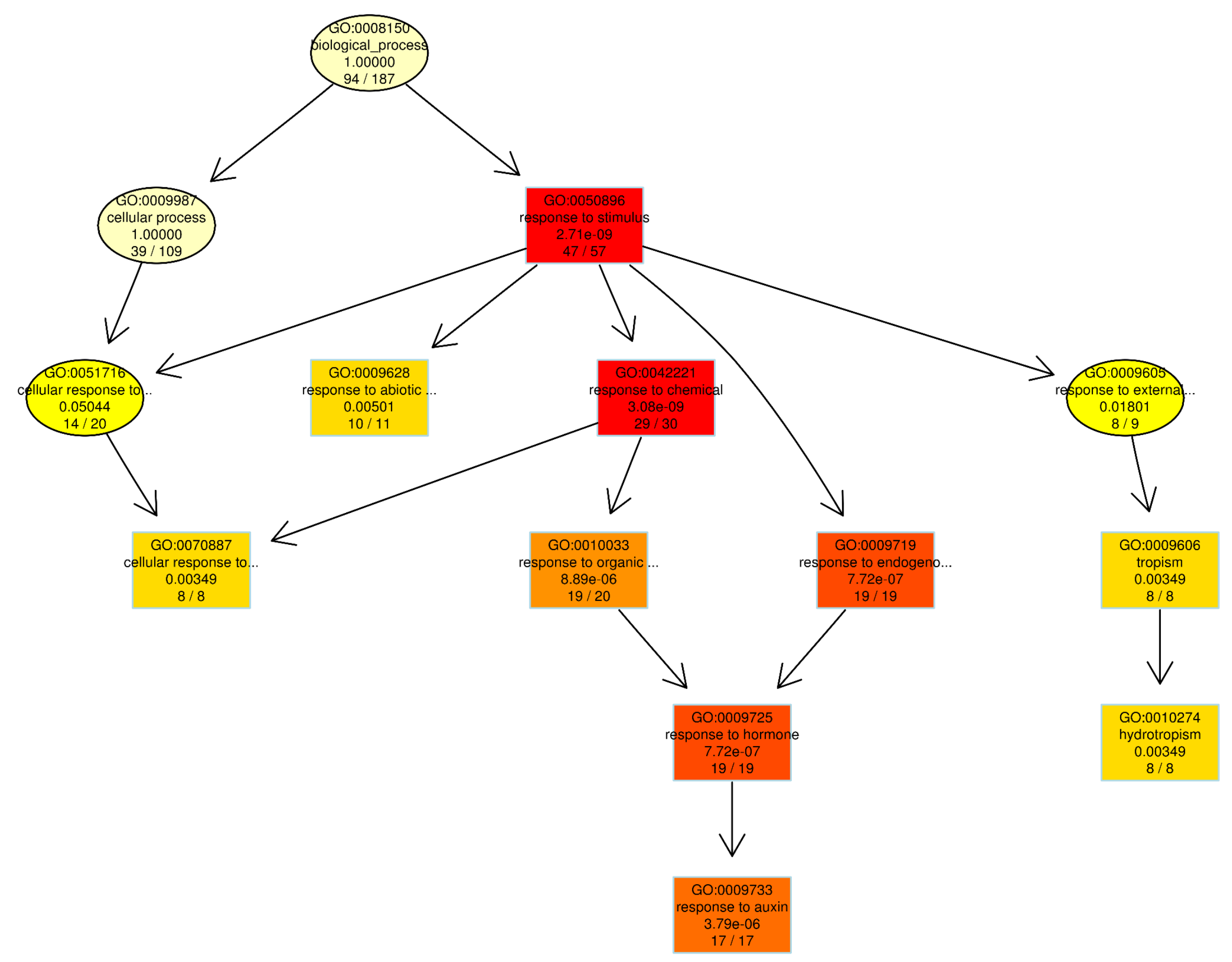




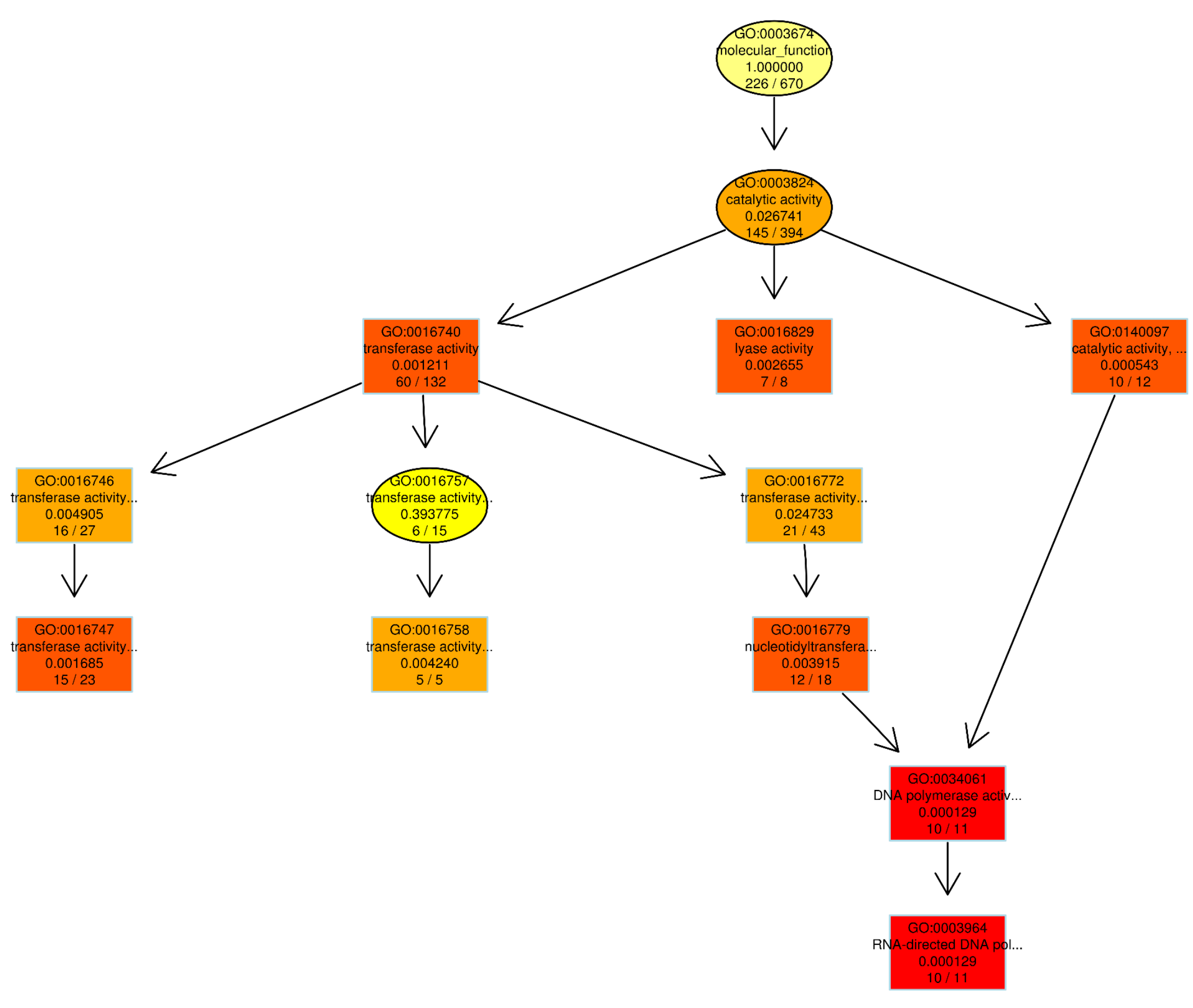




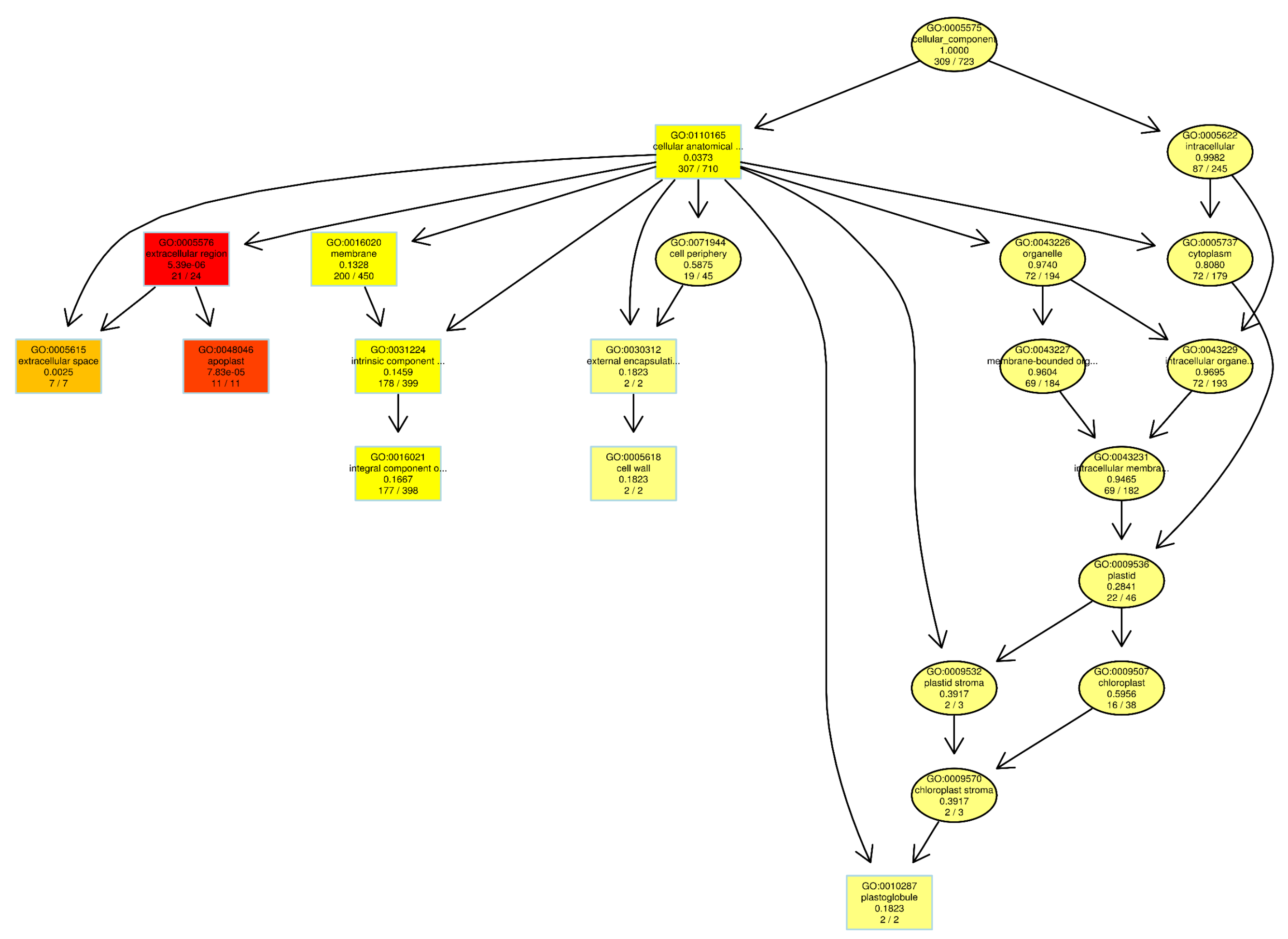

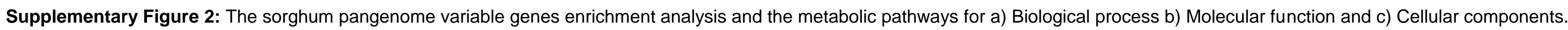
Top $10 \mathrm{GO}$ terms identified for scoring GO terms for enrichment. Rectangles indicate the 10 most significant terms and the colour represents the relative significance ranging from red (most significant) to yellow (least significant). Each node has GO identifier and name with raw p-values and number of significant genes out of total genes annotated. 


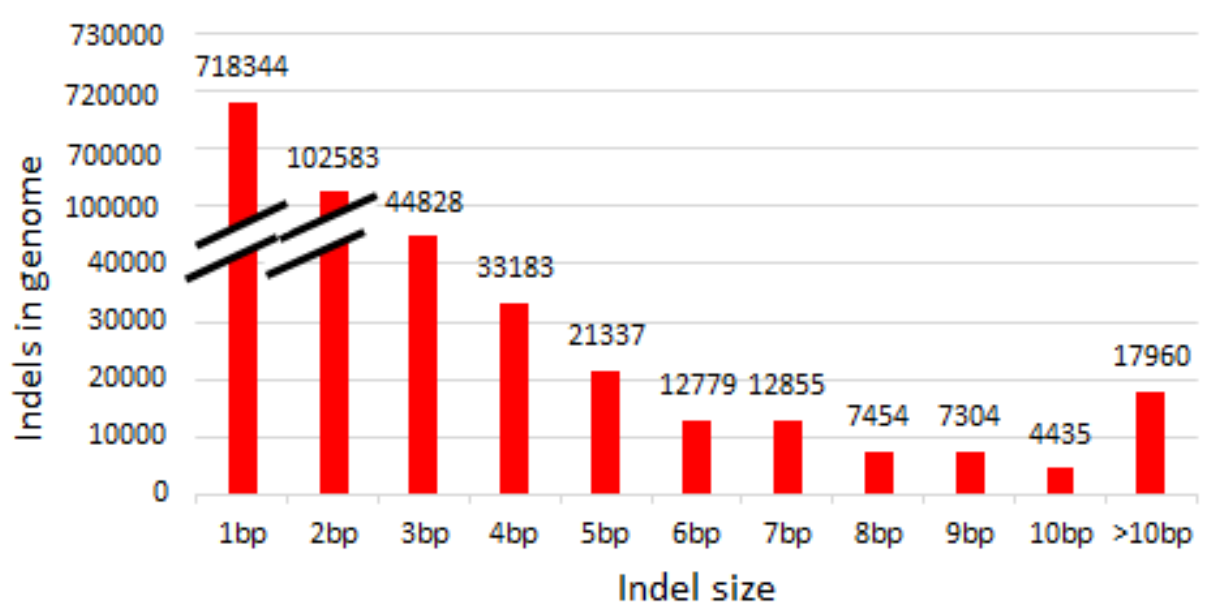

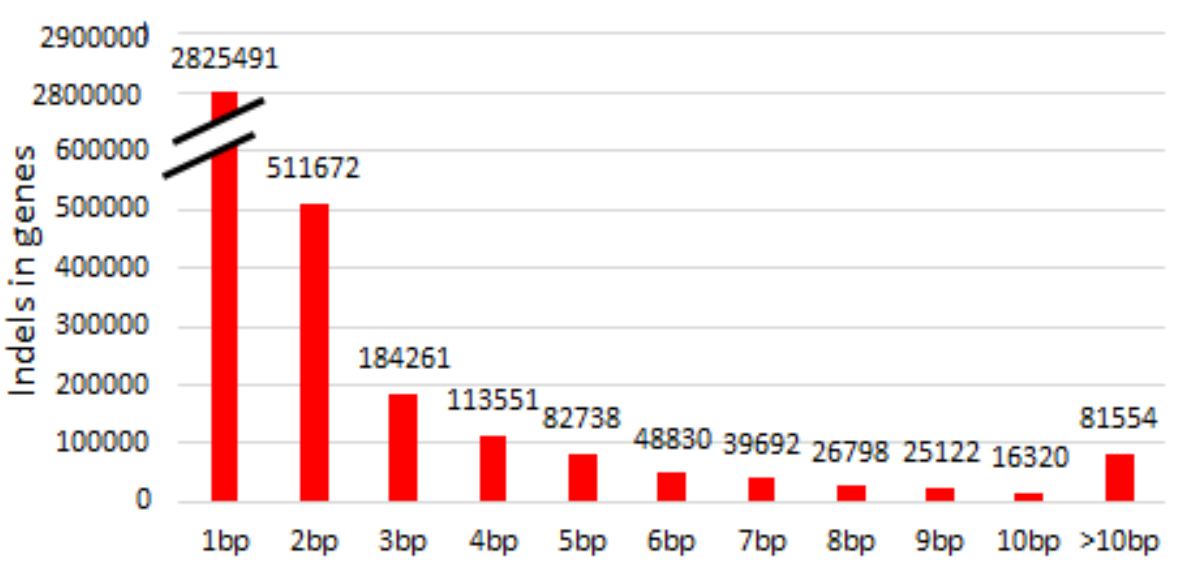

Indel size

Supplementary Figure 3: Insertions and deletions of various size distribution at sorghum pangenome a) genome level and b) gene level 


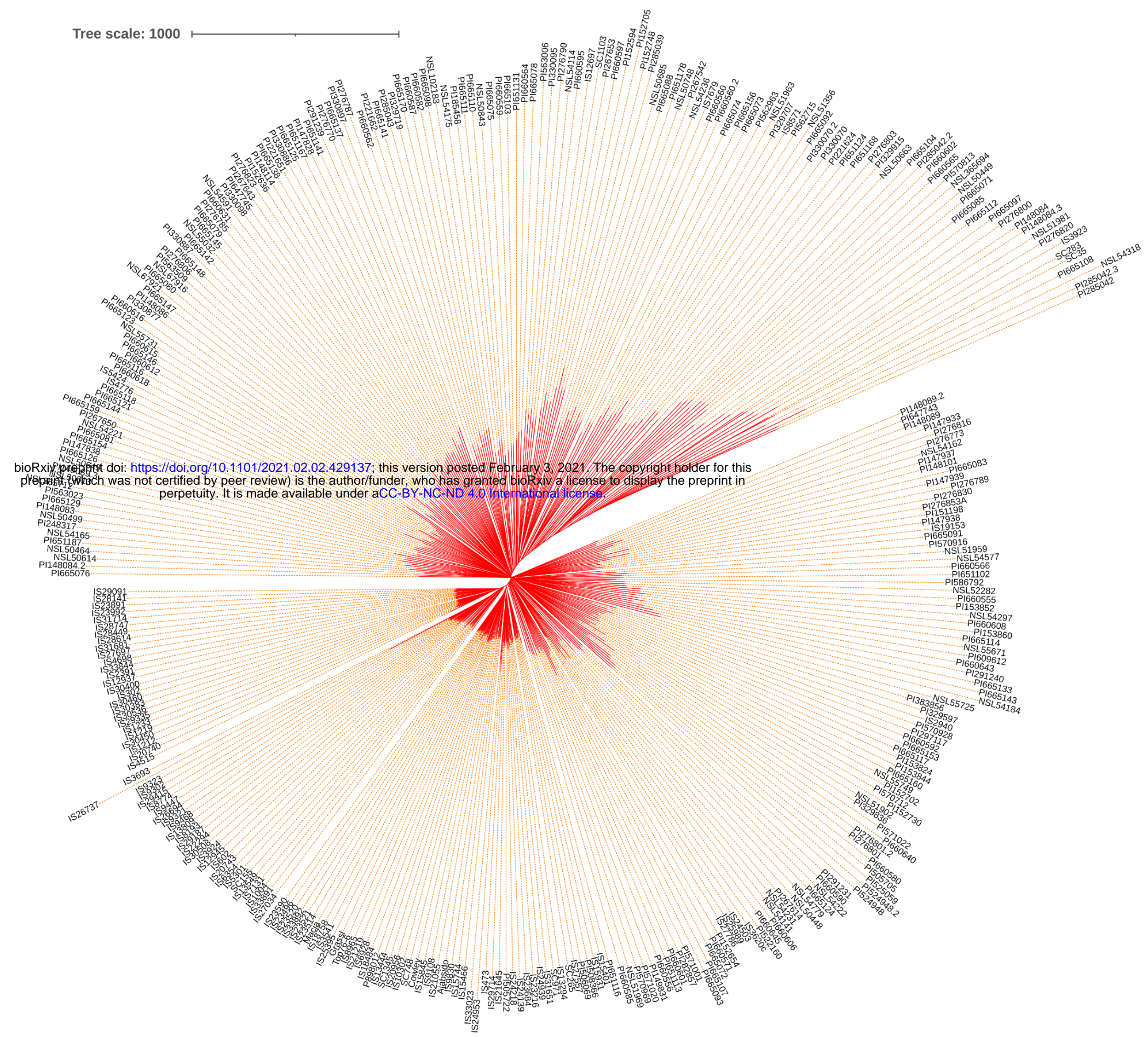

Supplementary Figure 4: The genetic relationship of 354 sorghum accession neighbour joining (with 1000 bootstrap) analysis (the unrooted tree with branch length in red colour). 


\section{Chromosome1}

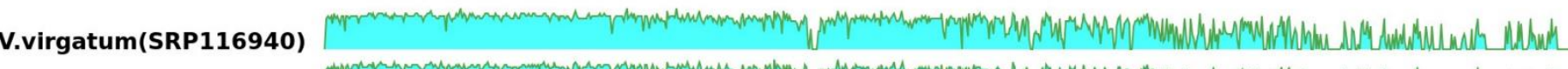
Tiandougou(SRP116938)

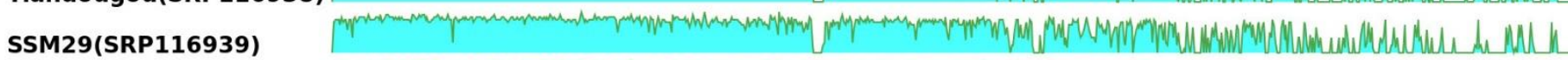

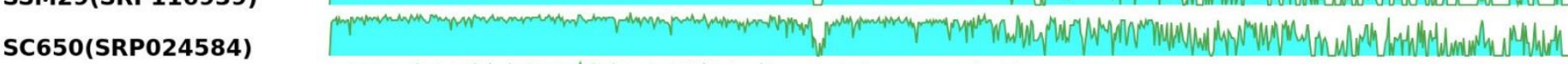

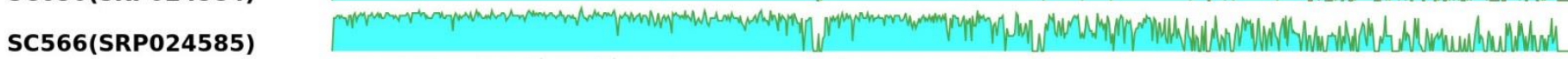

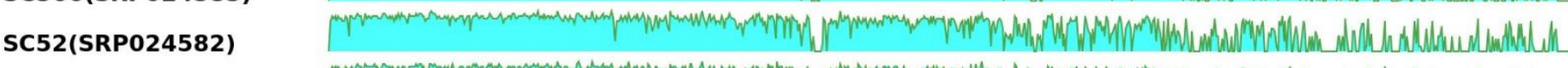

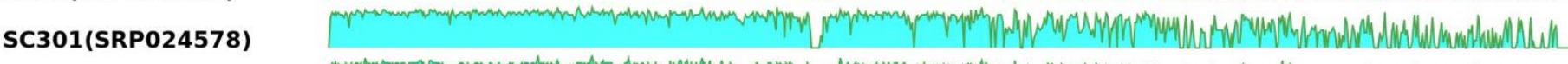

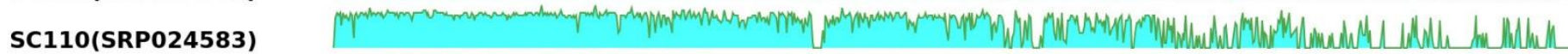

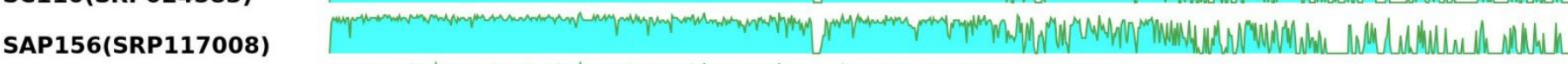

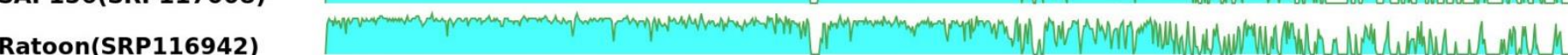

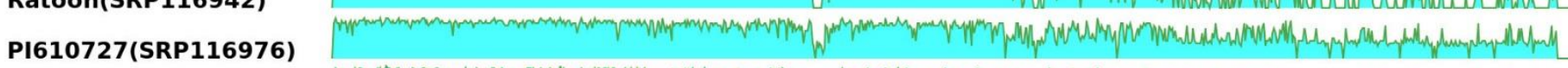
LiangTangAi(SRP015362)

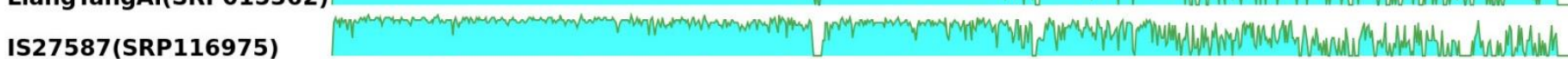
IS25702(SRP024581) Cr

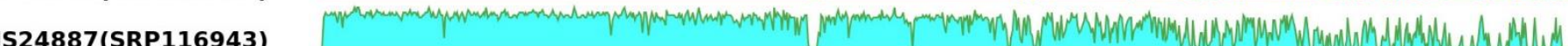

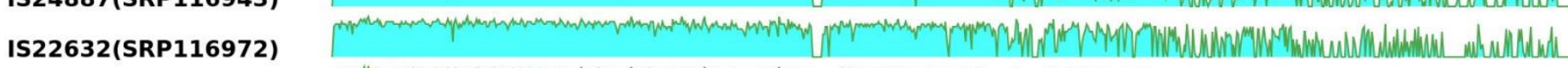

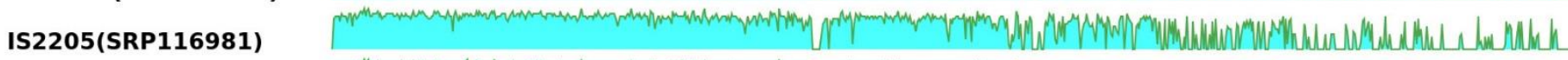

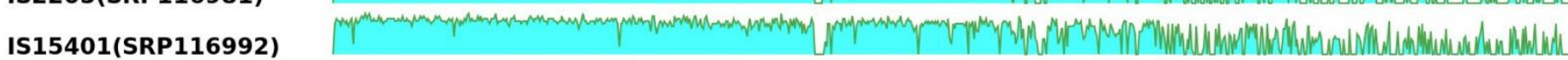

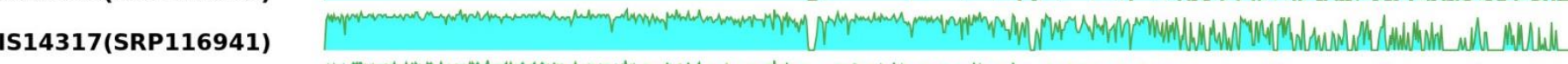
IS1054(M35-1)(SRP116995)

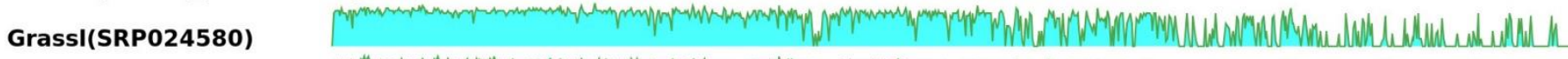

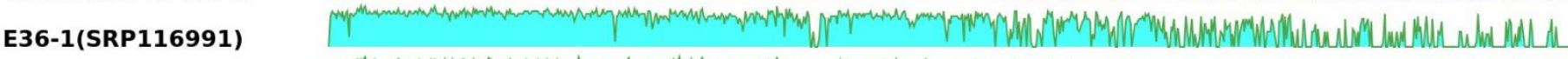

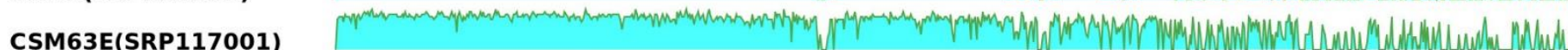

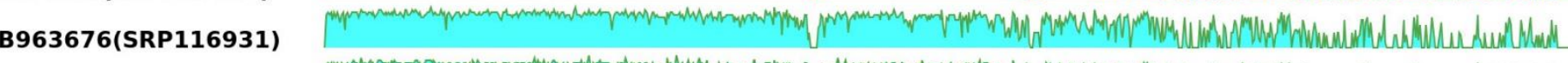

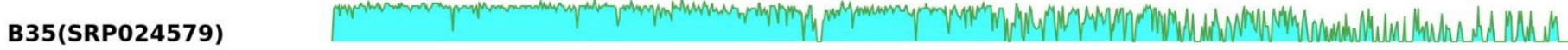

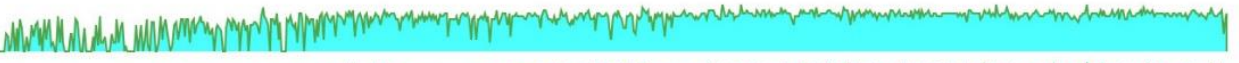

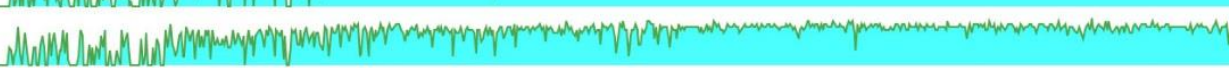

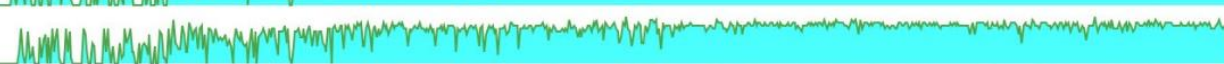

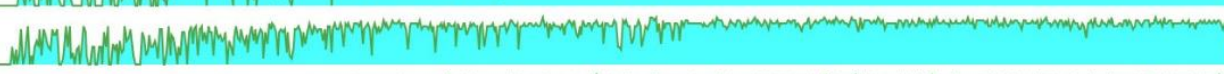

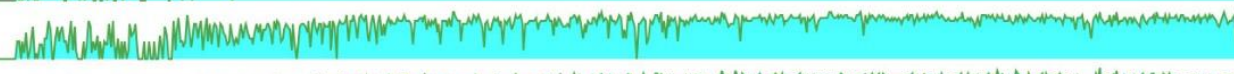

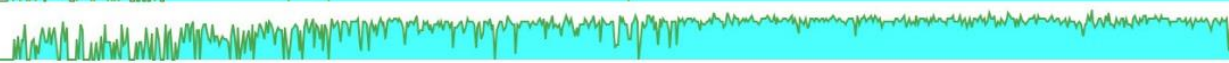

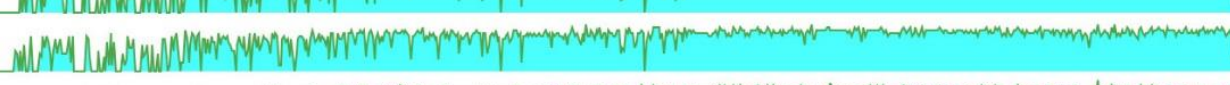

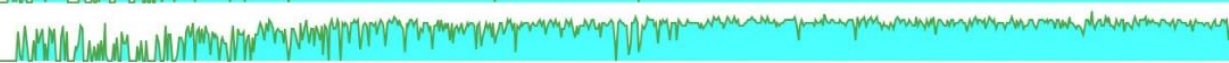

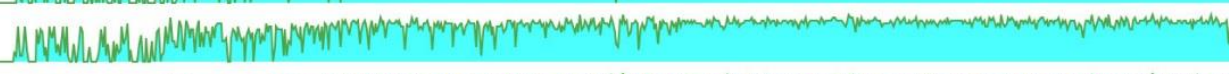

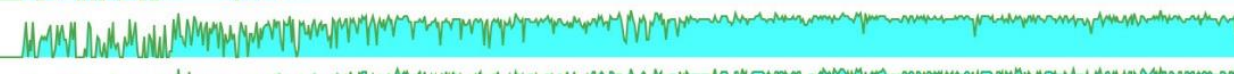
How

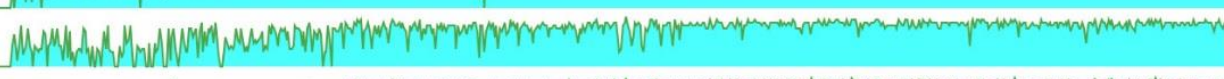

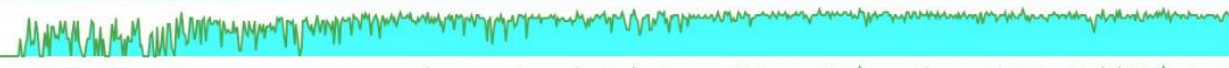

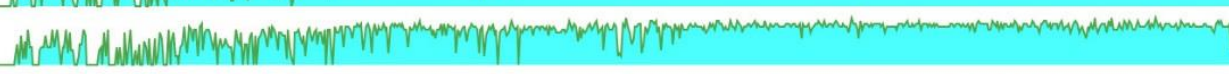

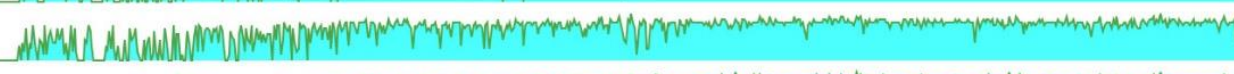

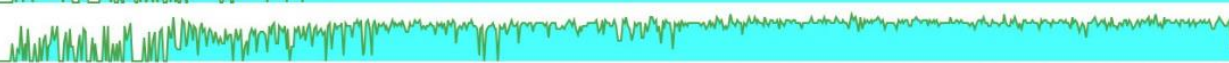

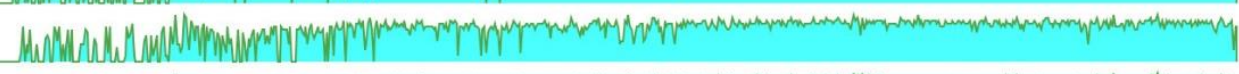

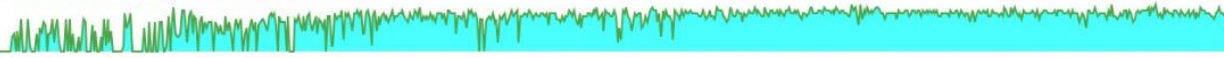

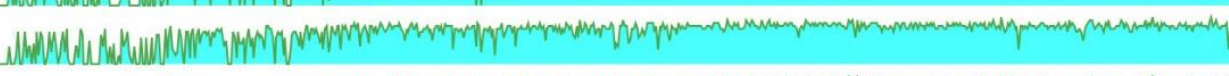

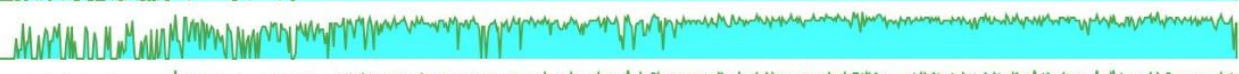

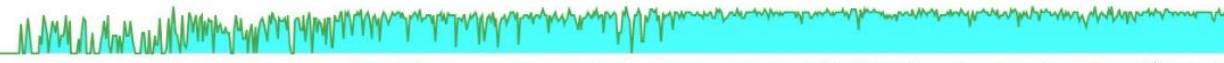

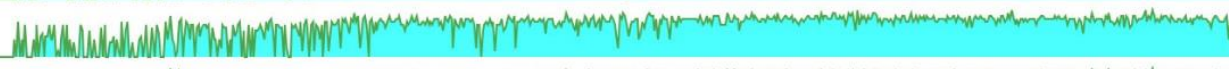

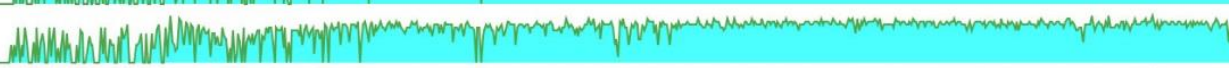

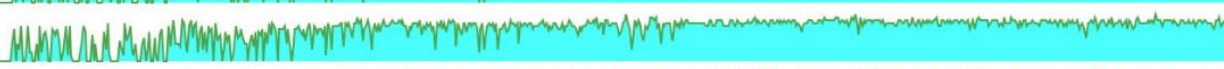

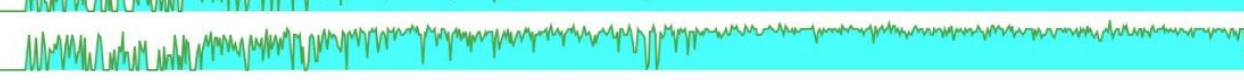

Chro1-genes

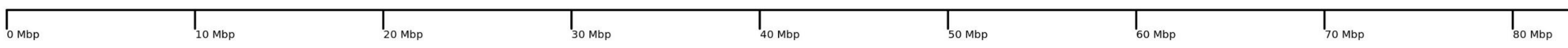




\title{
Chromosome2
}

V.virgatum(SRP116940)

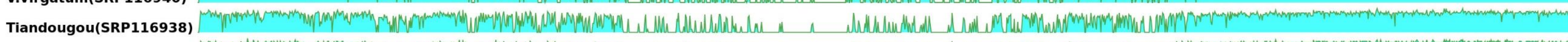

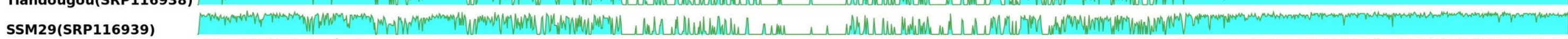
SC650(SRP024584) SC566(SRP024585) SC52(SRP024582) SC301(SRP024578) SC110(SRP024583) SAP156(SRP117008) Ratoon(SRP116942) PI610727(SRP116976) LiangTangAi(SRP015362) IS27587(SRP116975) (S25702(SRP024581) IS24887(SRP116943) IS22632(SRP116972) IS2205(SRP116981) IS15401(SRP116992) S14317(SRP116941) 1695)

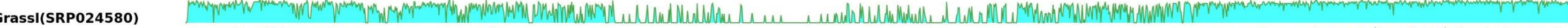

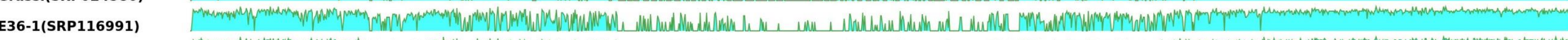

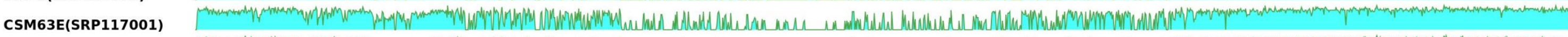

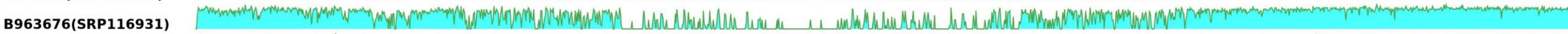
B35(SRP024579)

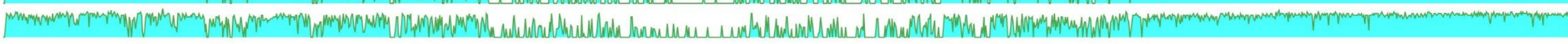

Chro2-genes

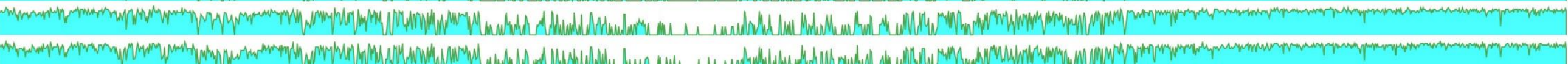

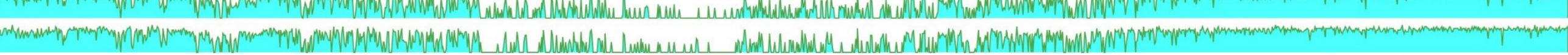

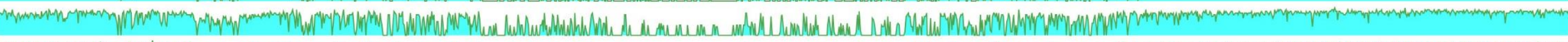

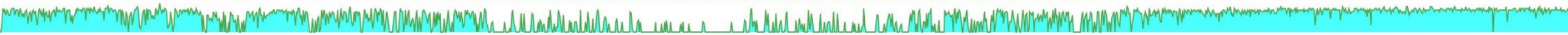

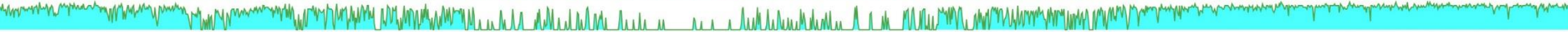

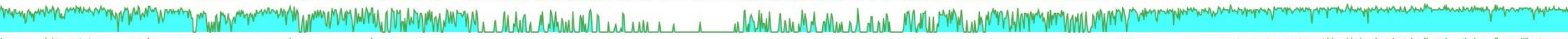

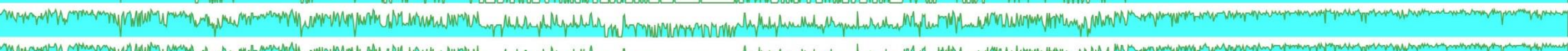

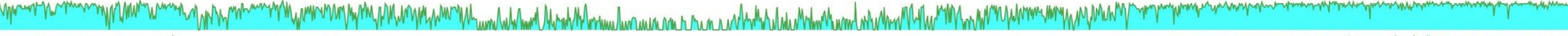

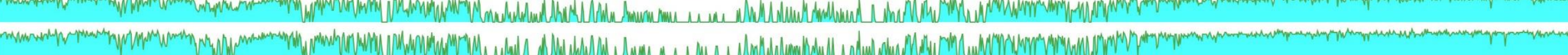

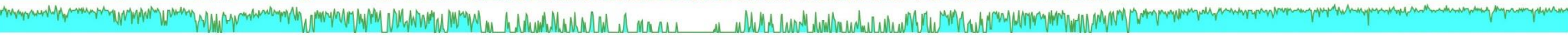

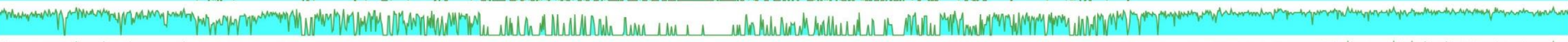

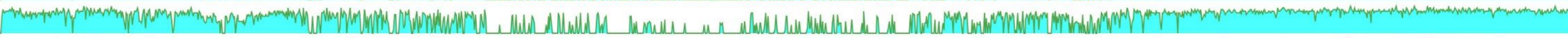

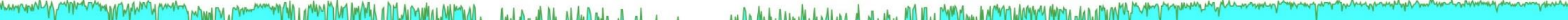

(risp

\begin{abstract}
(2)
\end{abstract}

\begin{abstract}
(2)
\end{abstract}

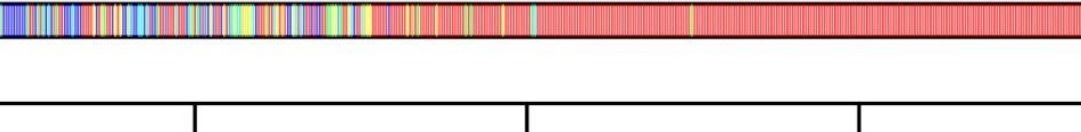

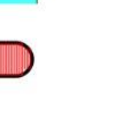


c.

\section{Chromosome3}

V.virgatum(SRP116940) Tiandougou(SRP116938)

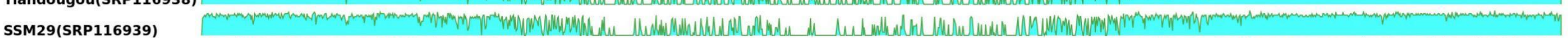

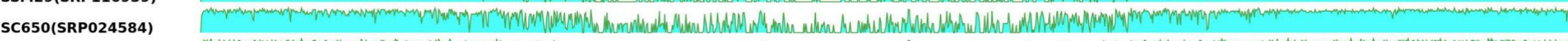

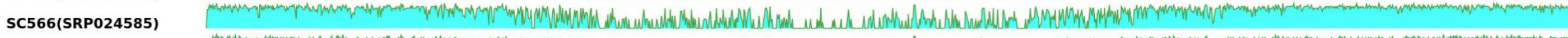

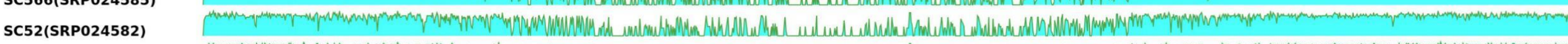

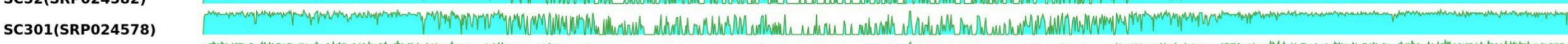

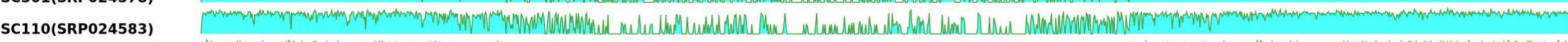

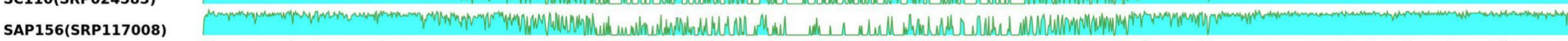

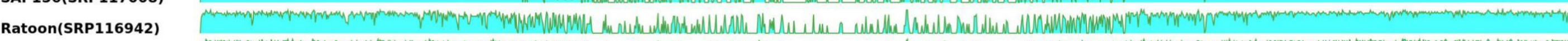
P1610727(SRP116976) LiangTangAi(SRP015362)

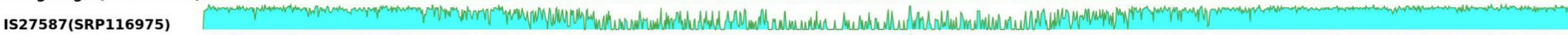

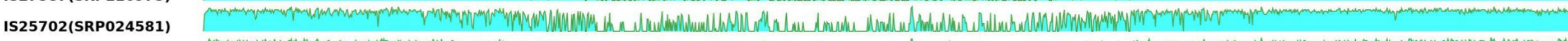

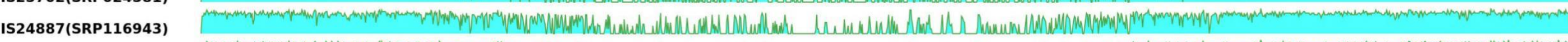

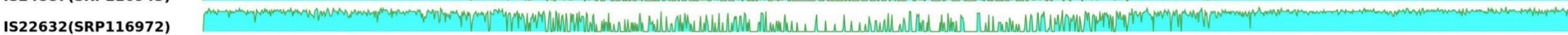

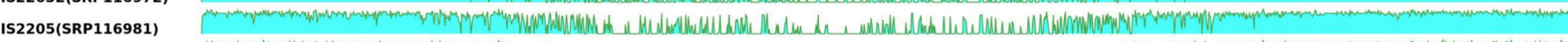

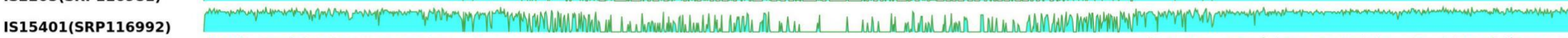

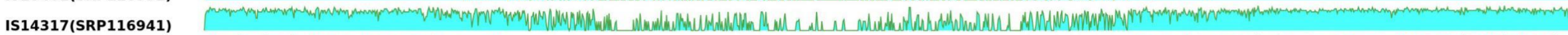
IS1054(M35-1)(SRP116995)

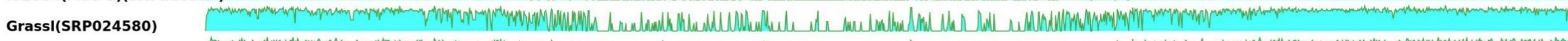

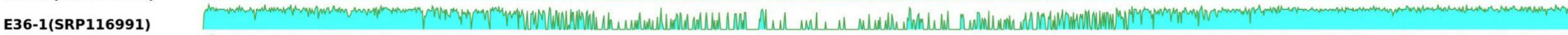

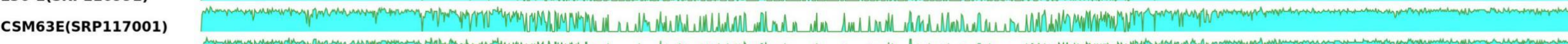
B963676(SRP116931) D C $335(58 P 024579)$

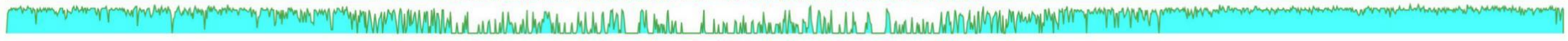

Chro3-genes

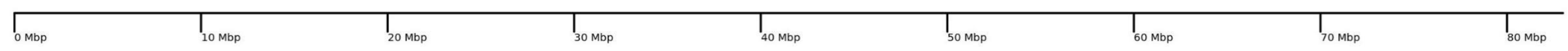




\section{Chromosome4}

V.virgatum(SRP116940) Tiandougou(SRP116938)

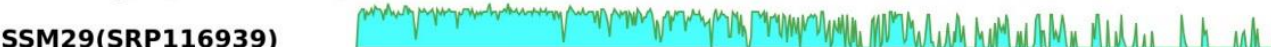

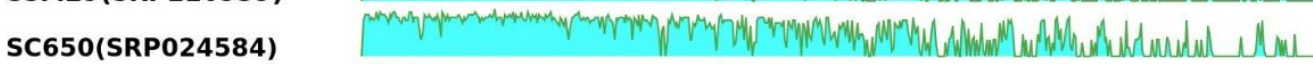

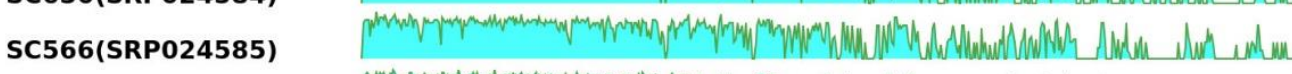

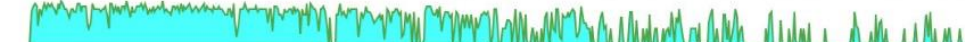

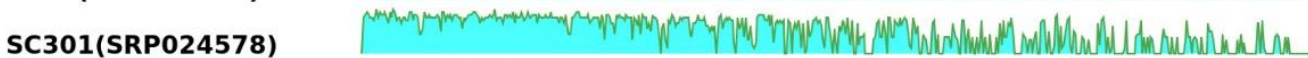

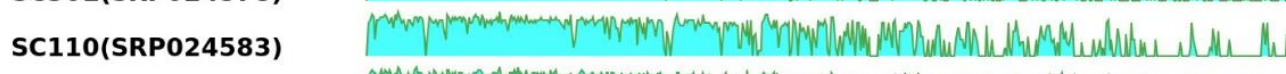

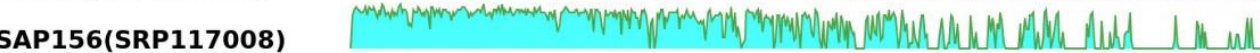
Satoonisposin P1610727(SRP116976) LiangTangAi(SRP015362) IS27587(SRP116975) IS25702(SRP024581) IS24887(SRP116943) IS22632(SRP116972) IS2205(SRP116981) IS15401(SRP116992) IS14317(SRP116941) IS1054(M35-1)(SRP116 GrassI(SRP024580) E36-1(SRP116991) CSM63E(SRP117001) B963676(SRP116931) B35(SRP024579)

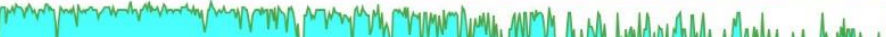

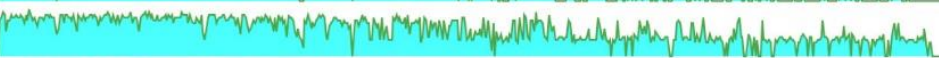

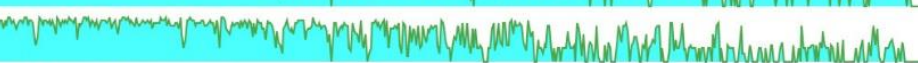

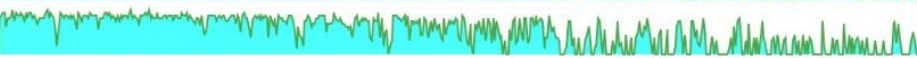

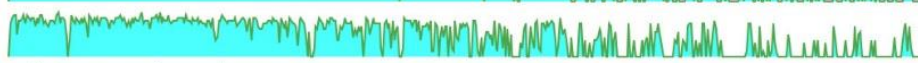

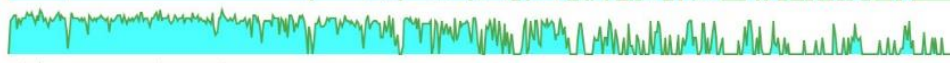

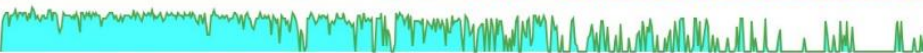

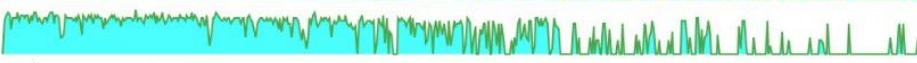

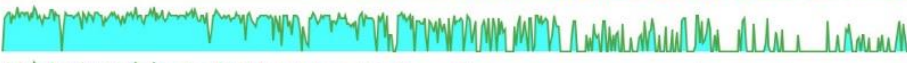

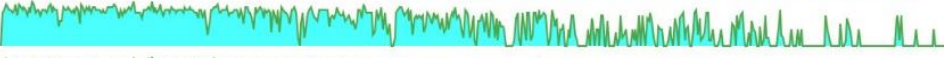
5)

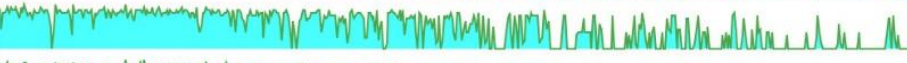
.

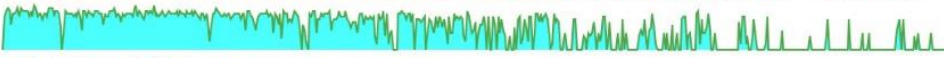

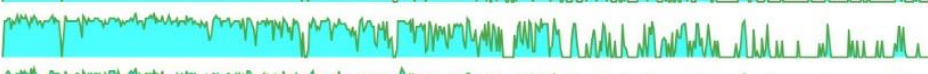

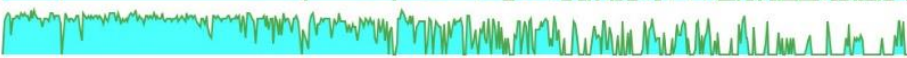

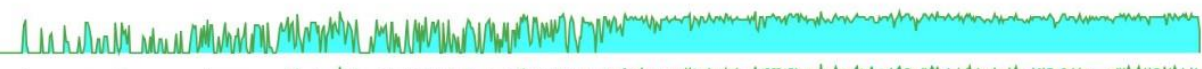

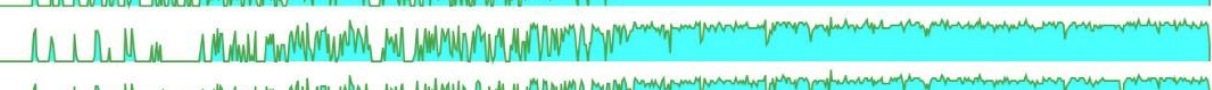

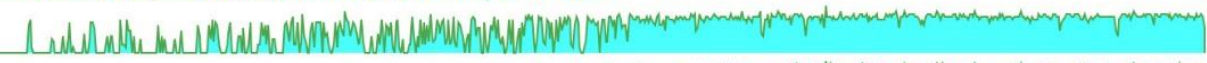

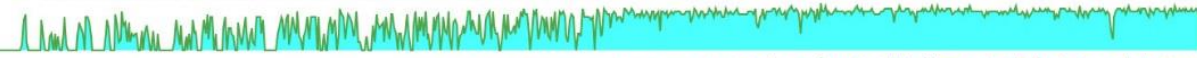

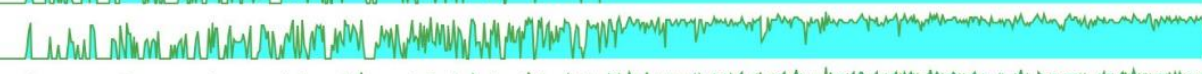

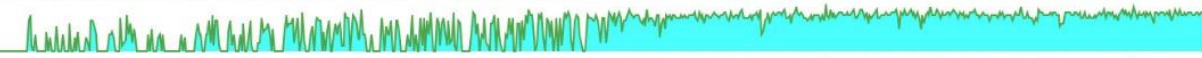

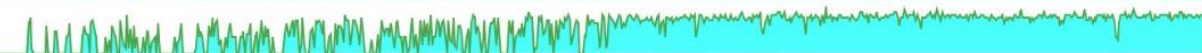

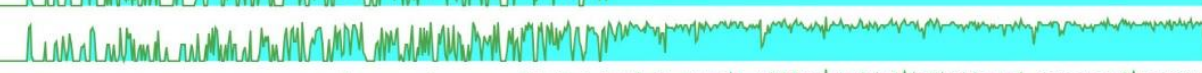

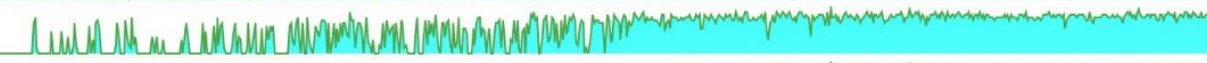

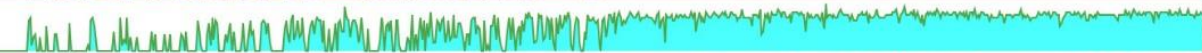

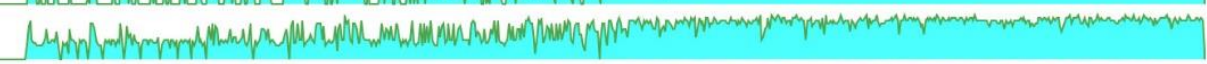

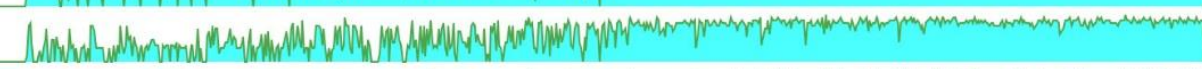

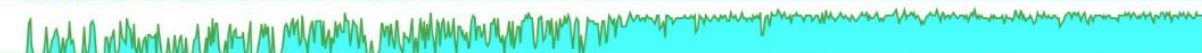

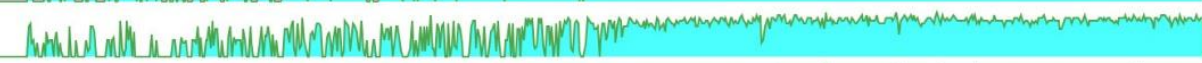

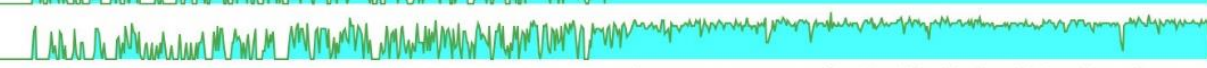

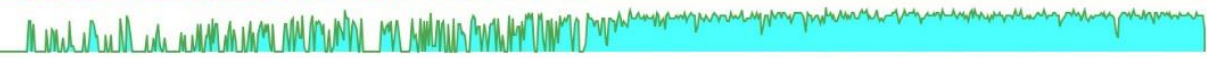

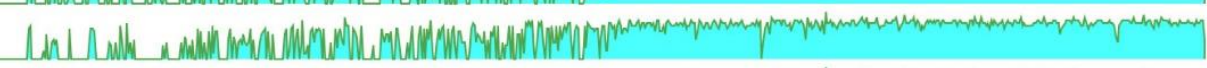

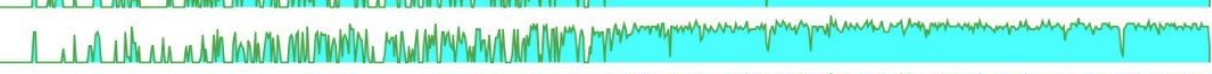

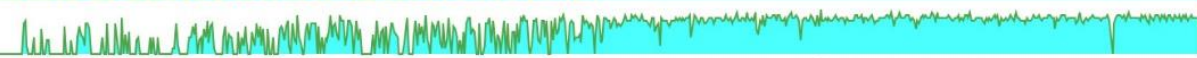

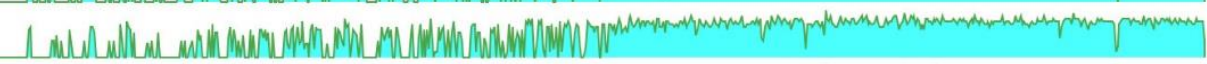

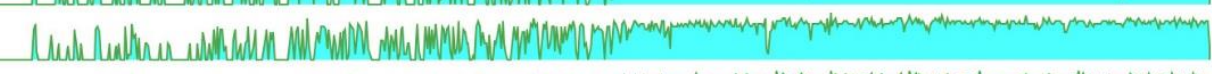

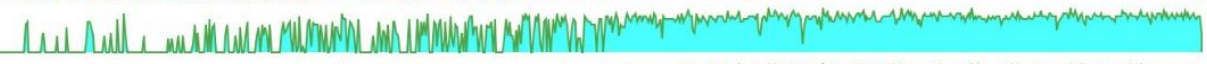

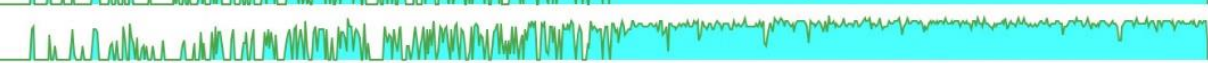

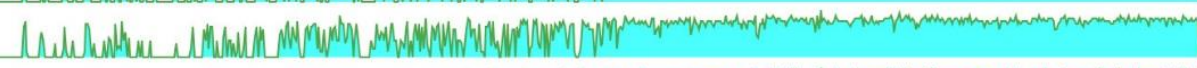

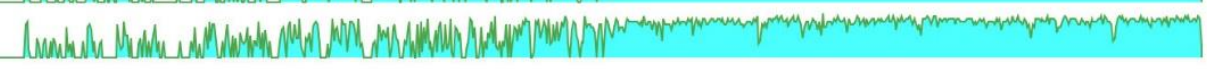

Chro4-genes

$\Gamma_{\text {Mbp }}$ $T_{10 \text { Mbp }}$

\begin{abstract}
$T_{20 \text { Mbp }}$
\end{abstract}
$T_{30 \mathrm{Mbp}}$

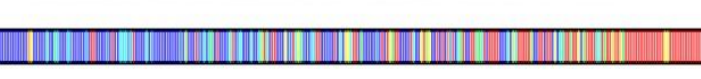

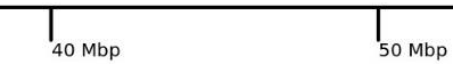

50 Mbp
$T_{60 \mathrm{Mbp}}$
$T_{70 \mathrm{Mbp}}$ 


\section{Chromosome5}

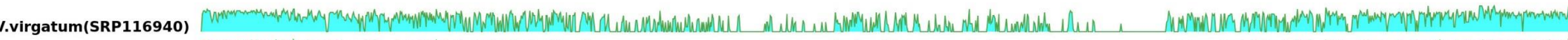

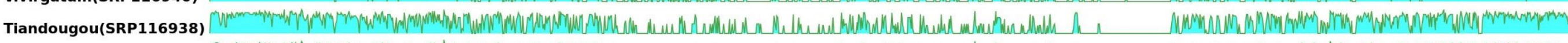

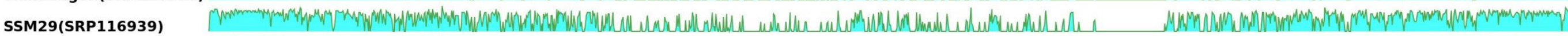

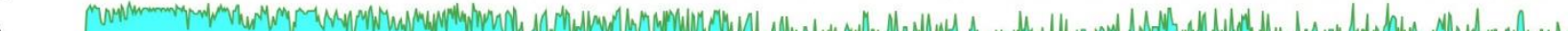

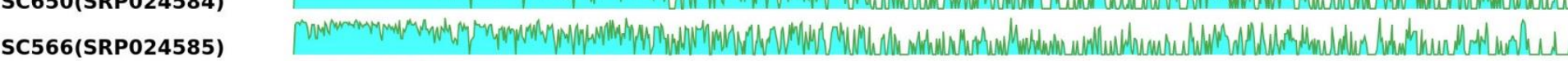

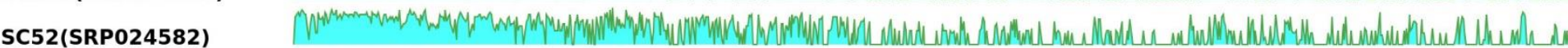
SC301(SRP024578) SC110(SRP024583)

SAP156(SRP117008 Ratoon(SRP116942)

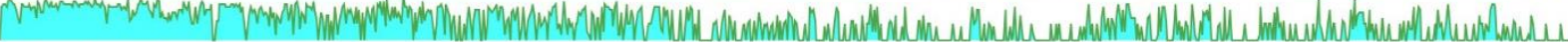

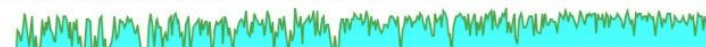

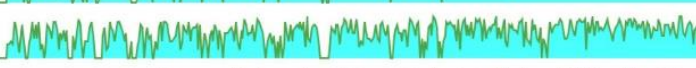
W

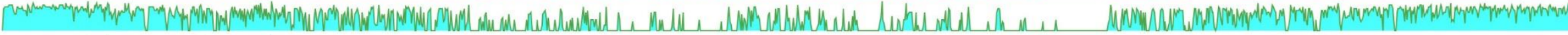

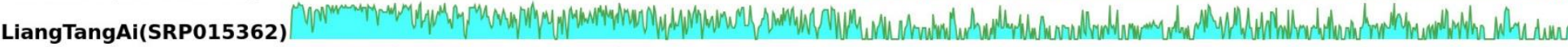

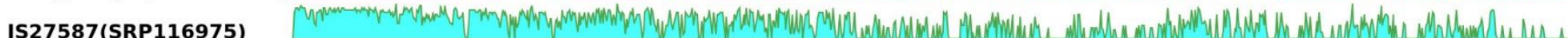

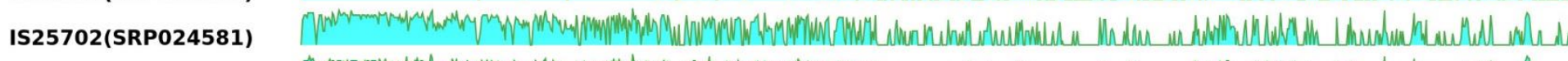

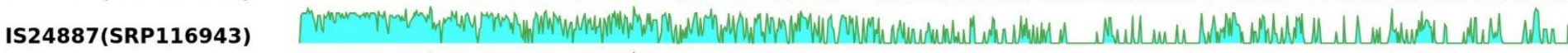

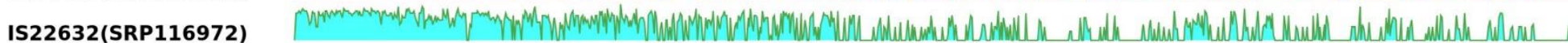

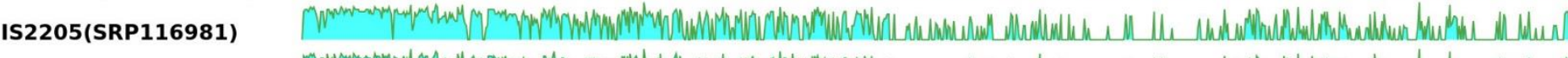

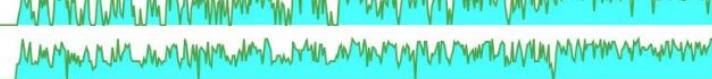

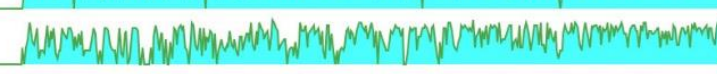

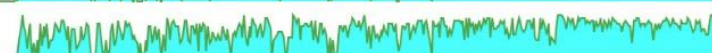

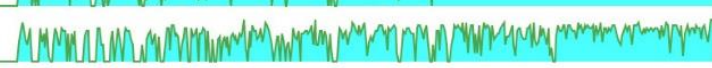

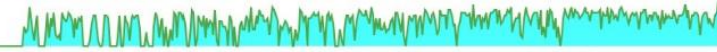

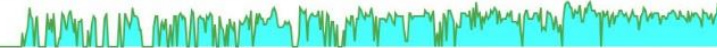

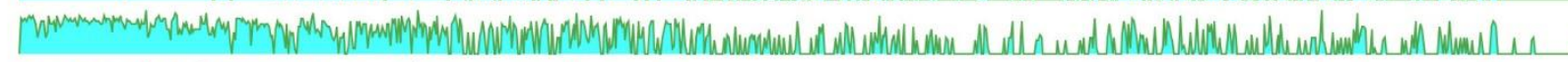

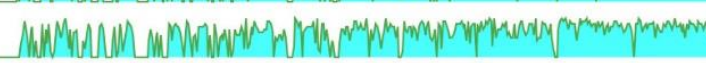

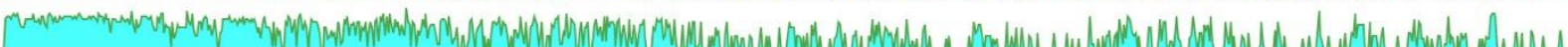

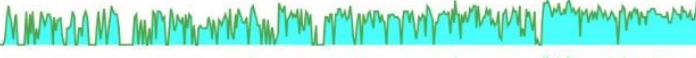
IS14317(SRP116941) IS1054(M35-1)(SRP11699 政

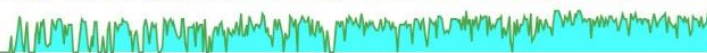

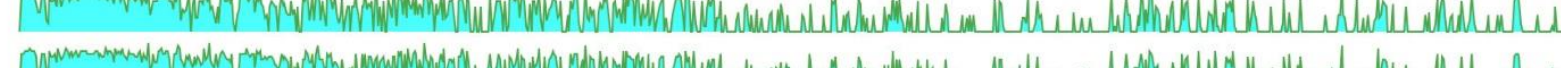

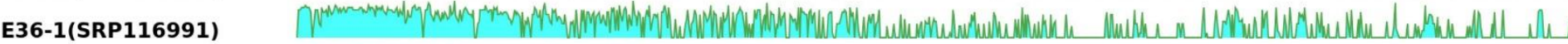

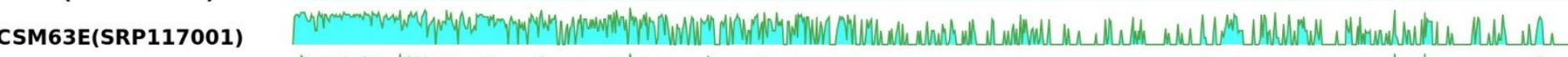

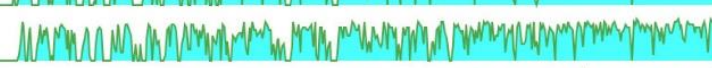
B963676(SRP116931) B35(SRP024579)

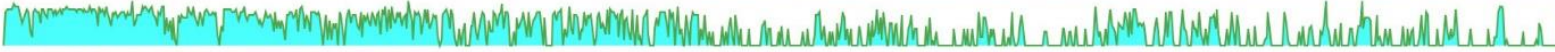

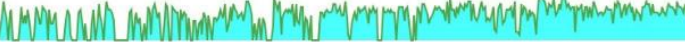

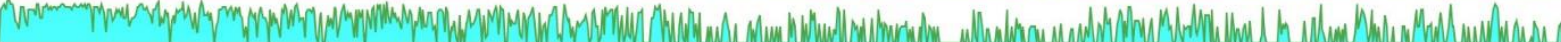

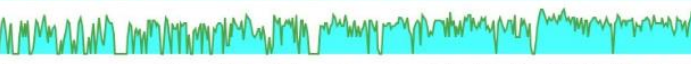

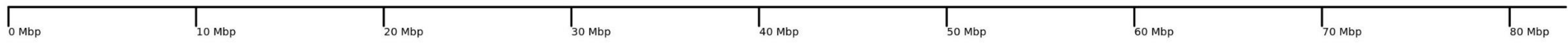




\section{Chromosome6}

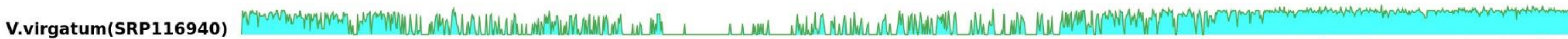

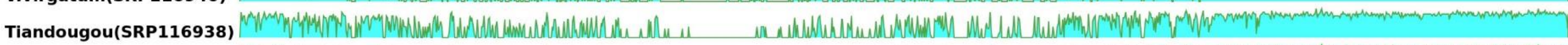

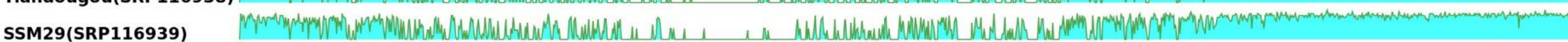

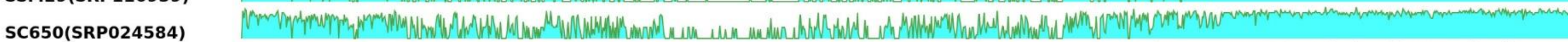
SC566(SRP024585) SC52(SRP024582) SC301(SRP024578) SC110(SRP024583) SAP156(SRP117008) Ratoon(SRP116942)

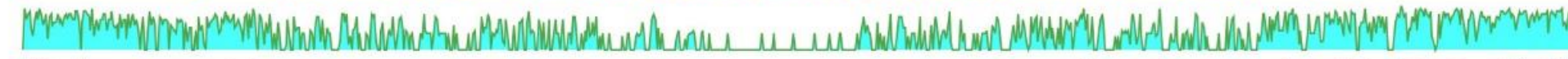

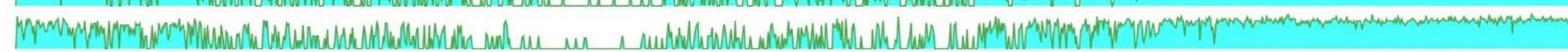

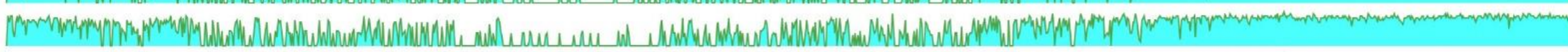

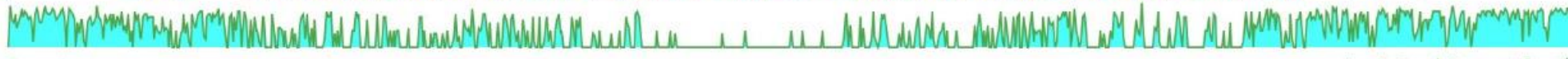

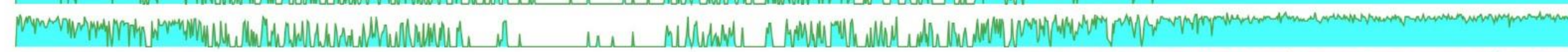

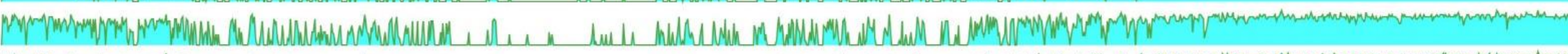
P1610727(SRP116976)

LiangTangAi(SRP015362 IS27587(SRP116975) IS25702(SRP024581) IS24887(SRP116943) IS22632(SRP116972) IS2205(SRP116981) IS15401(SRP116992) IS14317(SRP116941) IS1054(M35-1)(SRP1169 GrassI(SRP024580) E36-1(SRP116991)

CSM63E(SRP117001) B963676(SRP116931) B35(SRP024579)

Chro6-genes

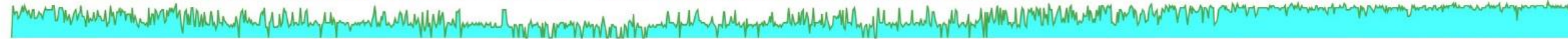

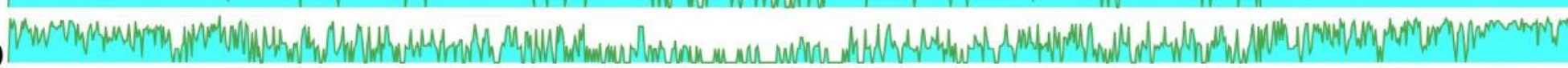

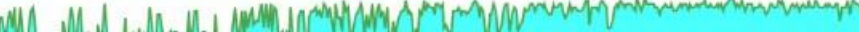
2

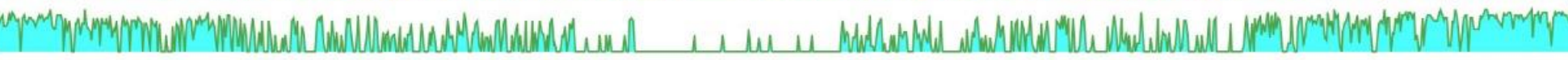

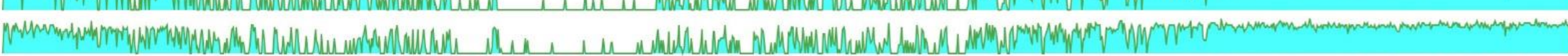

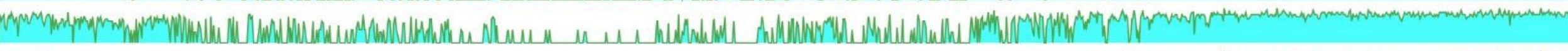

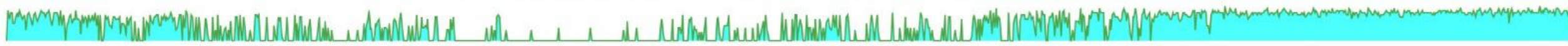

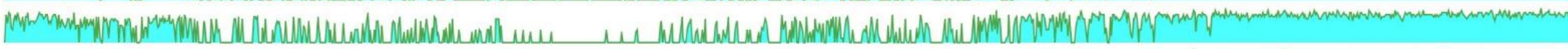

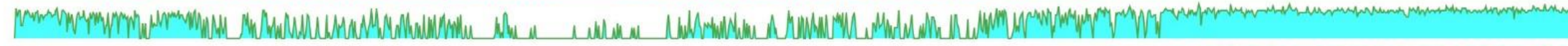

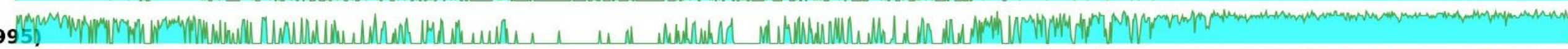

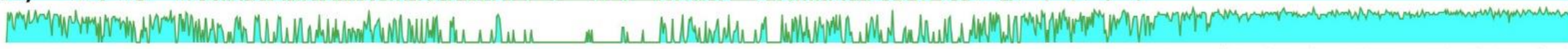

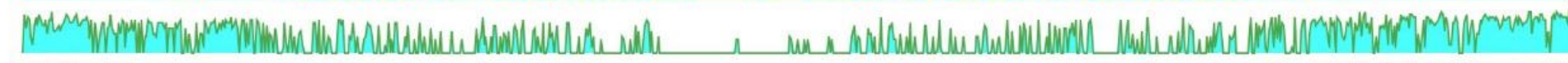

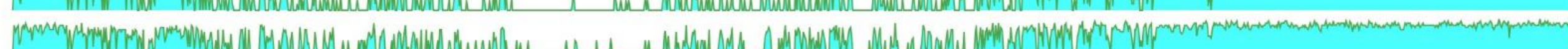

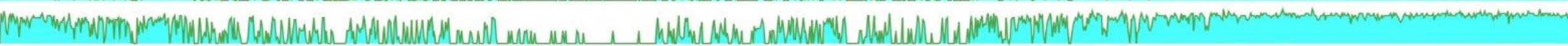

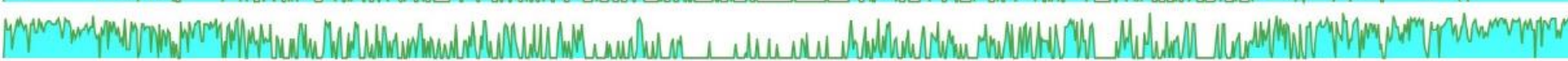

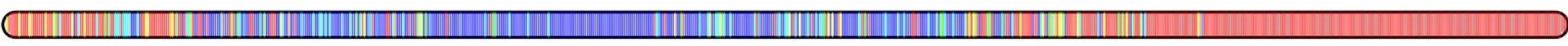

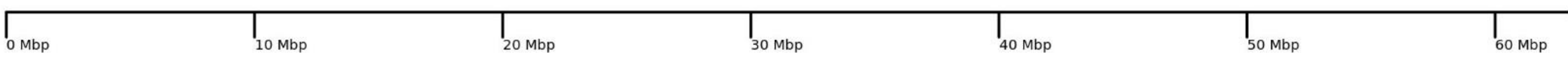




\section{Chromosome7}

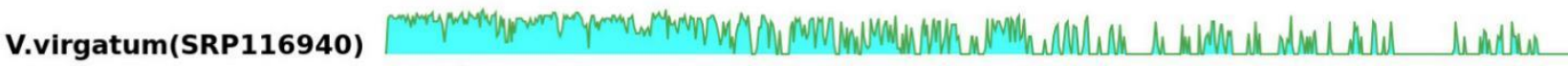

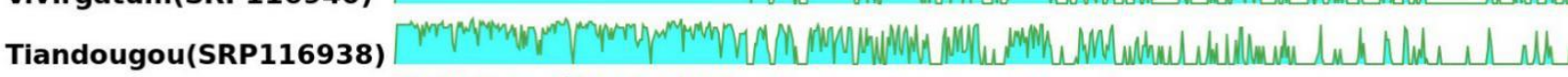

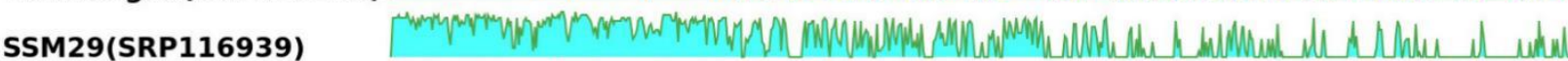

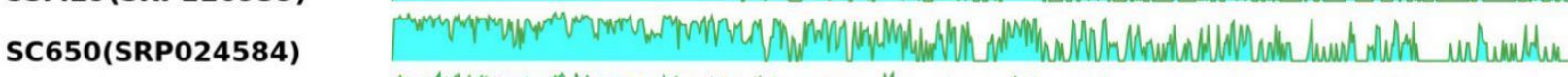

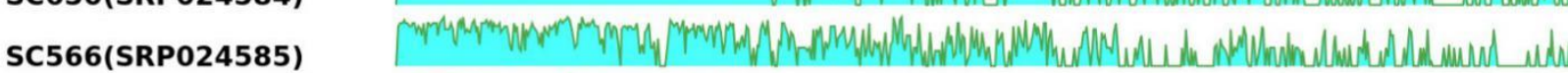

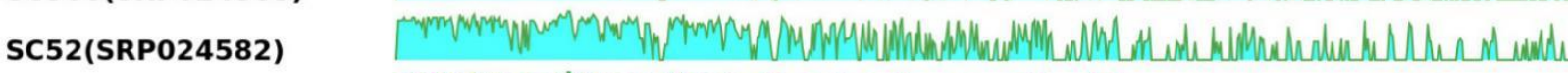

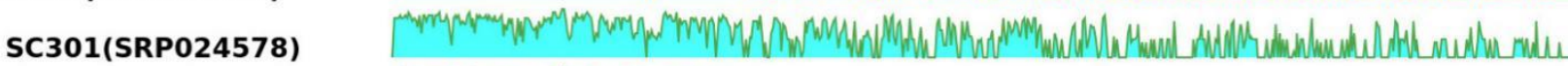

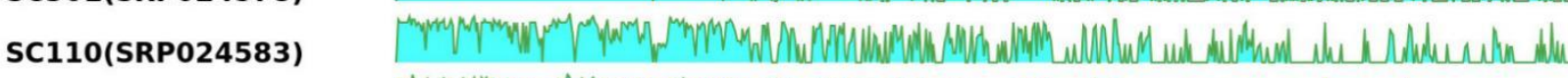

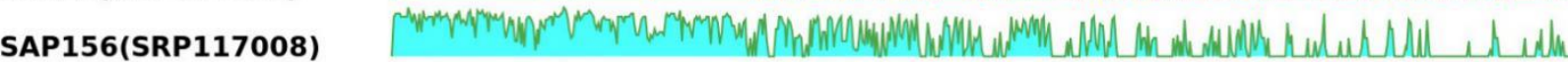

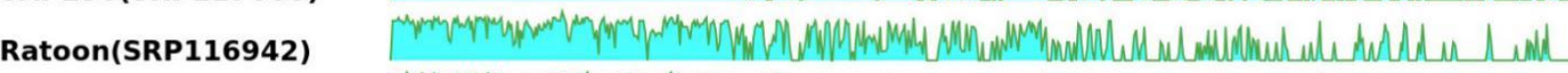

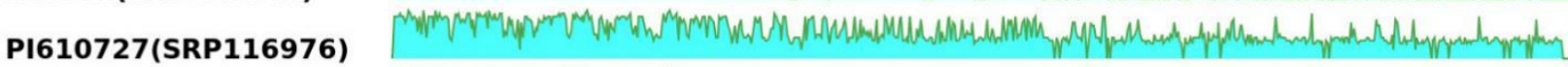
LiangTangAi(SRP015362)

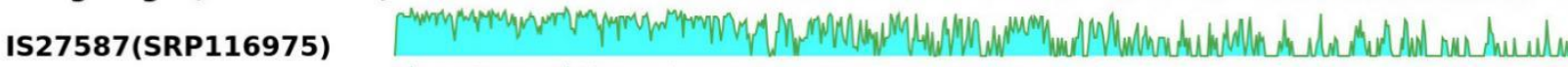

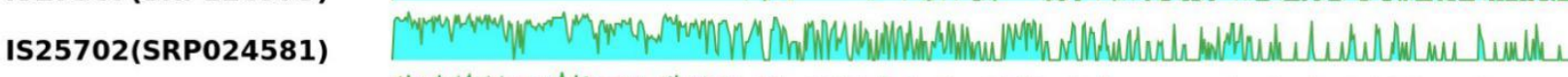

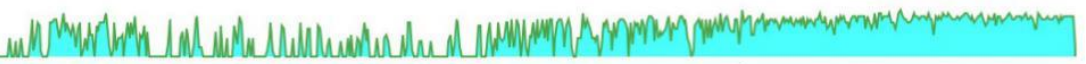

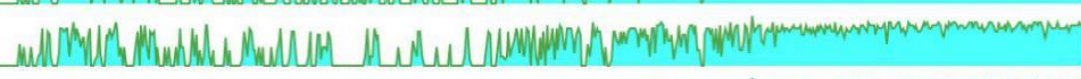

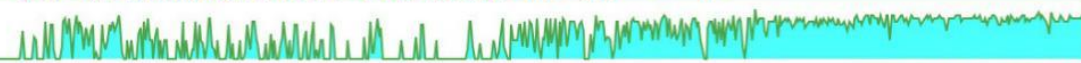

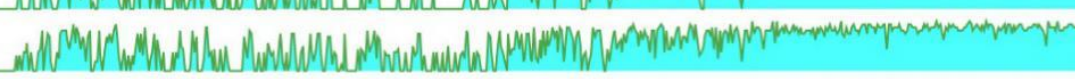

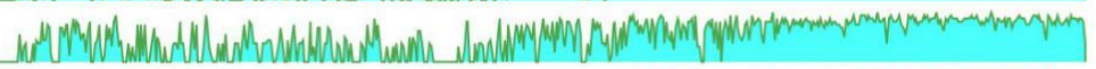

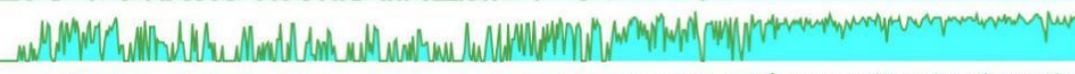

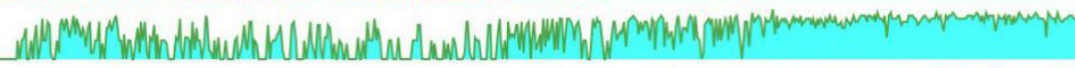

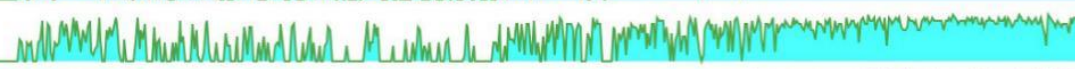

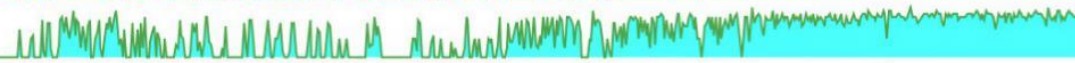

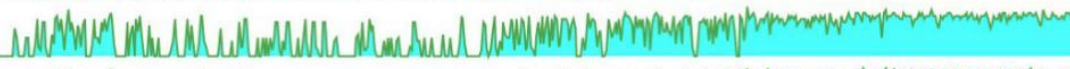

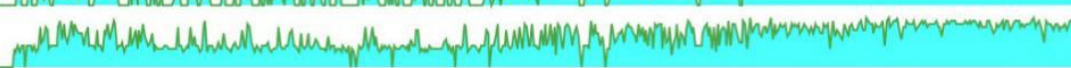

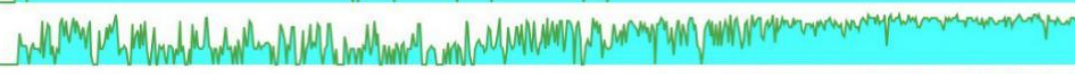

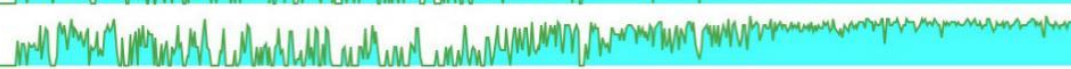

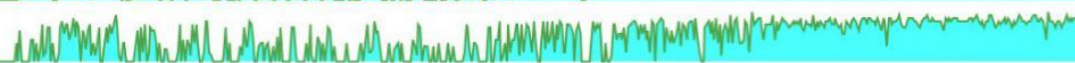

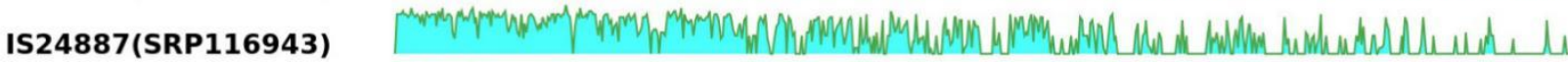

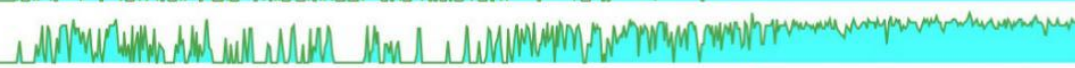

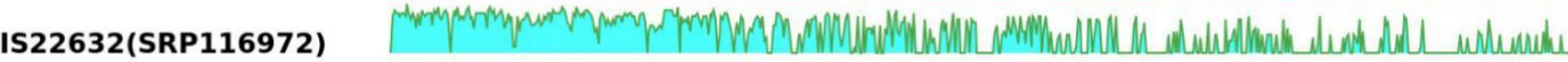

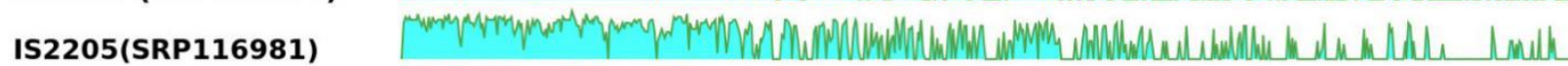

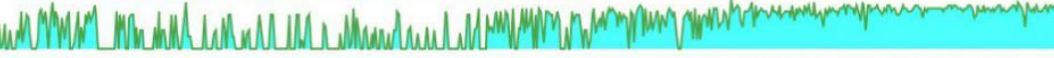

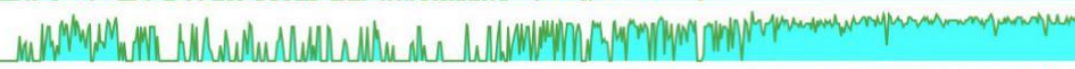

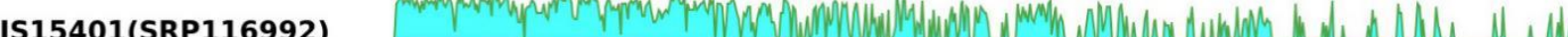

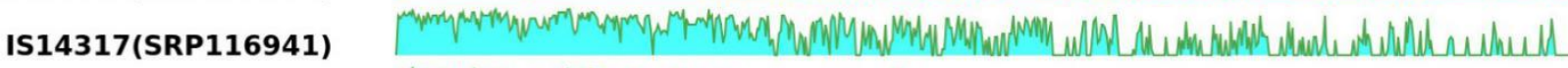
IS1054(M35-1)(SRP116995)

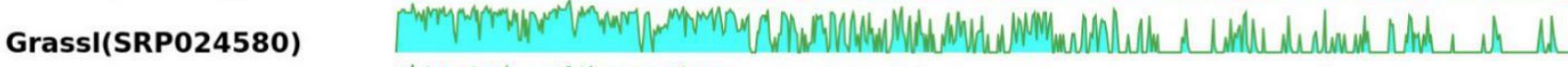

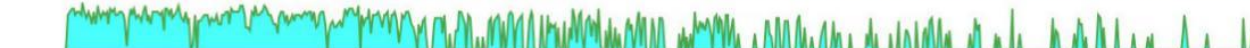

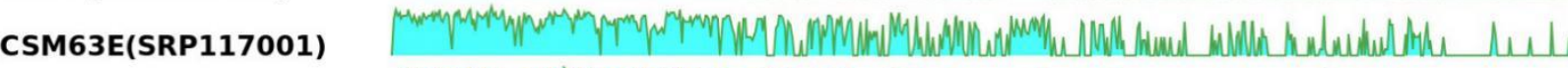

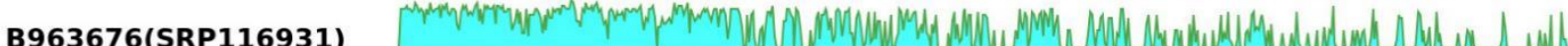
B35(SRP024579)

Chr07-genes

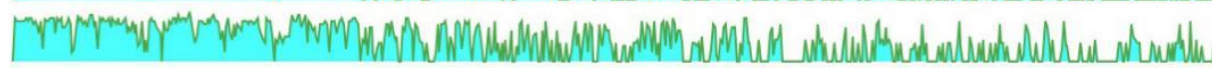

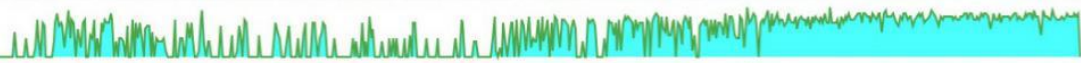

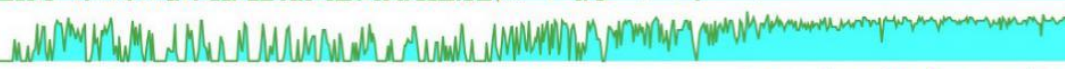

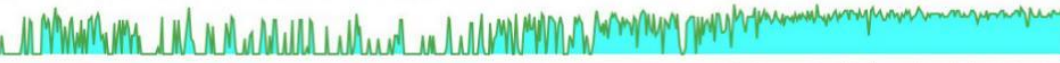

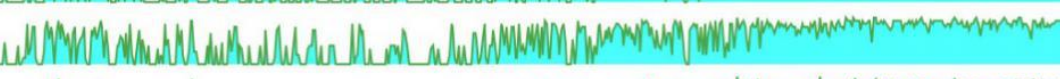

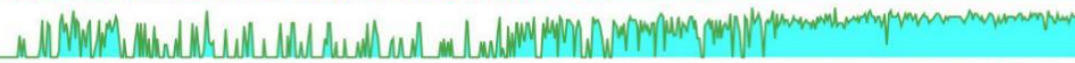

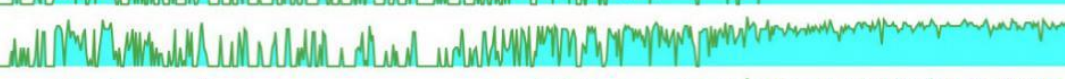

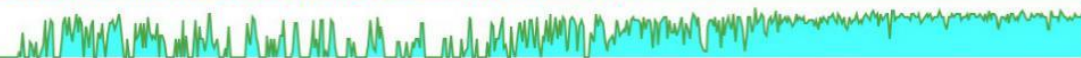

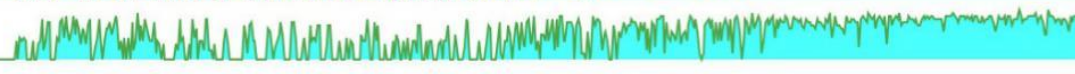

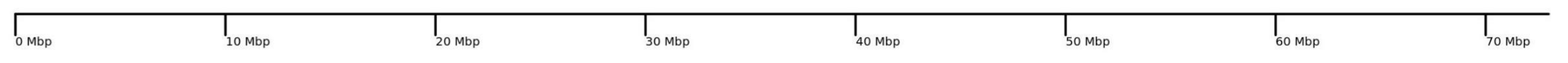




\section{Chromosome8}

V.virgatum(SRP116940)

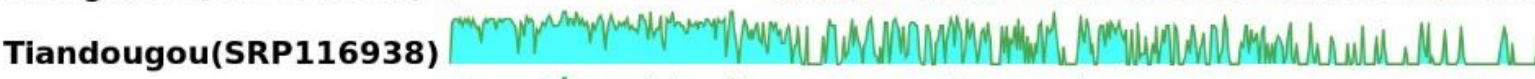
SSM29(SRP116939) SC650(SRP024584) SC566(SRP024585) SC52(SRP024582) SC301(SRP024578) SC110(SRP024583) SAP156(SRP117008) Ratoon(SRP116942) P1610727(SRP116976) LiangTangAi(SRP015362) IS27587(SRP116975) IS25702(SRP024581) IS24887(SRP116943) IS22632(SRP116972) IS2205(SRP116981) IS15401(SRP116992) IS14317(SRP116941) IS1054(M35-1)(SRP11699 GrassI(SRP024580) E36-1(SRP116991) CSM63E(SRP117001) B963676(SRP116931) B35(SRP024579) Chro8-genes 年

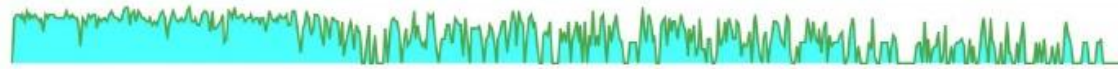

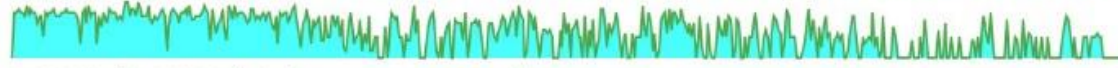

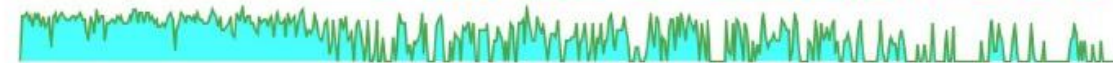

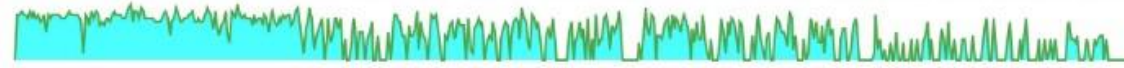

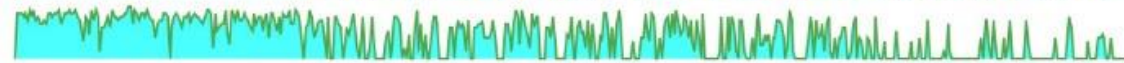

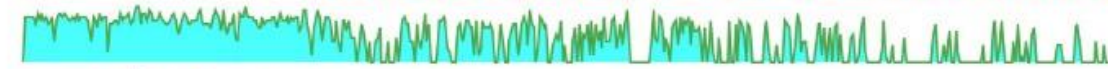

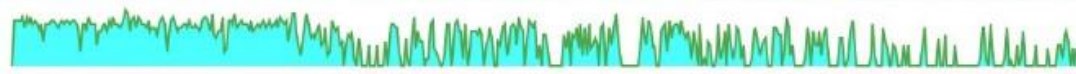

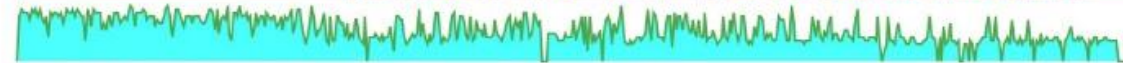

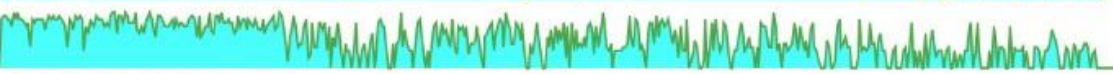

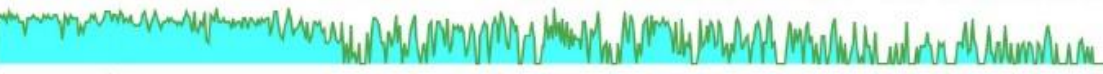

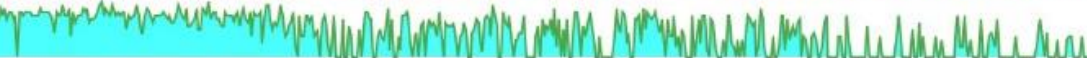

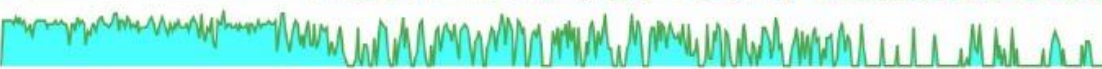

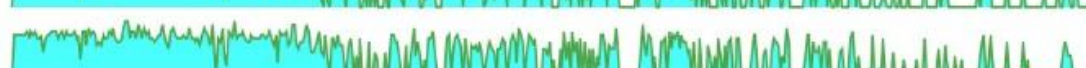
(a)

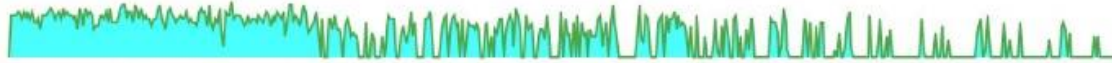

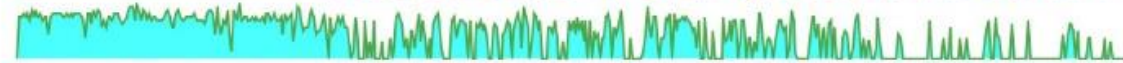

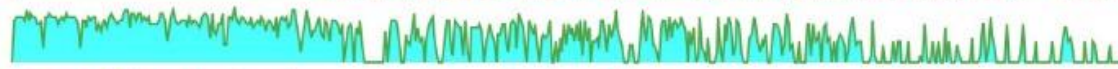

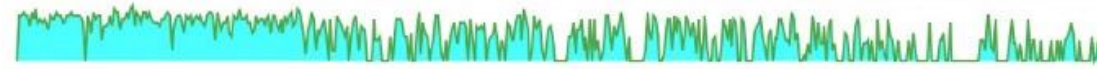

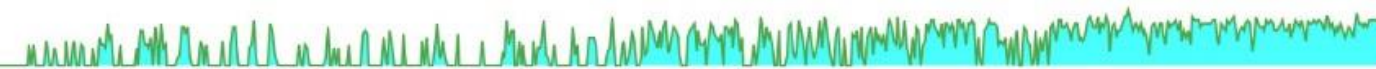

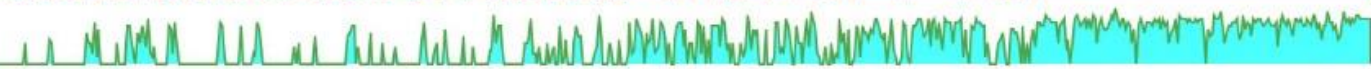

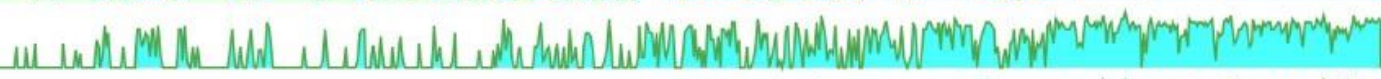

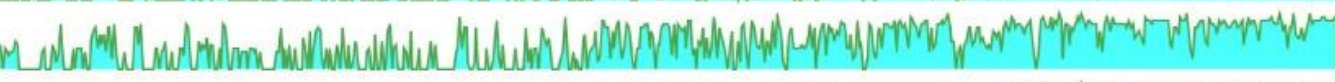

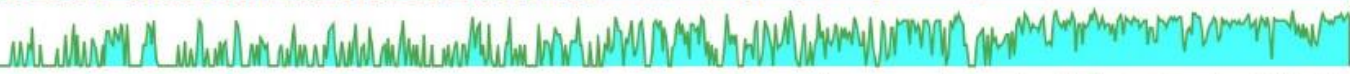

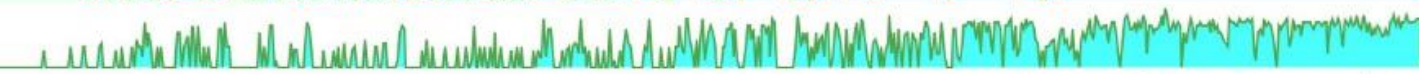

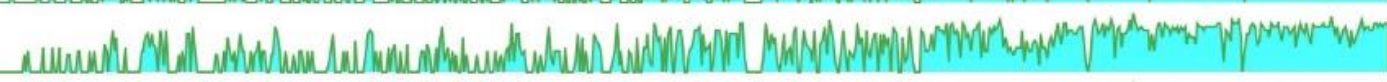

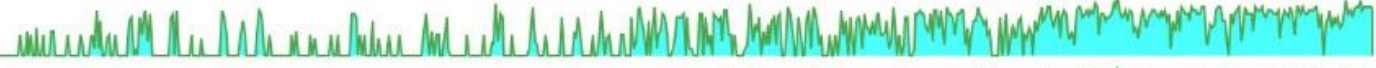

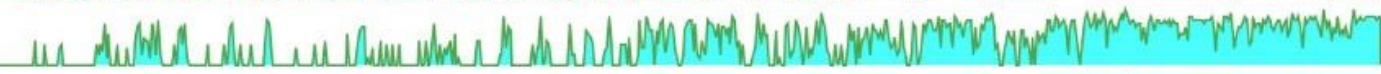

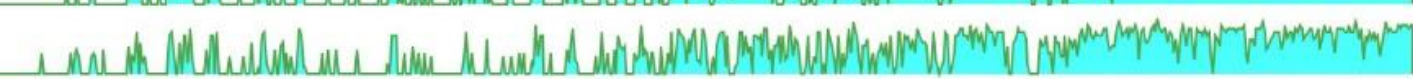

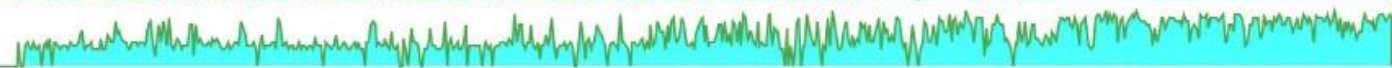

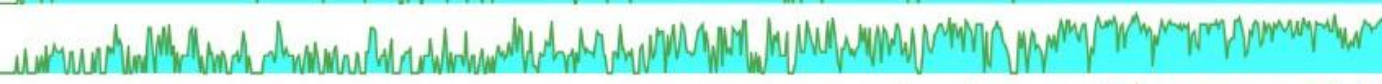

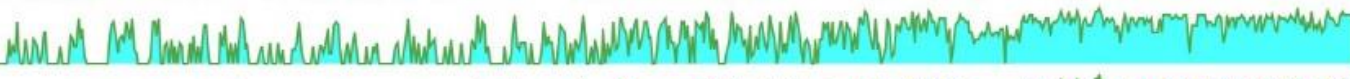

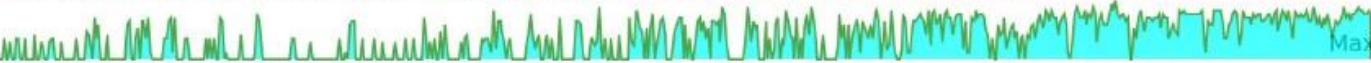

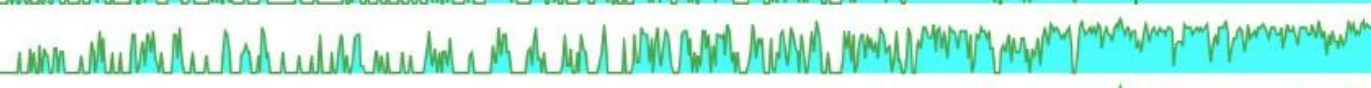

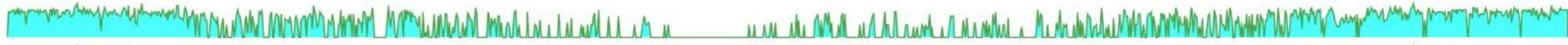

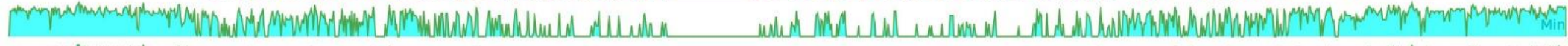

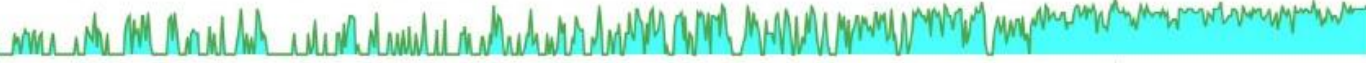

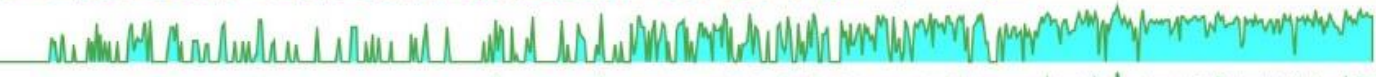

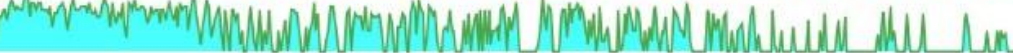

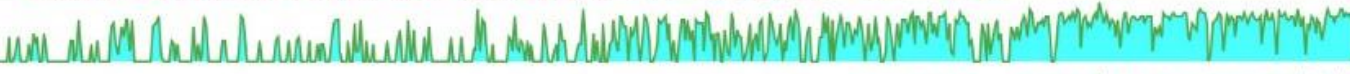

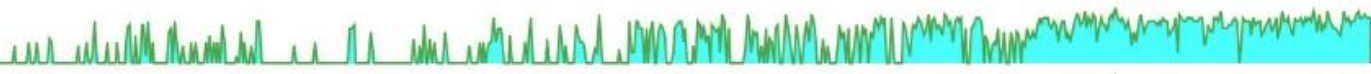

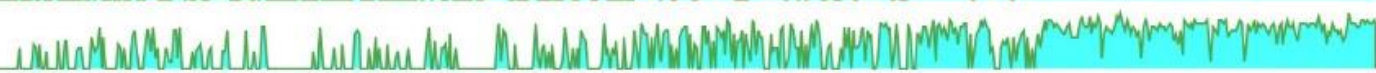

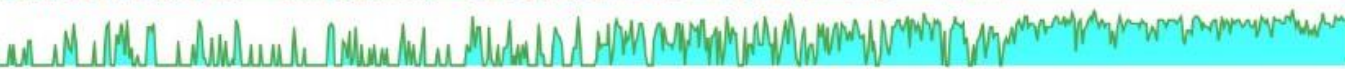

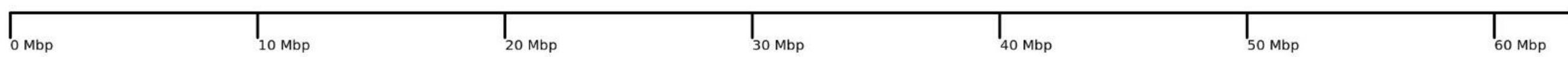




\section{Chromosome9}

V.virgatum(SRP116940)
Tiandougou(SRP116938) SSM29(SRP116939) SC650(SRP024584) SC566(SRP024585) SC52(SRP024582) SC301(SRP024578) SC110(SRP024583)

SAP156(SRP117008) Ratoon(SRP116942) P1610727(SRP116976) LiangTangAi(SRP015362) IS27587(SRP116975) IS25702(SRP024581) IS24887(SRP116943) IS22632(SRP116972) IS2205(SRP116981) IS15401(SRP116992) IS14317(SRP116941) IS1054(M35-1)(SRP116995) GrassI(SRP024580) E36-1(SRP116991) CSM63E(SRP117001) B963676(SRP116931) B35(SRP024579)

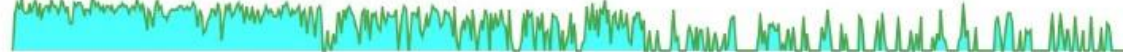

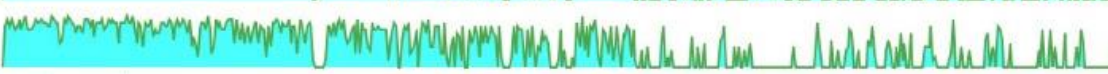

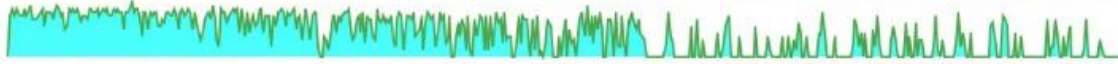

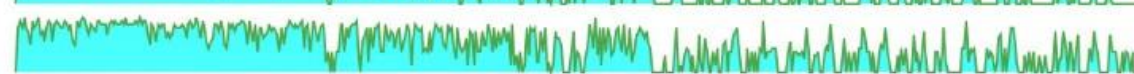

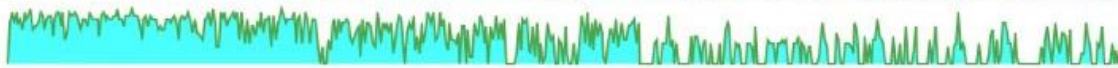

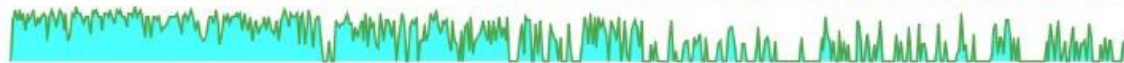

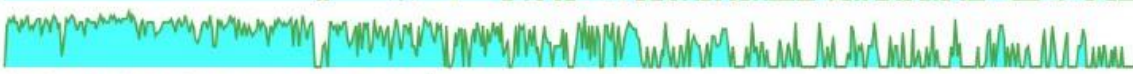

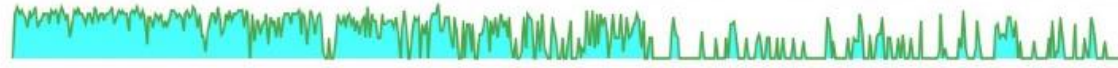

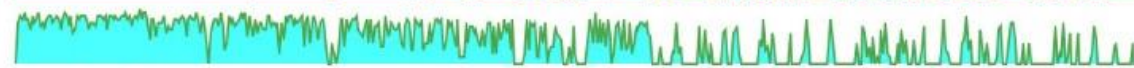

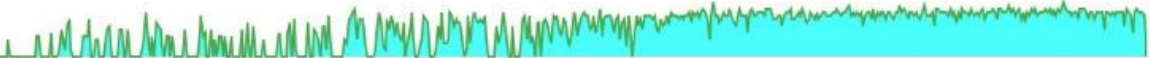

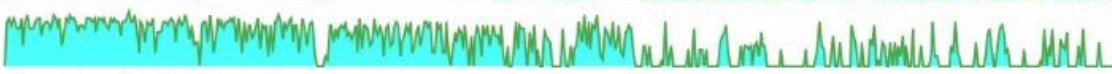

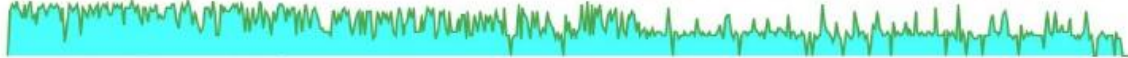

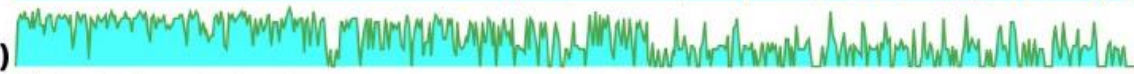

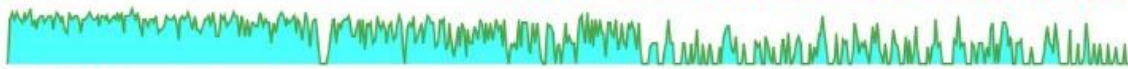

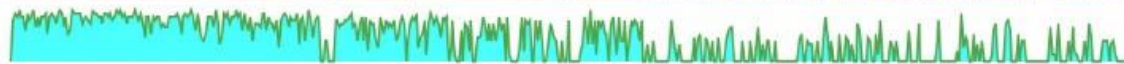

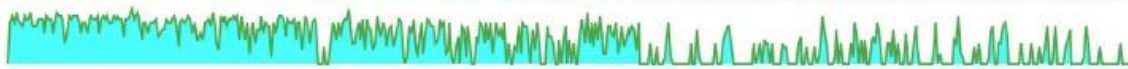

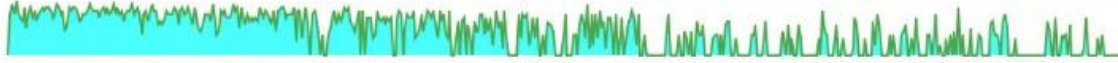

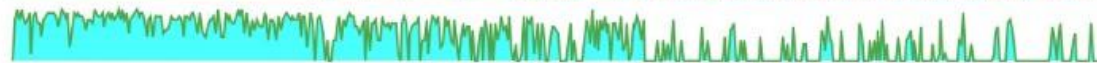

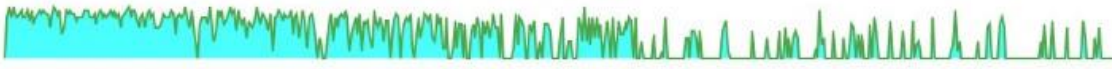

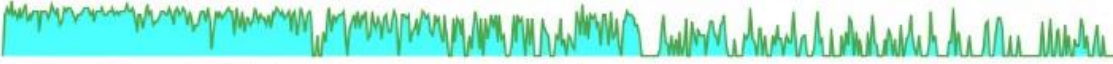
9.5\%)

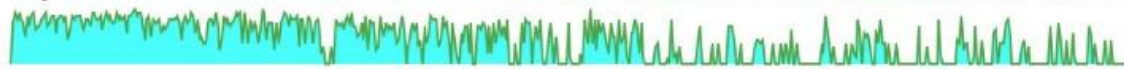

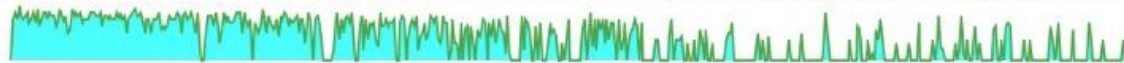

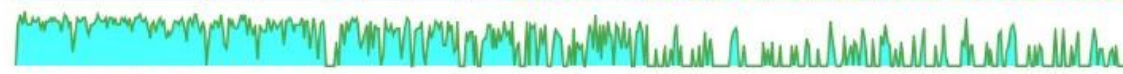

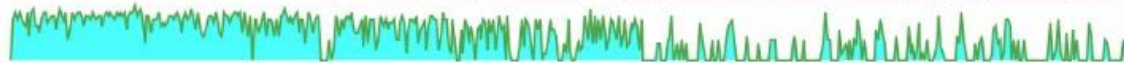

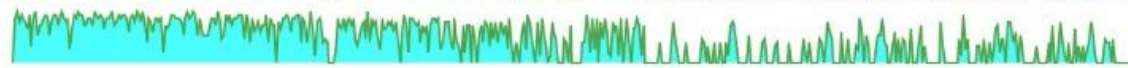

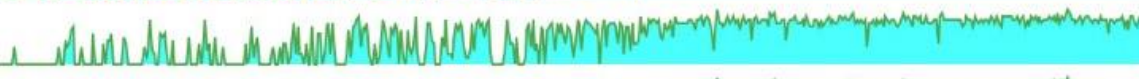

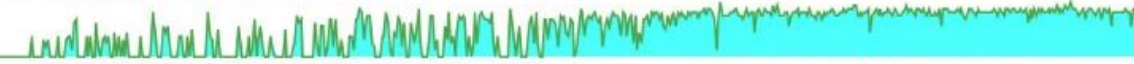

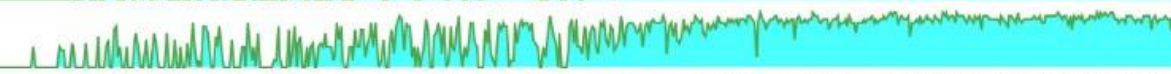

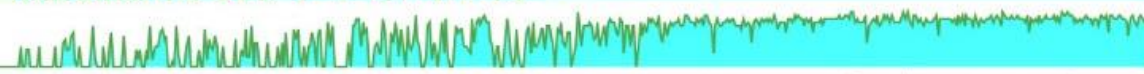

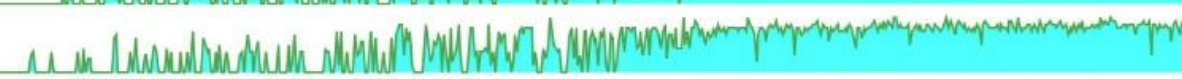

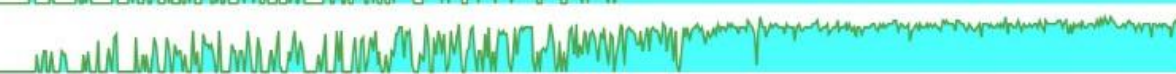

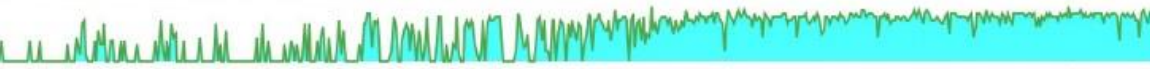

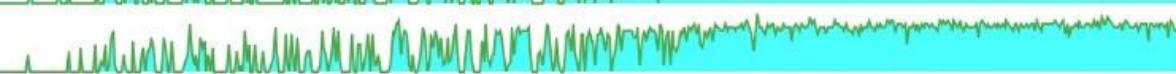

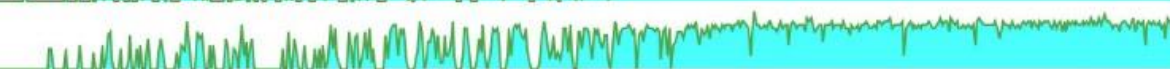

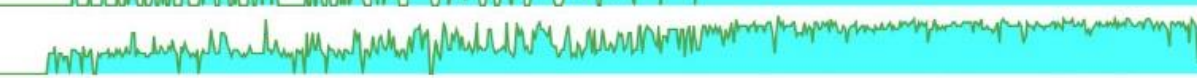

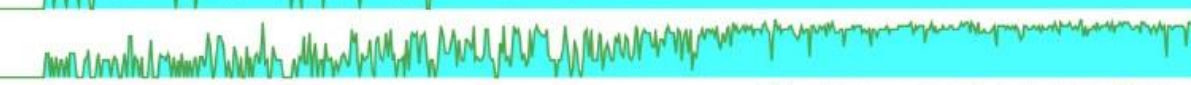

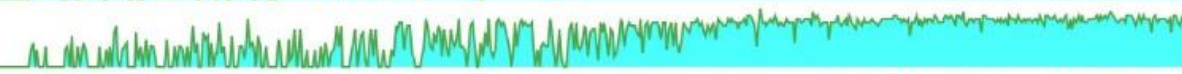

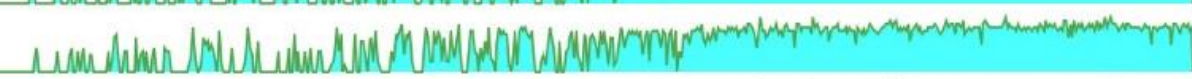

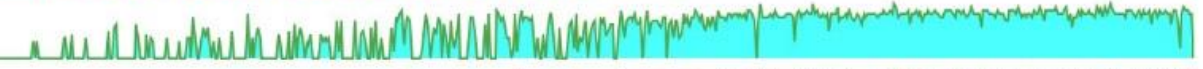

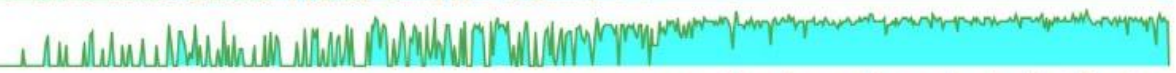

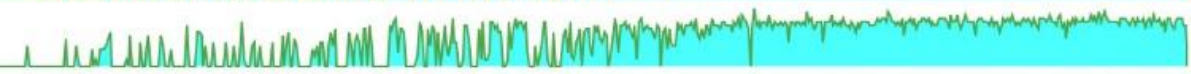

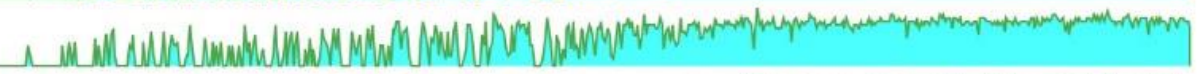

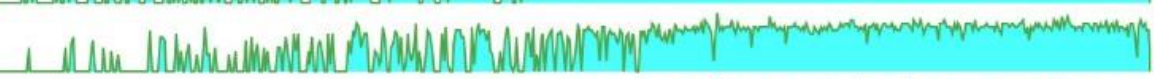

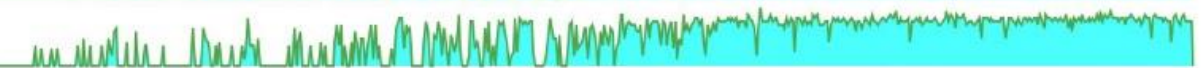

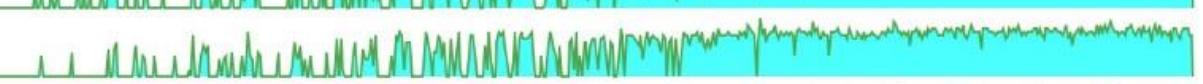

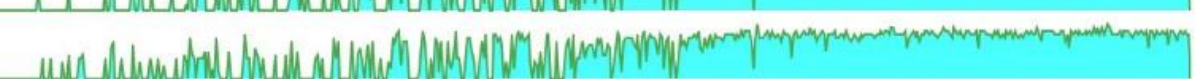

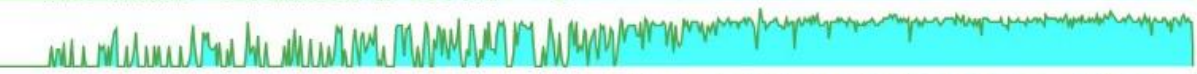

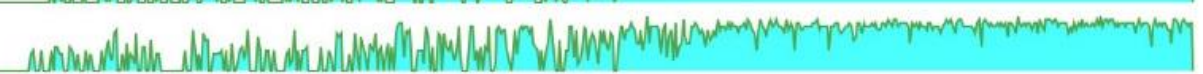

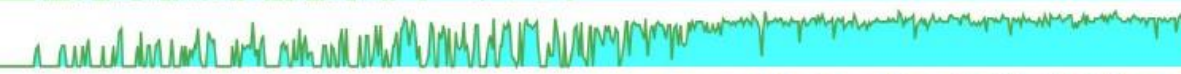

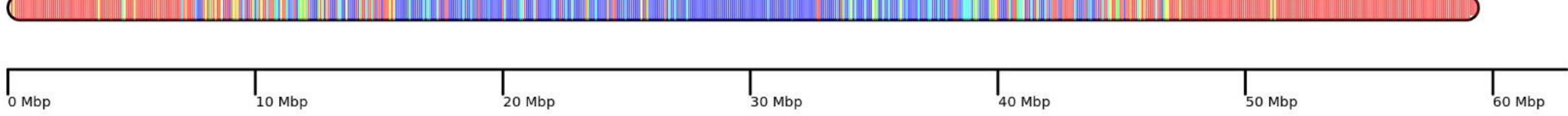




\section{Chromosome10}

V.virgatum(SRP116940) Tiandougou(SRP116938) SSM29(SRP116939) SC650(SRP024584) SC566(SRP024585) SC52(SRP024582) SC301(SRP024578) SC110(SRP024583) SAP156(SRP117008) Ratoon(SRP116942) P1610727(SRP116976) LiangTangAi(SRP015362) IS27587(SRP116975) IS25702(SRP024581) IS24887(SRP116943) IS22632(SRP116972) IS2205(SRP116981) IS15401(SRP116992) IS14317(SRP116941) IS1054(M35-1)(SRP116995) GrassI(SRP024580) E36-1(SRP116991) CSM63E(SRP117001) B963676(SRP116931) B35(SRP024579)

Chr10-genes

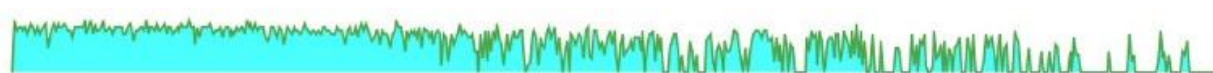

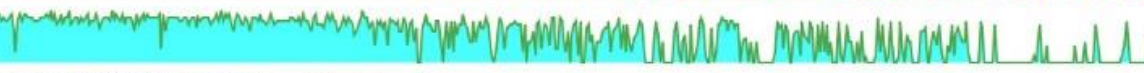

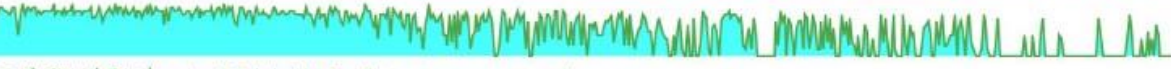

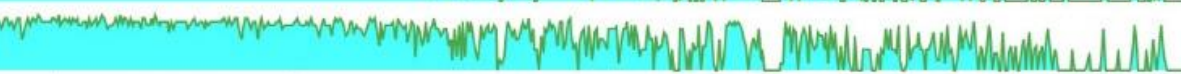

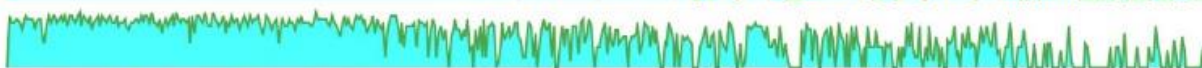

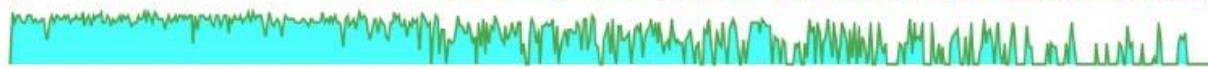

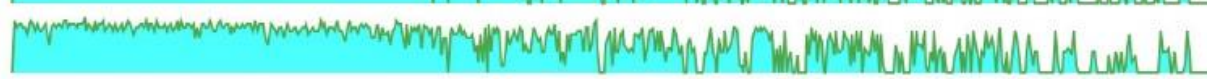

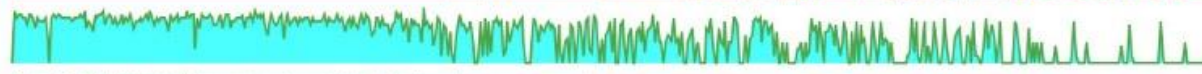

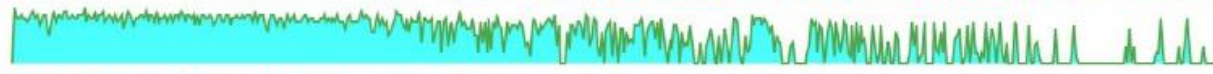

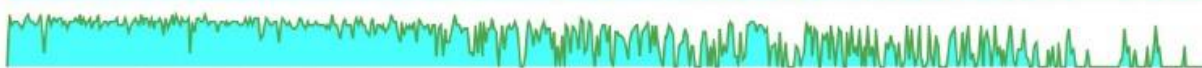

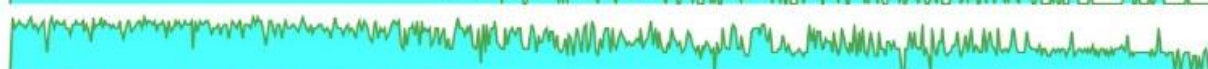

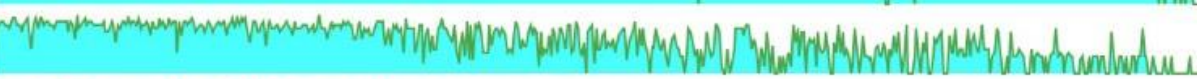

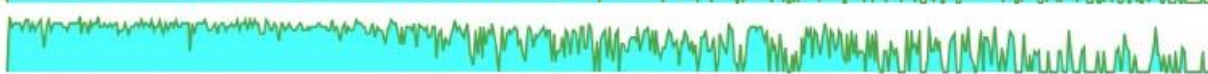

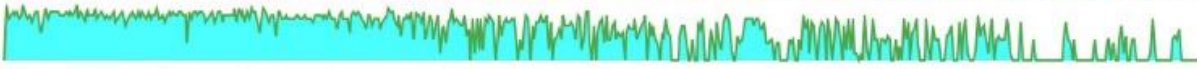

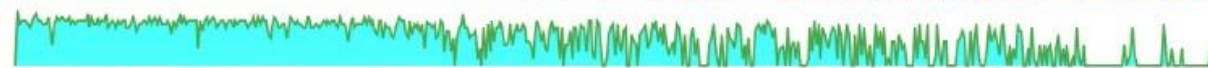

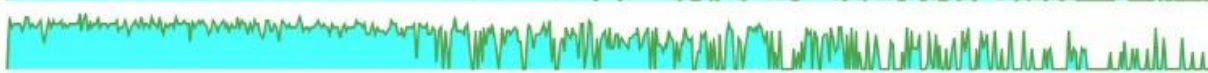

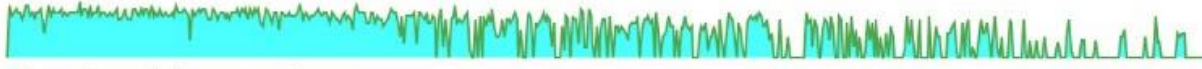

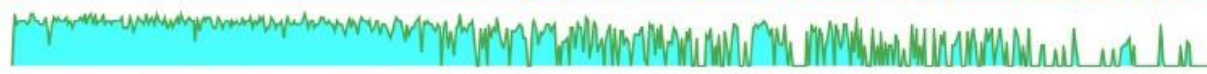

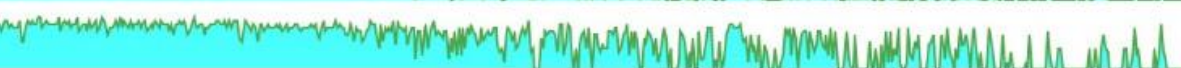

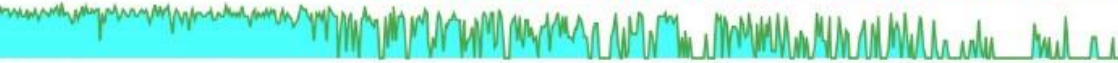

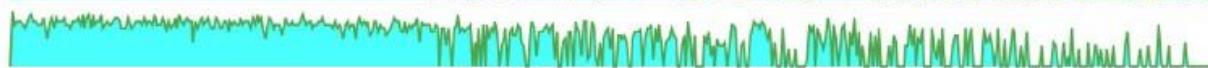

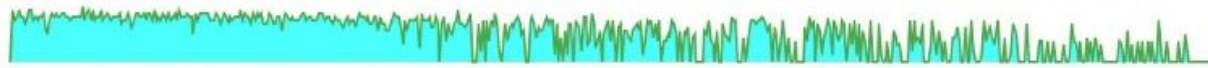

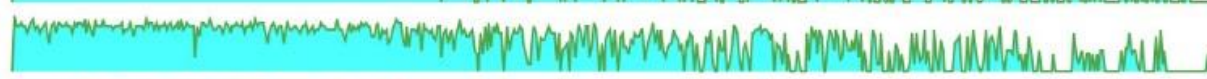

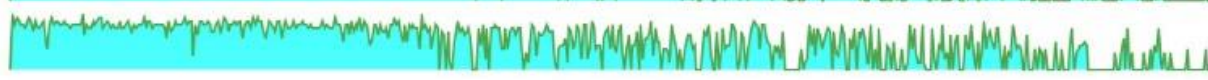

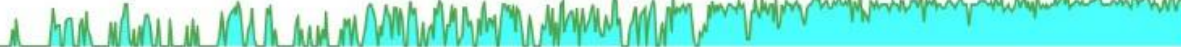

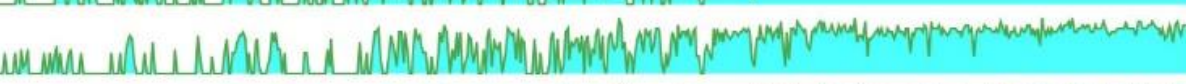

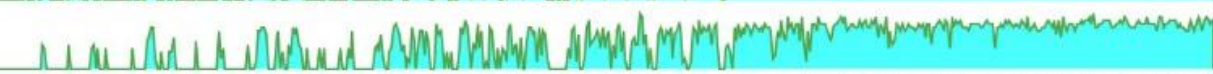

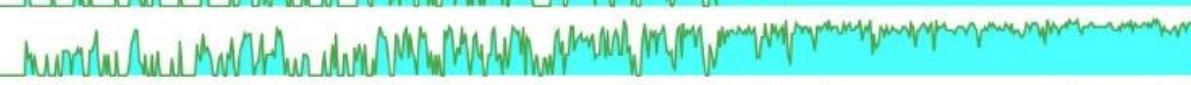

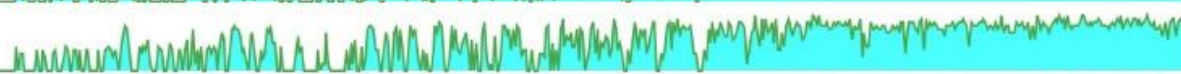

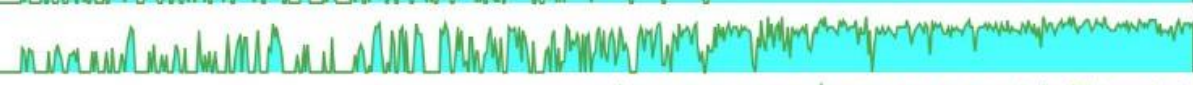

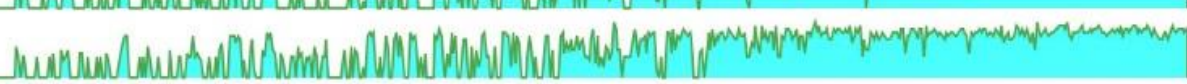

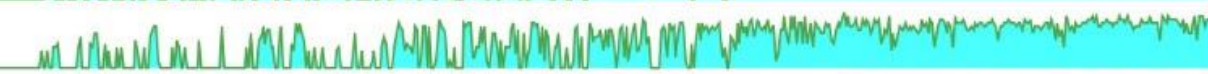

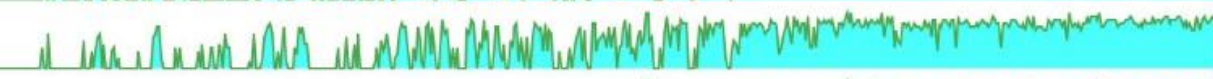

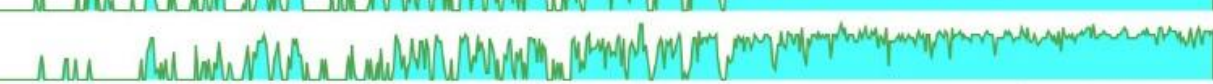

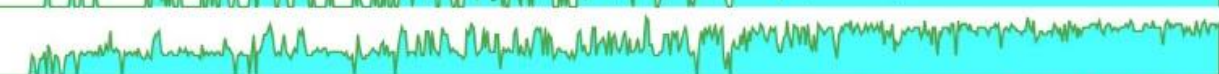

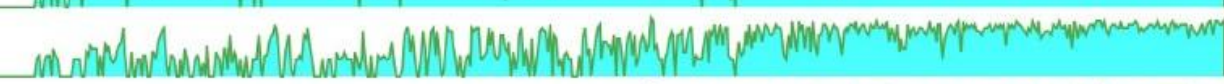

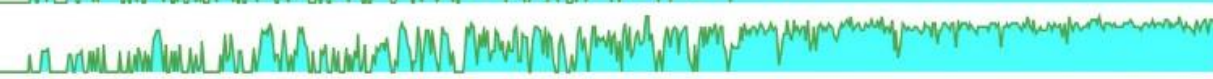

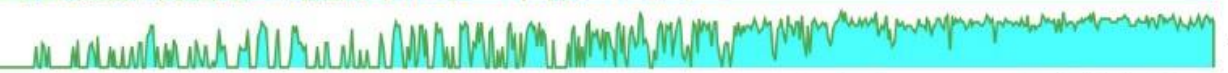

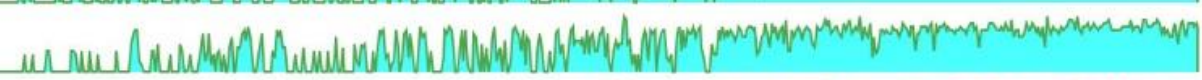

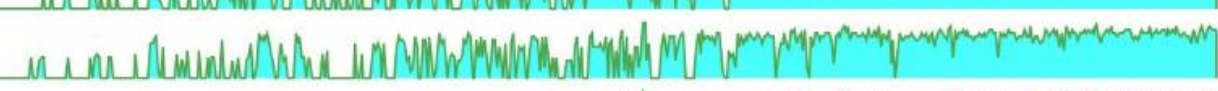

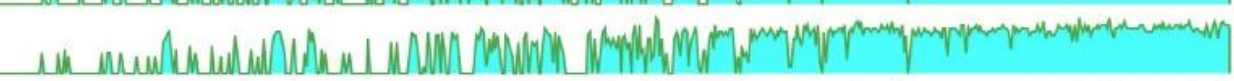

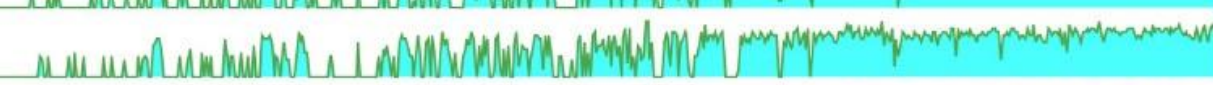

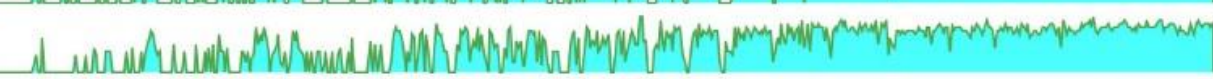

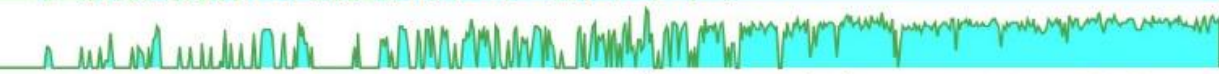

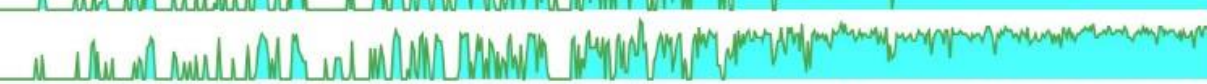

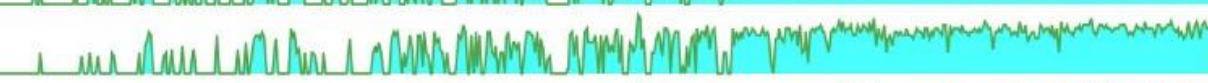

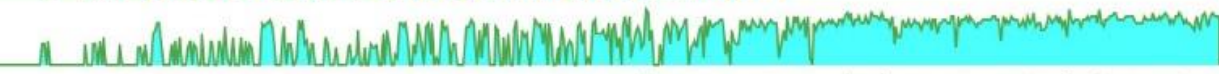

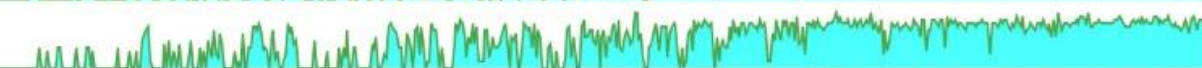

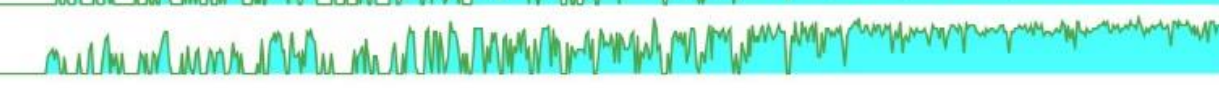

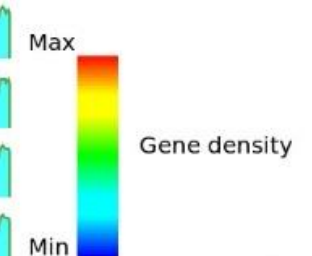

Gene density

$\Gamma_{\mathrm{Mbp}}$

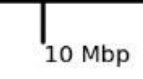

20 Mbp

$30 \mathrm{Mbp}$

$T_{40 \text { Mbp }}$

50 Mbp 
k.

\section{Scaffolds}

V.virgatum(SRP116940)

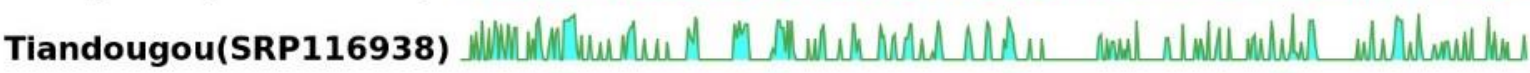

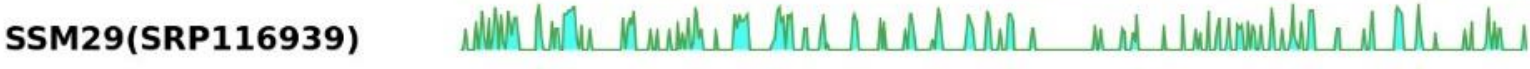

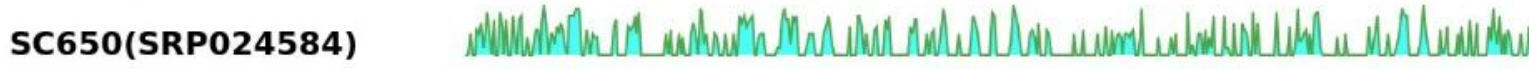

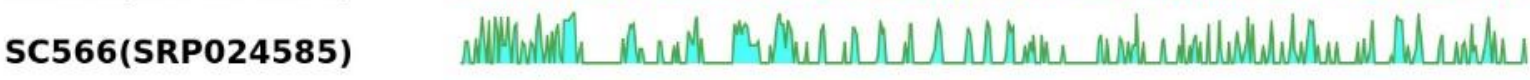

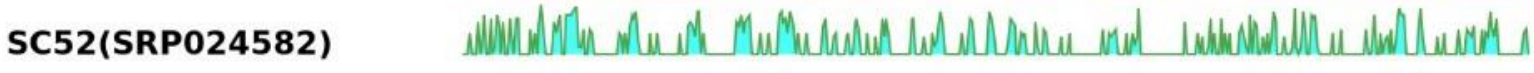

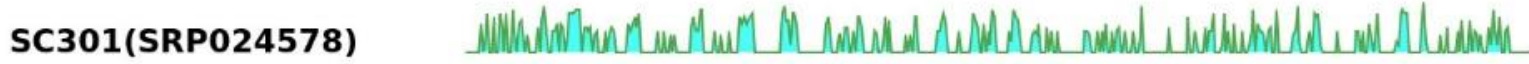

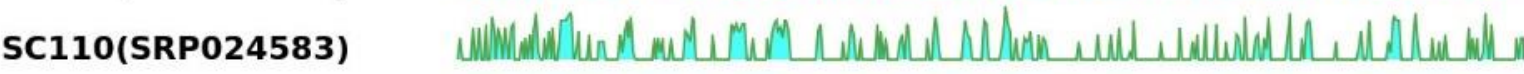

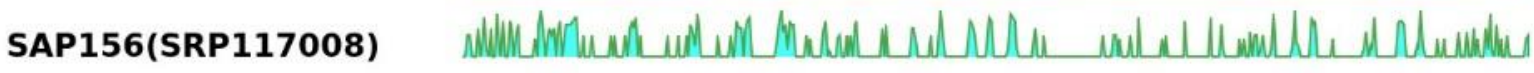

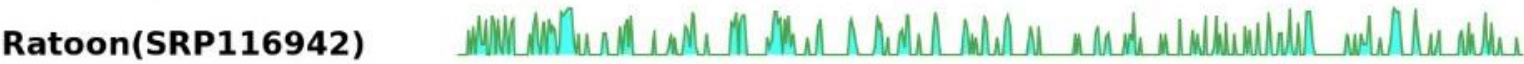

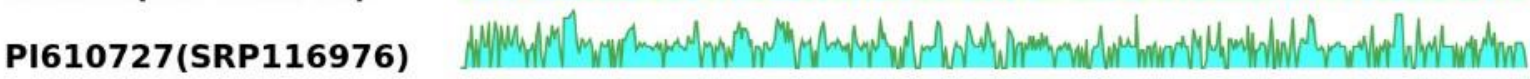

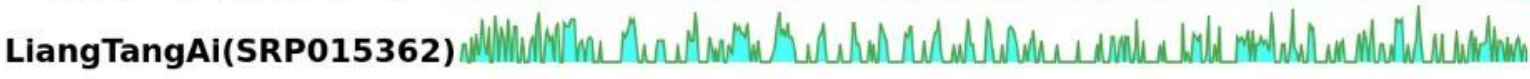

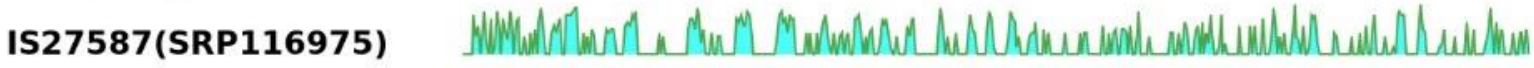

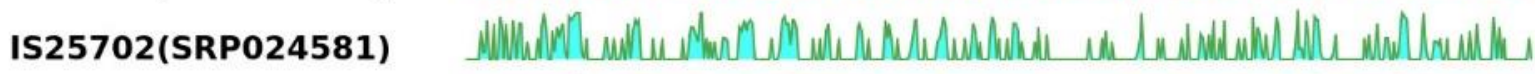

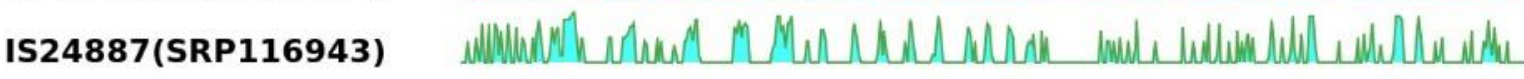

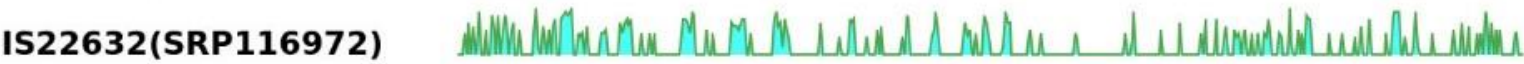

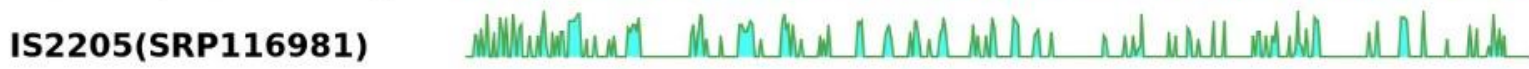

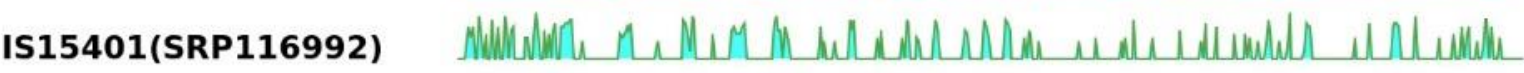

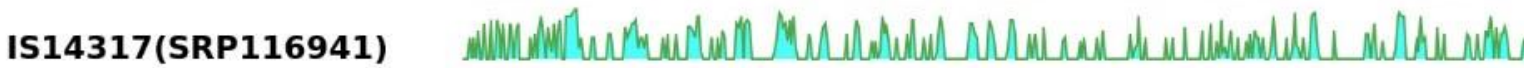
IS1054(M35-1)(SRP116995)

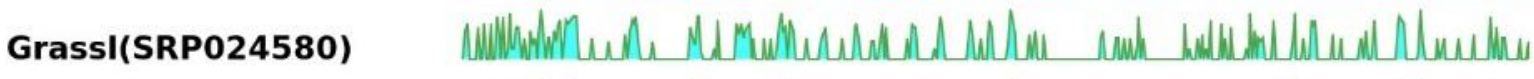

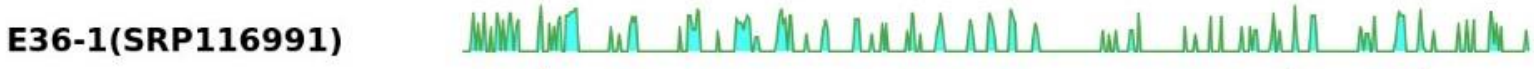

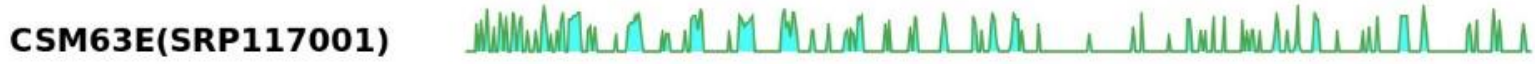

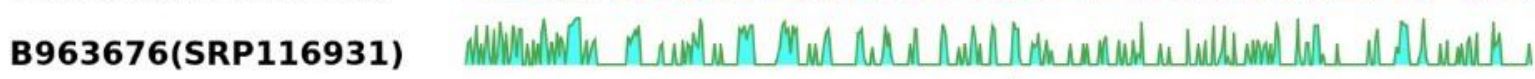
B35(SRP024579)

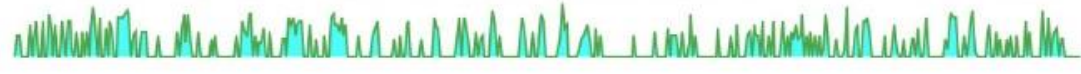

scaffolds-genes

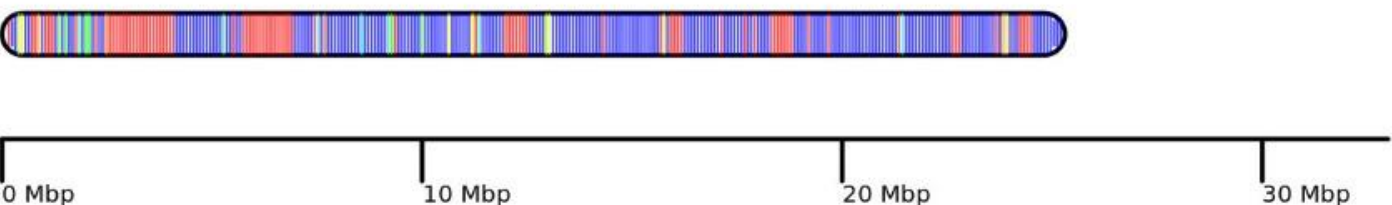




\section{ExtraContigs}

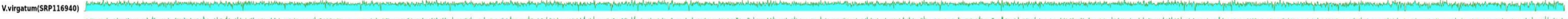
Wnovogow

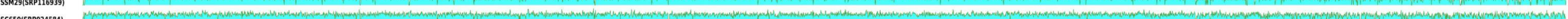

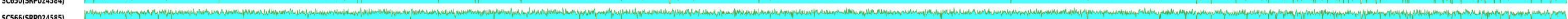

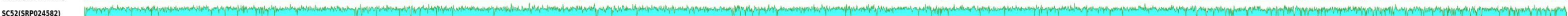

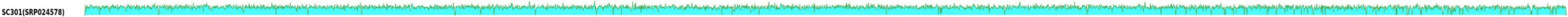

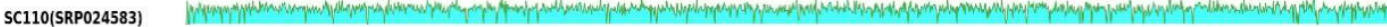

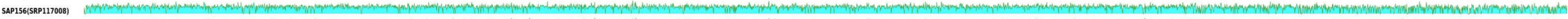

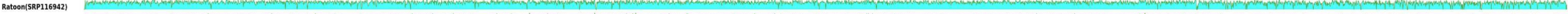

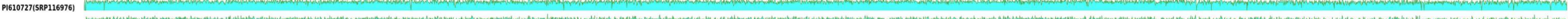

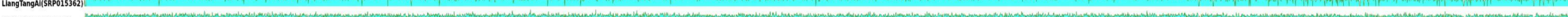

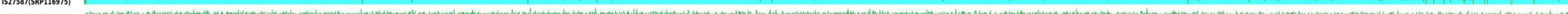

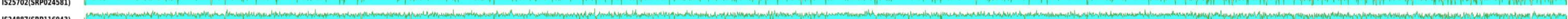

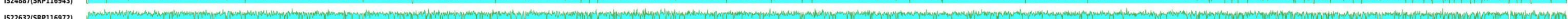

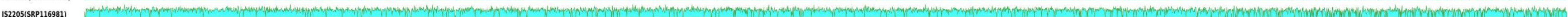

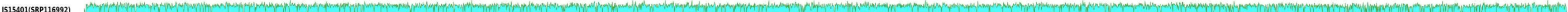

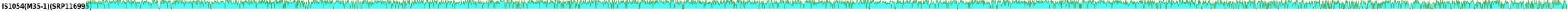

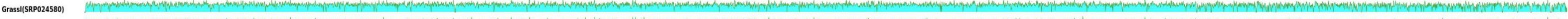

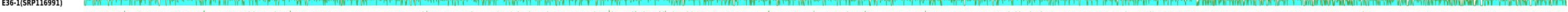

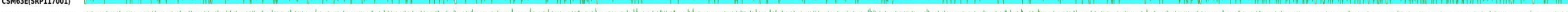

6

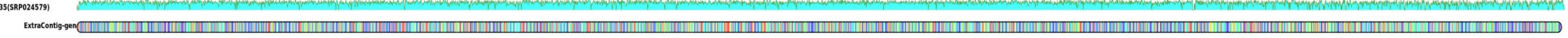
inotos $\mathbb{1}_{40 \mathrm{bep}}$ solese wowo lowe wowo 100 wep 10000 weo sonos sorose $\infty$ seores

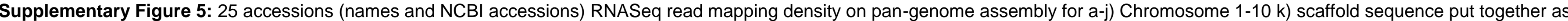
single sequence and I) non reference sequence assembly contigs from sorghum accessions concatenated to single sequence as extra contig sequence. 